\title{
Synthesis of Polycyclic Aromatic Iodides via ICl-Induced Intramolecular Cyclization
}

\author{
Tuanli Yao, Marino A. Campo and Richard C. Larock* \\ Department of Chemistry, Iowa State University, Ames, Iowa 50011
}

\section{Supporting Information}

General. ${ }^{1} \mathrm{H}$ and ${ }^{13} \mathrm{C}$ NMR spectra were recorded at 300 and $75.5 \mathrm{MHz}$. Thin-layer chromatography was performed using commercially prepared 60-mesh silica gel plates (Whatman K6F), and visualization was effected with short wavelength UV light (254 nm). All melting points are uncorrected. High resolution mass spectra were recorded on a Kratos MS50TC double focusing magnetic sector mass spectrometer using EI at $70 \mathrm{eV}$. All reagents were used directly as obtained commercially unless otherwise noted. 3-(2Iodophenyl)benzofuran, ${ }^{1}$ 2'-iodobiphenyl-4-carbaldehyde, ${ }^{2}$ 2-(2-iodophenyl)naphthalene, ${ }^{3}$ 2ethynylbiphenyl ${ }^{4}$ and 2-(phenylethynyl)phenylboronic acid $^{5}$ were prepared according to previous literature procedures.

General procedure for preparation of the 2-(arylethynyl)biphenyls. To a solution of the corresponding aryl iodide $(1.0 \mathrm{mmol})$ and the terminal alkyne $\left(1.2 \mathrm{mmol}, 1.2\right.$ equiv) in $\mathrm{Et}_{3} \mathrm{~N}$ (4 mL) were added $\mathrm{PdCl}_{2}\left(\mathrm{PPh}_{3}\right)_{2}(1.4 \mathrm{mg}, 2 \mathrm{~mol} \%)$ and $\mathrm{CuI}(2 \mathrm{mg}, 1 \mathrm{~mol} \%)$. The resulting mixture was then heated under an $\mathrm{N}_{2}$ atmosphere at $55^{\circ} \mathrm{C}$ for $3 \mathrm{~h}$. The mixture was allowed to cool to room temperature, and the ammonium salt was removed by filtration. The solvent was removed under reduced pressure and the residue was purified by column chromatography on silica gel to afford the corresponding product. 
2-[(4-Methoxyphenyl)ethynyl]biphenyl (1). 2-Ethynylbiphenyl and 4-iodoanisole were employed. Purification by flash chromatography (30:1 hexane/EtOAc) afforded $211 \mathrm{mg}$ (74\%) of the product as a clear liquid: ${ }^{1} \mathrm{H} \mathrm{NMR}\left(\mathrm{CDCl}_{3}\right) \delta 3.80(\mathrm{~s}, 3 \mathrm{H}), 6.84(\mathrm{dd}, J=2.4,6.9 \mathrm{~Hz}, 2 \mathrm{H})$, $7.30(\mathrm{dd}, J=2.1,6.9 \mathrm{~Hz}, 2 \mathrm{H}), 7.34-7.50(\mathrm{~m}, 6 \mathrm{H}), 7.64-7.73(\mathrm{~m}, 3 \mathrm{H}) ;{ }^{13} \mathrm{C} \mathrm{NMR}\left(\mathrm{CDCl}_{3}\right) \delta 55.5$, 88.4, 92.5, 114.2, 115.9, 122.2, 127.3, 127.6, 128.1, 128.4, 129.6, 129.7, 132.9, 133.1, 140.9, 143.9, 159.8; IR (neat, $\mathrm{cm}^{-1}$ ) 3059, 3017, 2214, 1605; HRMS Calcd for $\mathrm{C}_{21} \mathrm{H}_{16} \mathrm{O}: 284.1201$. Found: 284.1205 .

2-[(4-Methylphenyl)ethynyl]biphenyl (3). 2-Ethynylbiphenyl and 4-iodotoluene were employed. Purification by flash chromatography (40:1 hexane/EtOAc) afforded $204 \mathrm{mg}$ (76\%) of the product as a clear liquid: ${ }^{1} \mathrm{H}$ NMR $\left(\mathrm{CDCl}_{3}\right) \delta 2.35(\mathrm{~s}, 3 \mathrm{H}), 7.11(\mathrm{~d}, J=7.8 \mathrm{~Hz}, 2 \mathrm{H}), 7.24$ (d, $J=8.4 \mathrm{~Hz}, 2 \mathrm{H}), 7.31-7.50(\mathrm{~m}, 6 \mathrm{H}), 7.63-7.71(\mathrm{~m}, 3 \mathrm{H}) ;{ }^{13} \mathrm{C} \mathrm{NMR}\left(\mathrm{CDCl}_{3}\right) \delta$ 21.7, 89.0, 92.7, 120.6, 122.0, 127.3, 127.7, 128.1, 128.6, 129.3, 129.6, 129.7, 131.5, 133.0, 138.5, 140.9, 144.0; IR (neat, $\mathrm{cm}^{-1}$ ) 3058, 3025, 2919, 2215; HRMS Calcd for $\mathrm{C}_{21} \mathrm{H}_{16}$ : 268.1252. Found: 268.1257. 2-(Phenylethynyl)biphenyl (5). 2-Iodobiphenyl and phenylacetylene were employed. Purification by flash chromatography (30:1 hexane/EtOAc) afforded $254 \mathrm{mg}(100 \%)$ of the product as a clear liquid with spectral properties identical to those previously reported. ${ }^{7}$

Ethyl 4-(biphen-2-ylethynyl)benzoate (7). 2-Ethynylbiphenyl and ethyl 4-iodobenzoate were employed. Purification by flash chromatography (15:1 hexane/EtOAc) afforded $274 \mathrm{mg}$ (84\%) of the product as a white solid: $\mathrm{mp} 58-60{ }^{\circ} \mathrm{C}$; ${ }^{1} \mathrm{H} \mathrm{NMR}\left(\mathrm{CDCl}_{3}\right) \delta 1.38(\mathrm{t}, J=6.9 \mathrm{~Hz}$, $3 \mathrm{H}), 4.36(\mathrm{q}, J=7.2 \mathrm{~Hz}, 2 \mathrm{H}), 7.32-7.48(\mathrm{~m}, 8 \mathrm{H}), 7.64-7.66(\mathrm{~m}, 3 \mathrm{H}), 7.96(\mathrm{~d}, J=8.4 \mathrm{~Hz}, 2 \mathrm{H}) ;{ }^{13} \mathrm{C}$ $\operatorname{NMR}\left(\mathrm{CDCl}_{3}\right) \delta$ 14.6, 61.3, 91.7, 92.6, 121.3, 127.4, 127.9, 128.2, 128.3, 129.3, 129.63, 129.64, 129.8, 129.9, 131.4, 133.2, 140.6, 144.5, 166.3; IR (neat, $\mathrm{cm}^{-1}$ ) 3263, 1718; HRMS Calcd for $\mathrm{C}_{23} \mathrm{H}_{18} \mathrm{O}_{2}$ : 326.1307. Found: 326.1312 . 
2-(4-Nitrophenylethynyl)biphenyl (9). 2-Ethynylbiphenyl and 1-iodo-4-nitrobenzene were employed. Purification by flash chromatography (15:1 hexane/EtOAc) afforded $269 \mathrm{mg}$ (90\%) of the product as a yellow solid: $\mathrm{mp} 100-101{ }^{\circ} \mathrm{C} ;{ }^{1} \mathrm{H} \mathrm{NMR}\left(\mathrm{CDCl}_{3}\right) \delta 7.38-7.50(\mathrm{~m}, 8 \mathrm{H})$, 7.62-7.68 (m, 3H), $8.16(\mathrm{~d}, J=7.6 \mathrm{~Hz}, 2 \mathrm{H}) ;{ }^{13} \mathrm{C} \mathrm{NMR}\left(\mathrm{CDCl}_{3}\right) \delta$ 90.6, 95.2, 120.7, 123.8, 127.5, 128.0, 128.2, 129.6, 129.8, 129.9, 130.6, 132.2, 133.3, 140.5, 144.8; IR (neat, $\left.\mathrm{cm}^{-1}\right) 3062,2215$, 1593, 1516; HRMS Calcd for $\mathrm{C}_{20} \mathrm{H}_{12} \mathrm{NO}_{2}$ : 299.0946. Found: 299.0950 .

2'-(Phenylethynyl)biphenyl-4-carbaldehyde (11). 2'-Iodobiphenyl-4-carbaldehyde and phenylacetylene were employed. Purification by flash chromatography (4:1 hexane/EtOAc) afforded $273 \mathrm{mg}(97 \%)$ of the product as a white solid: $\mathrm{mp} 90-93{ }^{\circ} \mathrm{C} ;{ }^{1} \mathrm{H} \mathrm{NMR}\left(\mathrm{CDCl}_{3}\right) \delta 7.27-$ $7.32(\mathrm{~m}, 5 \mathrm{H}), 7.38-7.43(\mathrm{~m}, 3 \mathrm{H}), 7.66-7.68(\mathrm{~m}, 1 \mathrm{H}), 7.83(\mathrm{~d}, J=6.0 \mathrm{~Hz}, 2 \mathrm{H}), 7.96(\mathrm{~d}, J=6.3 \mathrm{~Hz}$, 2H), $10.08(\mathrm{~s}, 1 \mathrm{H}) ;{ }^{13} \mathrm{C} \mathrm{NMR}\left(\mathrm{CDCl}_{3}\right) \delta 88.7,92.9,121.7,123.1,128.1,128.4,128.5,128.8$, 129.4, 130.2, 131.4, 133.2, 135.4, 142.4, 146.9, 192.2 (missing one $\mathrm{sp}^{2}$ carbon due to overlap); IR (neat, $\mathrm{cm}^{-1}$ ) 3058, 1701, 1605; HRMS Calcd for $\mathrm{C}_{21} \mathrm{H}_{14} \mathrm{O}$ : 282.1045. Found: 282.1049.

3-[2-(Phenylethynyl)phenyl]benzofuran (15). 3-(2-Iodophenyl)benzofuran ${ }^{1}$ and phenylacetylene were employed. Purification by flash chromatography (40:1 hexane/EtOAc) afforded $253 \mathrm{mg}(86 \%)$ of the product as a light yellow liquid: ${ }^{1} \mathrm{H} \mathrm{NMR}\left(\mathrm{CDCl}_{3}\right) \delta$ 7.22-7.45 (m,

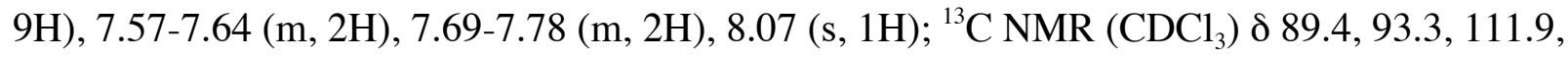
$120.7,121.3,122.5,123.0,123.4,124.6,127.3,127.6,128.5,128.8,129.6,131.6,133.4,134.0$, 135.4, 143.8, 155.5; IR (neat, $\mathrm{cm}^{-1}$ ) 3058, 1601; HRMS Calcd for $\mathrm{C}_{22} \mathrm{H}_{14} \mathrm{O}: 294.1045$. Found: 294.1047.

2-(Biphen-2-ylethynyl)thiophene (17). 2-Ethynylbiphenyl and 2-iodothiophene were employed. Purification by flash chromatography (15:1 hexane/EtOAc) afforded $221 \mathrm{mg}(85 \%)$ of the product as a light yellow liquid: ${ }^{1} \mathrm{H}$ NMR $\left(\mathrm{CDCl}_{3}\right) \delta$ 6.98-7.00 (m, 1H), 7.15-7.17 (m, 
1H), 7.25-7.27 (m, 1H), 7.37-7.55 (m, 6H), 7.66-7.75 (m, 3H); ${ }^{13} \mathrm{C} \mathrm{NMR}\left(\mathrm{CDCl}_{3}\right)$ 8 85.9, 93.5, 121.6, 123.8, 127.3, 127.4, 127.5, 127.8, 128.3, 129.0, 129.6, 129.8, 131.8, 132.8, 140.7, 144.0; IR (neat, $\mathrm{cm}^{-1}$ ) 3063, 2204, 1478; HRMS Calcd for $\mathrm{C}_{18} \mathrm{H}_{12} \mathrm{~S}: 260.0660$. Found: 260.0663 .

4-Phenyl-3-(phenylethynyl)isocoumarin (19). 3-Iodo-4-phenylisocoumarin was prepared by the following procedure. To a solution of 4-phenyl-3-(trimethylsilyl)isocoumarin ${ }^{6}(0.441 \mathrm{~g}$, $1.5 \mathrm{mmol})$ and $\mathrm{I}_{2}(1.14 \mathrm{~g}, 4.5 \mathrm{mmol})$ in $\mathrm{CH}_{3} \mathrm{CN}(15 \mathrm{~mL})$ under $\mathrm{N}_{2}$, was added $\mathrm{AgOTf}(0.78 \mathrm{~g}, 3.0$ mmol) in $\mathrm{CH}_{3} \mathrm{CN}(5 \mathrm{~mL})$ at room temperature. The reaction mixture was stirred at $55^{\circ} \mathrm{C}$ for 5 days. The mixture was allowed to cool to room temperature, diluted with diethyl ether (100 mL) and filtered. The filtrate was washed with satd aq $\mathrm{Na}_{2} \mathrm{~S}_{2} \mathrm{O}_{3}(25 \mathrm{~mL})$ and the organic layer dried $\left(\mathrm{MgSO}_{4}\right)$, and filtered. The solvent was evaporated under reduced pressure and the product was purified by chromatography on a silica gel column to afford $501 \mathrm{mg}(96 \%)$ of the product as a white solid: $\mathrm{mp} 170-17{ }^{\circ} \mathrm{C} ;{ }^{1} \mathrm{H}$ NMR $\left(\mathrm{CDCl}_{3}\right) \delta 6.98(\mathrm{~d}, J=12.0 \mathrm{~Hz}, 1 \mathrm{H}), 7.27(\mathrm{dd}, J=2.0$, 7.6 Hz, 2H), 7.50-7.63 (m, 5H), $8.31(\mathrm{dd}, J=0.8,8.0 \mathrm{~Hz}, 1 \mathrm{H}) ;{ }^{13} \mathrm{C} \mathrm{NMR}\left(\mathrm{CDCl}_{3}\right) \delta$ 107.9, 119.6, 125.6, 127.4, 128.7, 128.9, 129.1, 129.9, 130.5, 135.2, 137.0, 137.3, 161.2; IR (neat, $\left.\mathrm{cm}^{-1}\right) 1736$; HRMS Calcd for $\mathrm{C}_{15} \mathrm{H}_{9} \mathrm{O}_{2} \mathrm{I}$ : 347.9647 . Found: 347.9652.

3-Iodo-4-phenylisocoumarin and phenylacetylene were employed in the above Sonogashira coupling reaction. Purification by flash chromatography (7:1 hexane/EtOAc) afforded $148 \mathrm{mg}(46 \%)$ of the desired product 19 as a light yellow solid: $\mathrm{mp} 164-166{ }^{\circ} \mathrm{C} ;{ }^{1} \mathrm{H}$ NMR $\left(\mathrm{CDCl}_{3}\right) \delta$ 7.19-7.32 (m, 6H), 7.46-7.58 (m, 6H), 7.63-7.69 (m, 1H), $8.38(\mathrm{~d}, J=6.9 \mathrm{~Hz}$, $1 \mathrm{H}) ;{ }^{13} \mathrm{C} \mathrm{NMR}\left(\mathrm{CDCl}_{3}\right) \delta 82.5,96.9,121.5,121.6,124.3,125.7,128.6,128.83,128.84,129.1$, 129.6, 130.2, 130.9, 131.8, 133.6, 135.0, 136.4, 137.7, 161.8; IR (neat, $\mathrm{cm}^{-1}$ ) 2212, 1729, 1610, 1602; HRMS Calcd for $\mathrm{C}_{23} \mathrm{H}_{14} \mathrm{O}_{2}$ : 322.0094. Found: 322.0100. 
2-[2-(Phenylethynyl)phenyl]naphthalene (21). 2-(2-Iodophenyl)naphthalene ${ }^{3}$ and phenylacetylene were employed. Purification by flash chromatography (40:1 hexane/EtOAc) afforded $292 \mathrm{mg}(96 \%)$ of the product as a light yellow liquid: ${ }^{1} \mathrm{H}$ NMR $\left(\mathrm{CDCl}_{3}\right) \delta$ 7.24-7.58 (m, 10H), 7.70-7.72 (m, 1H), 7.86-7.97 (m, 4H), $8.17(\mathrm{~s}, 1 \mathrm{H}) ;{ }^{13} \mathrm{C} \mathrm{NMR}\left(\mathrm{CDCl}_{3}\right) \delta$ 89.7, 92.7, 122.0, 123.6, 126.2, 126.3, 127.4, 127.5, 127.9, 128.0, 128.3, 128.4, 128.5, 128.9, 130.0, 131.6, 132.9, 133.3, 133.5, 138.3, 144.0 (one carbon was missed due to overlap); IR (neat, $\mathrm{cm}^{-1}$ ) 3055, 1600, 1493; HRMS Calcd for $\mathrm{C}_{24} \mathrm{H}_{16}$ : 304.1252. Found: 304.1256.

1,4-Bis(biphen-2-ylethynyl)benzene (24). 2-Ethynylbiphenyl and 1,4-diiodobenzene were employed. Purification by flash chromatography (20:1 hexane/EtOAc) afforded $103 \mathrm{mg}$ (80\%) of the product as a white solid: $\mathrm{mp} 163-164{ }^{\circ} \mathrm{C} ;{ }^{1} \mathrm{H} \mathrm{NMR}\left(\mathrm{CDCl}_{3}\right) \delta 7.23(\mathrm{~s}, 2 \mathrm{H}), 7.34-$ $7.49(\mathrm{~m}, 6 \mathrm{H}), 7.63-7.68(\mathrm{~m}, 3 \mathrm{H}) ;{ }^{13} \mathrm{C} \mathrm{NMR}\left(\mathrm{CDCl}_{3}\right) \delta$ 91.5, 92.2, 121.6, 123.3, 127.3, 127.8, 128.1, 128.9, 129.6, 129.7, 131.4, 133.1, 140.7, 144.2; IR (neat, $\left.\mathrm{cm}^{-1}\right)$ 3062, 1512; HRMS Calcd for $\mathrm{C}_{24} \mathrm{H}_{16}$ : 304.1252. Found: 304.1256.

9-Phenyl-10-(phenylethynyl)phenanthrene (26). 9-Iodo-10-phenylphenanthrene (6) and phenylacetylene were employed. Purification by flash chromatography (40:1 hexane/EtOAc) afforded $188 \mathrm{mg}(53 \%)$ of the product as a white solid: $\mathrm{mp} 143-144{ }^{\circ} \mathrm{C} ;{ }^{1} \mathrm{H}$ NMR $\left(\mathrm{CDCl}_{3}\right) \delta$ 7.24-7.30 (m, 5H), 7.50-7.77 (m, 10H), 8.63-8.79 (m, 3H); ${ }^{13} \mathrm{C} \mathrm{NMR}\left(\mathrm{CDCl}_{3}\right) \delta$ $88.0,98.5,119.3,122.8,122.9,123.7,127.0,127.3,127.4,127.5,127.6,127.7,128.0,128.3$, 128.4, 128.5, 130.0, 130.5, 130.9, 131.0, 131.6, 131.7, 140.0, 143.2; IR (neat, $\left.\mathrm{cm}^{-1}\right) 3061,1599$; HRMS Calcd for $\mathrm{C}_{24} \mathrm{H}_{16}$ : 304.1252 . Found: 304.1256 .

4-Nitro-2'-(phenylethynyl)biphenyl (13). $\mathrm{Pd}(\mathrm{dba})_{2}(28.8 \mathrm{mg}, 5 \mathrm{~mol} \%), \mathrm{PPh}_{3}(26.0 \mathrm{mg}$, $10 \mathrm{~mol} \%), \mathrm{CsF}$ (304 mg, $2.0 \mathrm{mmol}), 2$-(phenylethynyl)phenylboronic acid (0.266 g, $1.2 \mathrm{mmol}$ ) and 1-iodo-4-nitrobenzene $(0.249 \mathrm{~g}, 1.0 \mathrm{mmol})$ in DME $(5 \mathrm{~mL})$ were heated under an $\mathrm{N}_{2}$ 
atmosphere at $80{ }^{\circ} \mathrm{C}$ for $7 \mathrm{~h}$. The mixture was allowed to cool to room temperature, diluted with diethyl ether $(50 \mathrm{~mL})$, washed with satd aq $\mathrm{NH}_{4} \mathrm{Cl}$. The organic layer was dried $\left(\mathrm{MgSO}_{4}\right)$, filtered, and the solvent was removed under reduced pressure. The residue was purified by column chromatography (7:1 hexane/EtOAc) on silica gel to afford $251 \mathrm{mg}(84 \%)$ of compound 13 as a yellow solid: mp 95-97 ${ }^{\circ} \mathrm{C} ;{ }^{1} \mathrm{H}$ NMR $\left(\mathrm{CDCl}_{3}\right) \delta 7.31(\mathrm{~s}, 5 \mathrm{H}), 7.41-7.45(\mathrm{~m}, 3 \mathrm{H})$, , 7.68$7.70(\mathrm{~m}, 1 \mathrm{H}), 7.83(\mathrm{~d}, J=6.6 \mathrm{~Hz}, 2 \mathrm{H}), 8.31(\mathrm{~d}, J=6.6 \mathrm{~Hz}, 2 \mathrm{H}) ;{ }^{13} \mathrm{C} \mathrm{NMR}\left(\mathrm{CDCl}_{3}\right) \delta 88.3,93.2$, $121.7,122.9,123.3,128.5,128.6,128.8,129.4,130.3,131.4,133.3,141.3,147.3$ (missing two $\mathrm{sp}^{2}$ carbons due to overlap); IR (neat, $\mathrm{cm}^{-1}$ ) 3062, 1599, 1516; HRMS Calcd for $\mathrm{C}_{20} \mathrm{H}_{13} \mathrm{NO}_{2}$ : 299.0946. Found: 299.0950 .

\section{General procedure for the electrophilic cyclization of 2-(arylethynyl)biphenyls by}

ICl. To a solution of 2-(arylethynyl)biphenyl $(0.30 \mathrm{mmol})$ in $\mathrm{CH}_{2} \mathrm{Cl}_{2}(3 \mathrm{~mL})$ under $\mathrm{N}_{2}$ was added $\mathrm{ICl}$ (1.2 equiv) in $\mathrm{CH}_{2} \mathrm{Cl}_{2}(0.5 \mathrm{~mL})$ at $-78{ }^{\circ} \mathrm{C}$. The reaction mixture was stirred at $-78{ }^{\circ} \mathrm{C}$ for $1 \mathrm{~h}$ unless otherwise indicated. The reaction mixture was then diluted with diethyl ether (50 $\mathrm{mL})$, washed with $25 \mathrm{~mL}$ of satd aq $\mathrm{Na}_{2} \mathrm{~S}_{2} \mathrm{O}_{3}$, dried $\left(\mathrm{MgSO}_{4}\right)$, and filtered. The solvent was evaporated under reduced pressure and the product was purified by chromatography on a silica gel column.

9-Iodo-10-(4-methoxyphenyl)phenanthrene (2). Purification by flash chromatography (30:1 hexane/EtOAc) afforded $122 \mathrm{mg}(99 \%)$ of the product as a white solid: $\mathrm{mp} 170-171^{\circ} \mathrm{C}$; ${ }^{1} \mathrm{H} \mathrm{NMR}\left(\mathrm{CDCl}_{3}\right) \delta 3.94(\mathrm{~s}, 3 \mathrm{H}), 7.09(\mathrm{dd}, J=2.1,6.6 \mathrm{~Hz}, 2 \mathrm{H}), 7.21(\mathrm{dd}, J=2.1,6.6 \mathrm{~Hz}, 2 \mathrm{H})$, 7.40-7.49 (m, 2H), 7.64-7.72 (m, 3H), 8.45-8.49 (m, 1H), 8.67-8.78 (m, 2H); ${ }^{13} \mathrm{C} \mathrm{NMR}\left(\mathrm{CDCl}_{3}\right)$ $\delta 55.6,107.7,114.0,122.8,122.9,127.2,127.3,127.7,128.3,129.0,130.5,130.8,131.3,132.7$, 132.9, 135.0, 138.2, 145.3, 159.4; IR (neat, $\mathrm{cm}^{-1}$ ) 3066, 3024, 2834, 1610; HRMS Calcd for $\mathrm{C}_{21} \mathrm{H}_{15} \mathrm{IO}:$ 410.0168. Found: 410.0172. 
9-Iodo-10-(4-methylphenyl)phenanthrene (4). Purification by flash chromatography (30:1 hexane/EtOAc) afforded $116 \mathrm{mg}(98 \%)$ of the product as a yellow solid: $\mathrm{mp} 179-181^{\circ} \mathrm{C}$; ${ }^{1} \mathrm{H} \mathrm{NMR}\left(\mathrm{CDCl}_{3}\right) \delta 2.52(\mathrm{~s}, 3 \mathrm{H}), 7.18(\mathrm{dd}, J=1.5,6.3 \mathrm{~Hz}, 2 \mathrm{H}), 7.36-7.44(\mathrm{~m}, 4 \mathrm{H}), 7.64-7.76(\mathrm{~m}$, $3 \mathrm{H}), 8.46-8.49(\mathrm{~m}, 1 \mathrm{H}), 8.66-8.78(\mathrm{~m}, 2 \mathrm{H}) ;{ }^{13} \mathrm{C} \mathrm{NMR}\left(\mathrm{CDCl}_{3}\right) \delta$ 21.7, 107.0, 122.8, 122.9, 127.2, 127.3, 127.7, 128.3, 129.0, 129.4, 130.0, 130.5, 130.8, 132.8, 134.9, 137.8, 142.7, 145.6 (missing one $\mathrm{sp}^{2}$ carbon due to overlap); IR (neat, $\mathrm{cm}^{-1}$ ) 3067, 3020, 2917; HRMS Calcd for $\mathrm{C}_{21} \mathrm{H}_{15} \mathrm{I}$ : 394.0219. Found: 394.0226.

9-Iodo-10-phenylphenanthrene (6). Purification by flash chromatography (40:1 hexane/EtOAc) afforded $112 \mathrm{mg}(99 \%)$ of the product as a white solid with a melting point and spectral properties identical to those previously reported. ${ }^{2}$

Ethyl 4-(10-iodo-9-phenanthryl)benzoate (8). The reaction mixture was stirred at room temperature for $1 \mathrm{~h}$. Purification by flash chromatography (15:1 hexane/EtOAc) afforded $136 \mathrm{mg}(100 \%)$ of the product as a white solid: $\mathrm{mp} 152-153{ }^{\circ} \mathrm{C} ;{ }^{1} \mathrm{H} \mathrm{NMR}\left(\mathrm{CDCl}_{3}\right) \delta 1.46(\mathrm{t}, J=$ $7.2 \mathrm{~Hz}, 3 \mathrm{H}), 4.47(\mathrm{q}, J=7.2 \mathrm{~Hz}, 2 \mathrm{H}), 7.30-7.45(\mathrm{~m}, 4 \mathrm{H}), 7.66-7.75(\mathrm{~m}, 3 \mathrm{H}), 8.26(\mathrm{dd}, J=1.8$, 6.6 Hz, 2H), 8.45-8.49 (m, 1H), 8.68-8.78 (m, 2H); ${ }^{13} \mathrm{C} \mathrm{NMR}\left(\mathrm{CDCl}_{3}\right) \delta$ 14.6, 61.4, 106.0, 122.9, 123.0, 127.4, 127.5, 128.0, 128.4, 128.5, 130.1, 130.3, 130.4, 130.5, 130.8, 132.1, 132.5, 134.9, 144.6, 150.0, 166.7; IR (neat, $\mathrm{cm}^{-1}$ ) 3069, 2979, 1714; HRMS Calcd for $\mathrm{C}_{23} \mathrm{H}_{17} \mathrm{IO}_{2}: 452.0273$. Found: 452.0278.

9-Iodo-10-(4-nitrophenyl)phenanthrene (10). Purification by flash chromatography (7:1 hexane/EtOAc) afforded an inseparable mixture of the desired compound $10(57 \%)$ and ICl alkyne adducts (42\%) (yields were calculated by ${ }^{1} \mathrm{H}$ NMR spectroscopic analysis). Recrystallization from hexanes/ethyl acetate afforded $31 \mathrm{mg}(30 \%)$ of the desired product $\mathbf{1 0}$ as a yellow solid: mp $205-206{ }^{\circ} \mathrm{C} ;{ }^{1} \mathrm{H}$ NMR $\left(\mathrm{CDCl}_{3}\right) \delta$ 7.23-7.26 (m, $\left.1 \mathrm{H}\right), 7.43-7.51$ (m, 3H), 7.68- 
$7.77(\mathrm{~m}, 3 \mathrm{H}), 8.43-8.46(\mathrm{~m}, 3 \mathrm{H}), 8.70-8.79(\mathrm{~m}, 2 \mathrm{H}) ;{ }^{13} \mathrm{C} \mathrm{NMR}\left(\mathrm{CDCl}_{3}\right)$ \& 105.9, 123.0, 123.2, 124.2, 127.6, 127.8, 128.0, 128.4, 128.6, 130.6, 130.9, 131.5, 131.7, 132.2, 134.9, 143.2, 147.8, 152.1; IR (neat, $\mathrm{cm}^{-1}$ ) 3070, 1599, 1516; HRMS Calcd for $\mathrm{C}_{20} \mathrm{H}_{12} \mathrm{INO}_{2}$ : 424.9913. Found: 424.9919.

9-Iodo-10-phenylphenanthrene-2-carbaldehyde (12). Purification by flash chromatography (5:1 hexane/EtOAc) afforded an inseparable mixture of the desired compound $12(71 \%)$ and $\mathrm{ICl}$ alkyne adducts (17\%) (yields were calculated by ${ }^{1} \mathrm{H}$ NMR spectroscopic analysis). Recrystallization from hexanes/ethyl acetate afforded $49 \mathrm{mg}(40 \%)$ of the desired product 12 as a white solid: $\mathrm{mp} 121-123{ }^{\circ} \mathrm{C} ;{ }^{1} \mathrm{H} \mathrm{NMR}\left(\mathrm{CDCl}_{3}\right) \delta$ 7.30-7.33 (m, 2H), 7.58-7.61 (m, 3H), 7.76-7.79 (m, 2H), $7.88(\mathrm{~s}, 1 \mathrm{H}), 8.16(\mathrm{dd}, J=1.5,8.4 \mathrm{~Hz}, 1 \mathrm{H}), 8.50-8.53(\mathrm{~m}, 1 \mathrm{H}), 8.71-$ $8.74(\mathrm{~m}, 1 \mathrm{H}), 8.84(\mathrm{~d}, J=8.7 \mathrm{~Hz}, 1 \mathrm{H}), 9.96(\mathrm{~s}, 1 \mathrm{H}) ;{ }^{13} \mathrm{C} \mathrm{NMR}\left(\mathrm{CDCl}_{3}\right) \delta$ 108.3, 123.8, 124.0, $124.9,128.3,128.6,129.0,129.8,130.0,130.2,132.2,133.8,133.9,134.5,134.8,135.2,144.7$, 145.7, 192.2; IR (neat, $\mathrm{cm}^{-1}$ ) 3059, 3024, 1694, 1606; HRMS Calcd for $\mathrm{C}_{21} \mathrm{H}_{13} \mathrm{IO}: 408.0011$. Found: 408.0018.

9-Iodo-2-nitro-10-phenylphenanthrene (14). Purification by flash chromatography (7:1 hexane/EtOAc) afforded $70 \mathrm{mg}(55 \%)$ of the product as a yellow solid: $\mathrm{mp} 193-194{ }^{\circ} \mathrm{C} ;{ }^{1} \mathrm{H}$ NMR $\left(\mathrm{CDCl}_{3}\right) \delta$ 7.23-7.30 (m, 2H), 7.59-7.62 (m, 3H), 7.79-7.82 (m, 2H), $8.30(\mathrm{~s}, 1 \mathrm{H}), 8.40-$ $8.44(\mathrm{~m}, 1 \mathrm{H}), 8.52-8.55(\mathrm{~m}, 1 \mathrm{H}), 8.66-8.74(\mathrm{~m}, 1 \mathrm{H}), 8.81-8.89(\mathrm{~m}, 1 \mathrm{H}) ;{ }^{13} \mathrm{C} \mathrm{NMR}\left(\mathrm{CDCl}_{3}\right) \delta$ 109.4, 120.9, 123.9, 124.4, 124.6, 128.7, 128.9, 129.1, 129.4, 130.0, 130.3, 132.0, 134.0, 134.4, 135.4, 144.1, 145.6, 146.4; IR (neat, $\mathrm{cm}^{-1}$ ) 3083, 1536, 1513; HRMS Calcd for $\mathrm{C}_{20} \mathrm{H}_{12} \mathrm{INO}_{2}$ : 424.9913. Found: 424.9919. Anal. Calcd for $\mathrm{C}_{20} \mathrm{H}_{12} \mathrm{INO}_{2}$ : C, 56.48; H, 2.84; N, 3.29. Found: C, 56.14; H, 2.56; N, 3.17. 
5-Iodo-6-phenylbenzo $[b]$ naphtho[1,2- $d]$ furan (16). The reaction mixture was stirred at $-78{ }^{\circ} \mathrm{C}$ for 5 h. Purification by flash chromatography (50:1 hexane/EtOAc) afforded $113 \mathrm{mg}$ $(91 \%)$ of the product as a light yellow liquid: ${ }^{1} \mathrm{H} \mathrm{NMR}\left(\mathrm{CDCl}_{3}\right) \delta 7.45-7.69(\mathrm{~m}, 9 \mathrm{H}), 7.77(\mathrm{t}, J=$ $7.8 \mathrm{~Hz}, 1 \mathrm{H}), 8.41-8.44(\mathrm{~m}, 1 \mathrm{H}), 8.54(\mathrm{~d}, J=8.7 \mathrm{~Hz}, 1 \mathrm{H}), 8.64(\mathrm{~d}, J=8.1 \mathrm{~Hz}, 1 \mathrm{H}) ;{ }^{13} \mathrm{C} \mathrm{NMR}$ $\left(\mathrm{CDCl}_{3}\right) \delta 105.0,112.6,118.2,122.4,123.7,124.0,124.7,126.6,126.8,128.0,128.6,128.7$, 128.9, 130.5, 131.9, 134.3, 135.1, 140.6, 152.2, 156.1; IR (neat, $\mathrm{cm}^{-1}$ ) 3058, 2961; HRMS Calcd for $\mathrm{C}_{22} \mathrm{H}_{13} \mathrm{IO}:$ 420.0011. Found: 420.0021 .

9-Iodo-10-(2-thiophenyl)phenanthrene (18). Purification by flash chromatography (30:1 hexane/EtOAc) afforded $111 \mathrm{mg}(96 \%)$ of the product as a white solid: $\mathrm{mp} 140-142{ }^{\circ} \mathrm{C}$; ${ }^{1} \mathrm{H}$ NMR $\left(\mathrm{CDCl}_{3}\right) \delta$ 7.06-7.08 (m, 1H), 7.23-7.26 (m, 1H), 7.45-7.76 (m, 6H), 8.44-8.49 (m, 1H), 8.66-8.75 (m, 2H); ${ }^{13} \mathrm{C}$ NMR $\left(\mathrm{CDCl}_{3}\right) \delta 110.5,122.7,122.9,126.5,127.2,127.5,128.2,128.4$, $128.7,128.8,130.3,131.1,132.6,133.2,135.3,138.4,146.5 ;$ IR (neat, $\mathrm{cm}^{-1}$ ) 2925, 1464, 1216; HRMS Calcd for $\mathrm{C}_{18} \mathrm{H}_{11}$ IS: 385.9626 . Found: 385.9631.

7-Iodo-8-phenyl-5H-dibenzo[c, $f]$ chromen-5-one (20). Purification by flash chromatography (3:1 hexane/EtOAc) afforded $87 \mathrm{mg}(65 \%)$ of the product as a white solid: $\mathrm{mp}$ 205-207 ${ }^{\circ} \mathrm{C} ;{ }^{1} \mathrm{H}$ NMR $\left(\mathrm{CDCl}_{3}\right)$ d 7.25-7.29 (m, 2H), 7.36-7.42 (m, 1H), 7.51-7.70 (m, 6H), 8.92 $(\mathrm{t}, J=7.8 \mathrm{~Hz}, 1 \mathrm{H}), 8.53(\mathrm{~d}, J=7.2 \mathrm{~Hz}, 1 \mathrm{H}), 8.62(\mathrm{~d}, J=7.6 \mathrm{~Hz}, 1 \mathrm{H}), 8.75(\mathrm{~d}, J=7.0 \mathrm{~Hz}, 1 \mathrm{H})$; ${ }^{13} \mathrm{C} \mathrm{NMR}\left(\mathrm{CDCl}_{3}\right) \delta 92.8,113.3,122.9,125.4,126.1,127.1,127.9,128.5,128.8,128.91,128.93$, 129.5, 129.9, 130.9, 131.7, 134.6, 135.0, 143.2, 148.0, 149.0, 160.9; IR (neat, $\mathrm{cm}^{-1}$ ) 3431, 1744; HRMS Calcd for $\mathrm{C}_{23} \mathrm{H}_{13} \mathrm{IO}_{3}$ : 447.9960. Found: 447.9967.

6-Iodo-5-phenylchrysene (22). Purification by flash chromatography (40:1 hexane/EtOAc) afforded $98 \mathrm{mg}(76 \%)$ of the product as a light yellow solid: $\mathrm{mp} 168-169{ }^{\circ} \mathrm{C} ;{ }^{1} \mathrm{H}$ $\operatorname{NMR}\left(\mathrm{CDCl}_{3}\right) \delta 7.07(\mathrm{t}, J=6.9 \mathrm{~Hz}, 1 \mathrm{H}), 7.33-7.57(\mathrm{~m}, 7 \mathrm{H}), 7.71-7.76(\mathrm{~m}, 2 \mathrm{H}), 8.89(\mathrm{~d}, J=6.5$ 
$\mathrm{Hz}, 1 \mathrm{H}), 8.04(\mathrm{~d}, J=7.6 \mathrm{~Hz}, 1 \mathrm{H}), 8.56-8.60(\mathrm{~m}, 1 \mathrm{H}), 8.78(\mathrm{~d}, J=7.0 \mathrm{~Hz}, 2 \mathrm{H}) ;{ }^{13} \mathrm{C} \mathrm{NMR}\left(\mathrm{CDCl}_{3}\right)$

ठ $111.5,121.3,123.7,125.4,126.1,127.7,128.2,128.4,128.6,128.9,129.0,129.2,129.3,130.4$, 130.7, 130.8, 131.1, 133.5, 133.8, 135.3, 144.3, 150.0; IR (neat, $\mathrm{cm}^{-1}$ ) 2922; HRMS Calcd for $\mathrm{C}_{24} \mathrm{H}_{15} \mathrm{I}$ : 430.0219. Found: 430.0025 .

5-Iodo-6-phenylchrysene (23). Purification by flash chromatography (40:1

hexane/EtOAc) afforded $18 \mathrm{mg}(14 \%)$ of the product as a white solid: $\mathrm{mp} 174-176{ }^{\circ} \mathrm{C} ;{ }^{1} \mathrm{H}$ NMR $\left(\mathrm{CDCl}_{3}\right) \delta 7.12(\mathrm{t}, J=7.2 \mathrm{~Hz}, 1 \mathrm{H}), 7.30-7.33(\mathrm{~m}, 3 \mathrm{H}), 7.49-7.61(\mathrm{~m}, 5 \mathrm{H}), 7.70-7.78(\mathrm{~m}, 2 \mathrm{H})$, $8.39(\mathrm{~d}, J=7.2 \mathrm{~Hz}, 1 \mathrm{H}), 8.55-8.68(\mathrm{~m}, 2 \mathrm{H}), 8.87(\mathrm{~s}, 1 \mathrm{H}) ;{ }^{13} \mathrm{C} \mathrm{NMR}\left(\mathrm{CDCl}_{3}\right) \delta$ 111.7, 121.8, 123.5, 124.9, 126.0, 127.0, 128.0, 128.1, 128.3, 128.8, 129.1, 129.2, 129.8, 130.2, 130.8, 131.0, 131.8, 132.9, 133.7, 135.4, 143.8, 149.6; IR (neat, $\mathrm{cm}^{-1}$ ) 3057, 2920; HRMS Calcd for $\mathrm{C}_{24} \mathrm{H}_{15} \mathrm{I}$ : 430.0219. Found: 430.0025 .

1,4-Bis(10-iodophenanthr-9-yl)benzene (25). Filtration afforded $184 \mathrm{mg}(90 \%)$ of the product as a white solid: $\mathrm{mp} 328-331{ }^{\circ} \mathrm{C} ;{ }^{1} \mathrm{H} \mathrm{NMR}\left(\mathrm{CDCl}_{3}\right)$ 7.50-7.79 (m, 14H), 8.54-8.57 (m, $2 \mathrm{H}), 8.74-8.83(\mathrm{~m}, 4 \mathrm{H})$; the ${ }^{13} \mathrm{C}$ NMR and IR spectra could not be obtained due to the poor solubility of this compound in common organic solvents. HRMS Calcd for $\mathrm{C}_{34} \mathrm{H}_{20} \mathrm{I}_{2}: 681.9655$. Found: 681.9667. Anal. Calcd for $\mathrm{C}_{34} \mathrm{H}_{20} \mathrm{I}_{2}$ : C, 59.85; H, 2.95. Found: C, 59.43; H, 2.52.

9,10-Diphenylbenzo[g]chrysene (27). $\mathrm{Pd}(\mathrm{OAc})_{2}(2.8 \mathrm{mg}, 5 \mathrm{~mol} \%)$, NaOAc (41 mg, 0.5 mmol), LiCl (31.5 mg, $0.75 \mathrm{mmol}$ ), DMF (5 mL), 9-iodo-10-phenylphenanthrene (95 mg, 0.25 $\mathrm{mmol})$ and diphenylacetylene $(44.5 \mathrm{mg}, 0.25 \mathrm{mmol})$ were placed in a vial. The resulting mixture was heated under an $\mathrm{N}_{2}$ atmosphere at $100{ }^{\circ} \mathrm{C}$ for $5 \mathrm{~d}$. The mixture was allowed to cool to room temperature, diluted with diethyl ether $(50 \mathrm{~mL})$, washed with satd aq $\mathrm{NH}_{4} \mathrm{Cl}(25 \mathrm{~mL})$, dried $\left(\mathrm{MgSO}_{4}\right)$, and filtered. The solvent was removed under reduced pressure and the residue was purified by column chromatography (100:1 hexane/EtOAc) on silica gel to afford $89 \mathrm{mg}(83 \%)$ 
of the product as a white solid: $\mathrm{mp} 228-230{ }^{\circ} \mathrm{C} ;{ }^{1} \mathrm{H}$ NMR $\left(\mathrm{CDCl}_{3}\right) \delta$ 7.04-7.10 (m, 6H), 7.17$7.30(\mathrm{~m}, 5 \mathrm{H}), 7.40-7.51(\mathrm{~m}, 2 \mathrm{H}), 7.57-7.80(\mathrm{~m}, 5 \mathrm{H}), 8.58(\mathrm{~d}, J=7.6 \mathrm{~Hz}, 1 \mathrm{H}), 8.69(\mathrm{~d}, J=7.0 \mathrm{~Hz}$ $1 \mathrm{H}), 8.83(\mathrm{t}, J=6.8 \mathrm{~Hz}, 2 \mathrm{H}) ;{ }^{13} \mathrm{C} \mathrm{NMR}\left(\mathrm{CDCl}_{3}\right) \delta$ 123.2, 123.8, 125.7, 125.8, 126.27, 126.29, $126.41,126.66,126.69,127.0,127.1,127.8,128.03,128.06,128.07,129.0,129.5,129.7,130.05$, 130.08, 130.87, 130.98, 131.4, 131.7, 132.1, 132.5, 136.1, 138.5, 139.7, 143.0; IR (neat, $\mathrm{cm}^{-1}$ )

3062, 2925; HRMS Calcd for $\mathrm{C}_{34} \mathrm{H}_{22}$ : 430.1722. Found: 430.1729 .

\section{References}

1. $\quad$ Campo, M. A.; Larock, R. C. J. Org. Chem. 2002, 67, 5616.

2. Campo, M. A.; Larock, R. C. Org. Lett. 2000, 2, 3675.

3. Hart, H.; Harada, K.; Du, C. J. F. J. Org. Chem. 1985, 50, 3104.

4. John, J. A.; Tour, J. M. Tetrahedron 1997, 53, 15515.

5. $\quad$ Letsinger, R. L.; Feare, T. E.; Savereide, T. J.; Nazy, J. R. J. Org. Chem. 1961, $26,1271$.

6. Larock, R. C.; Doty, M. J.; Han, X. J. Org. Chem. 1999, 64, 8770.

7. Kamikawa, T.; Hayashi, T. J. Org. Chem. 1998, 63, 8922. 


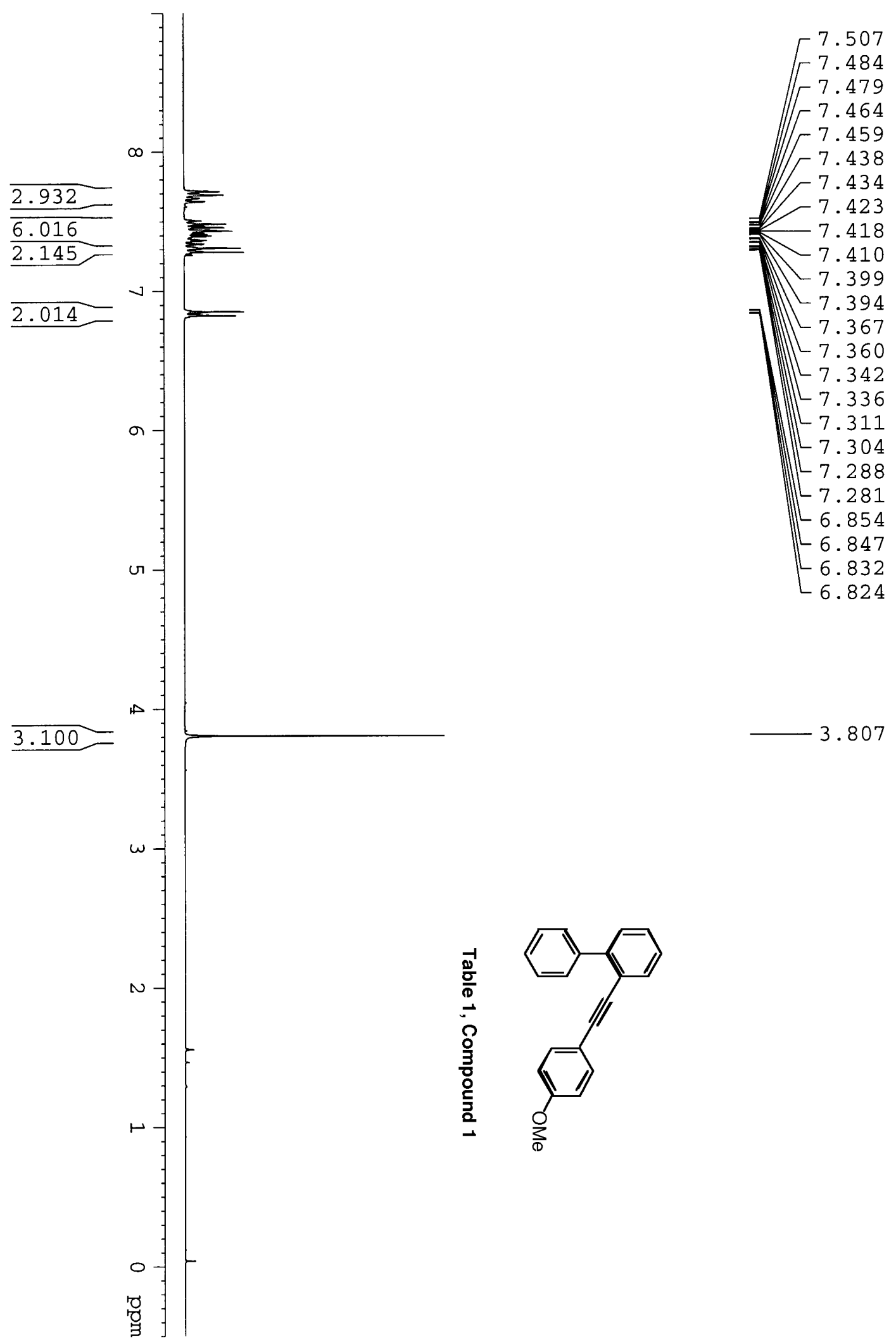




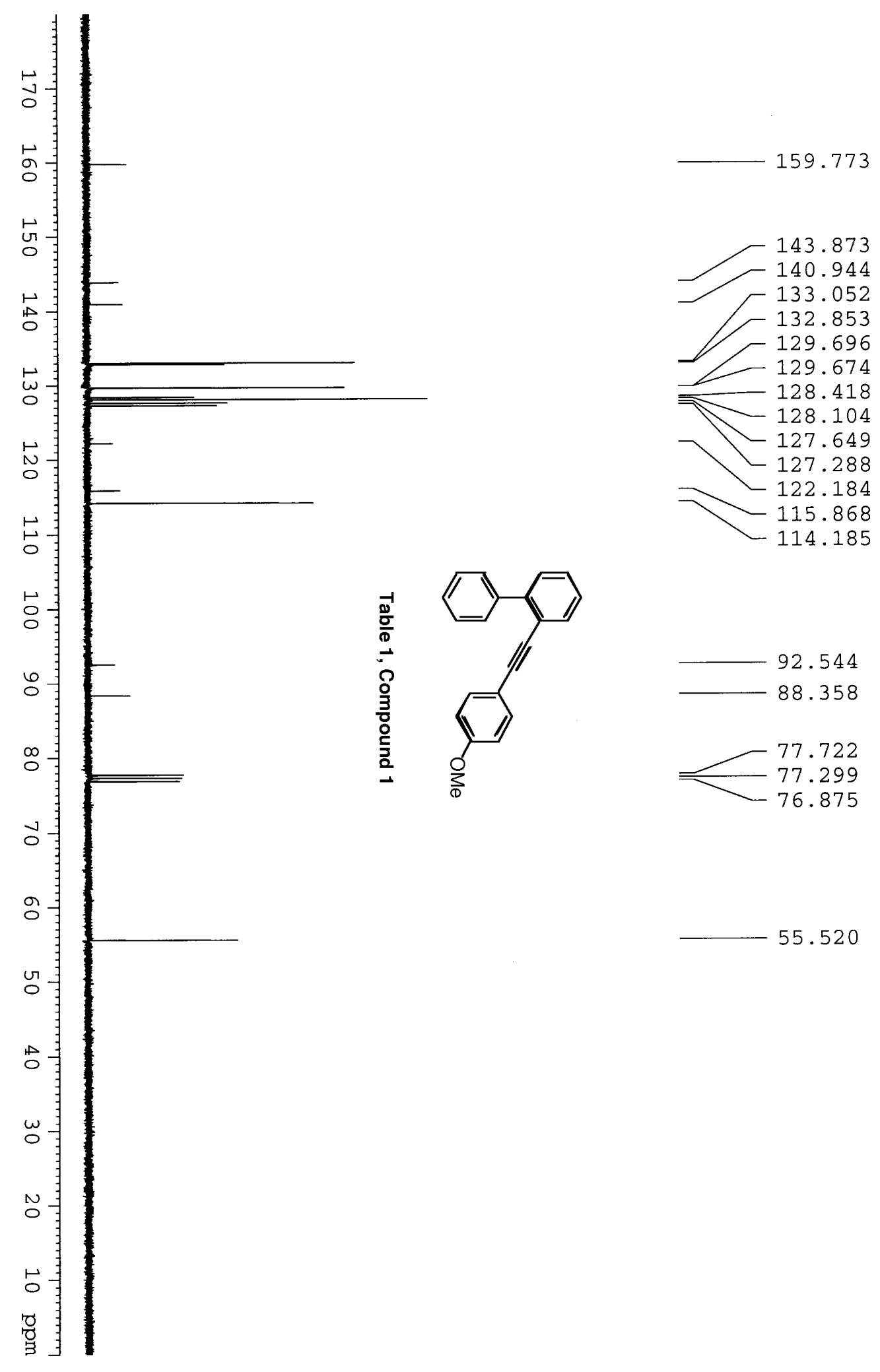



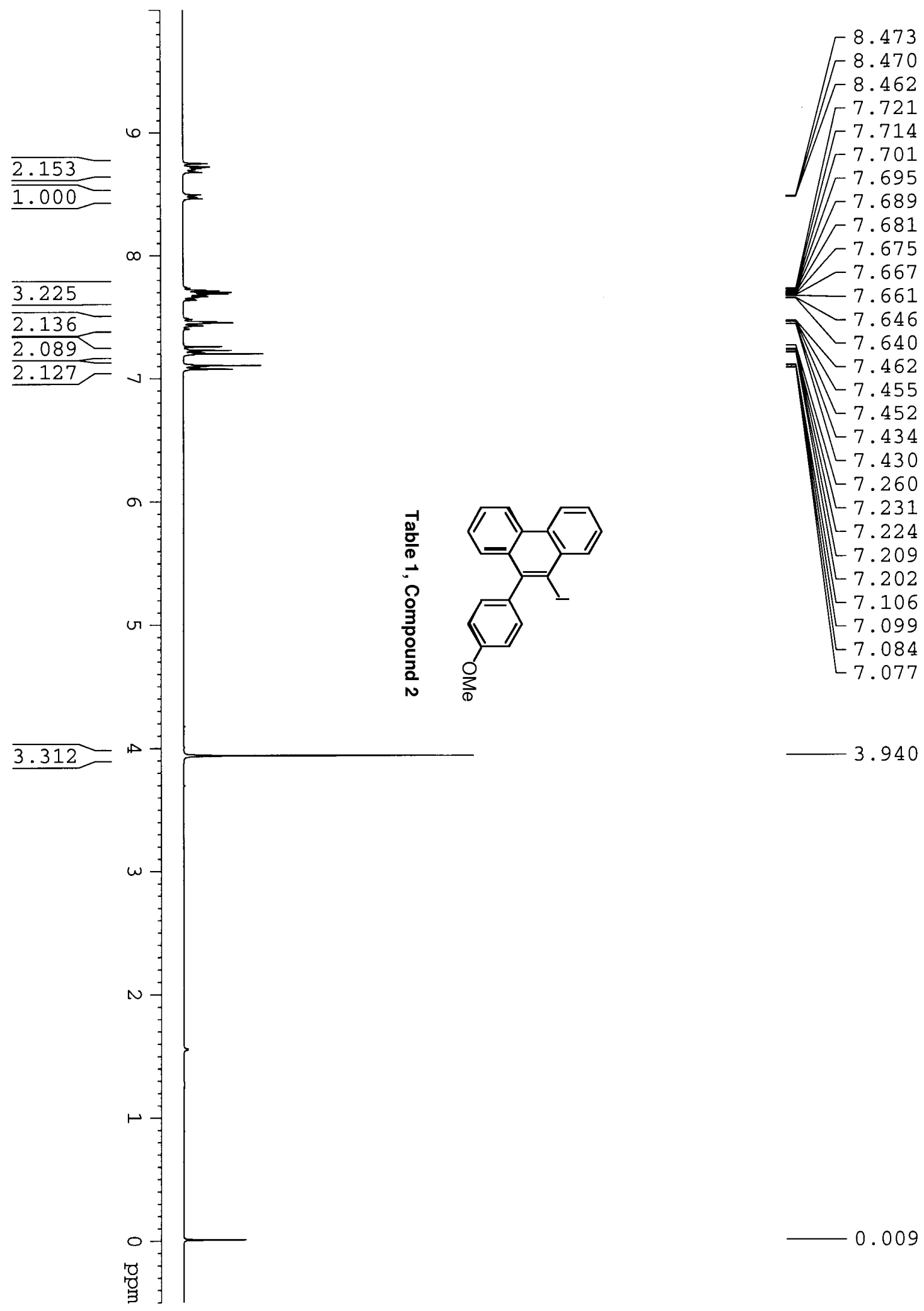


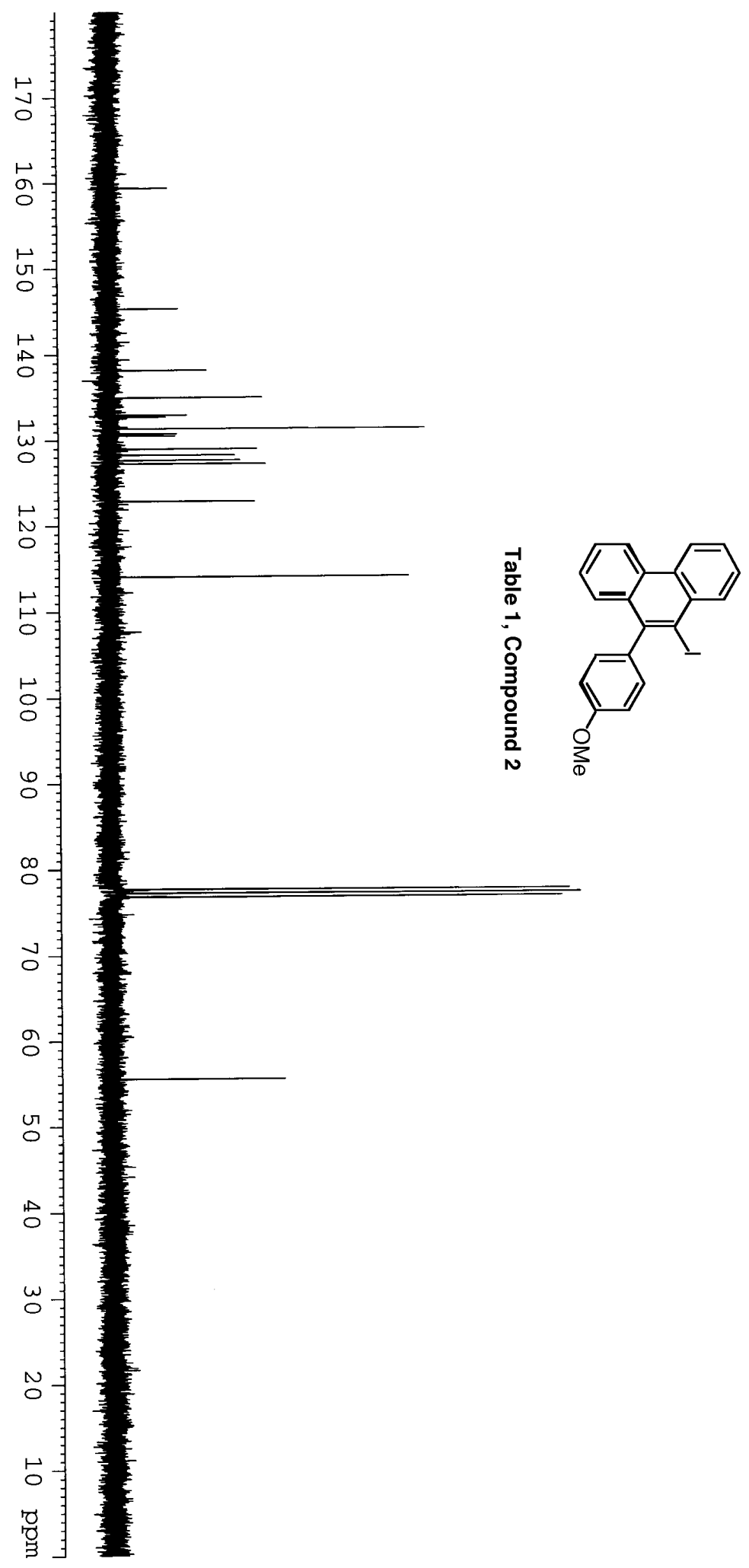

159.382

145.316

138.163

134.994

$-132.731$

131.331

$-130.785$

130.505

$-128.998$

$-128.270$

$-127.685$

$-127.270$

$-127.253$

$-122.855$

$-122.822$

114.047

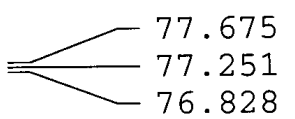

55.554 

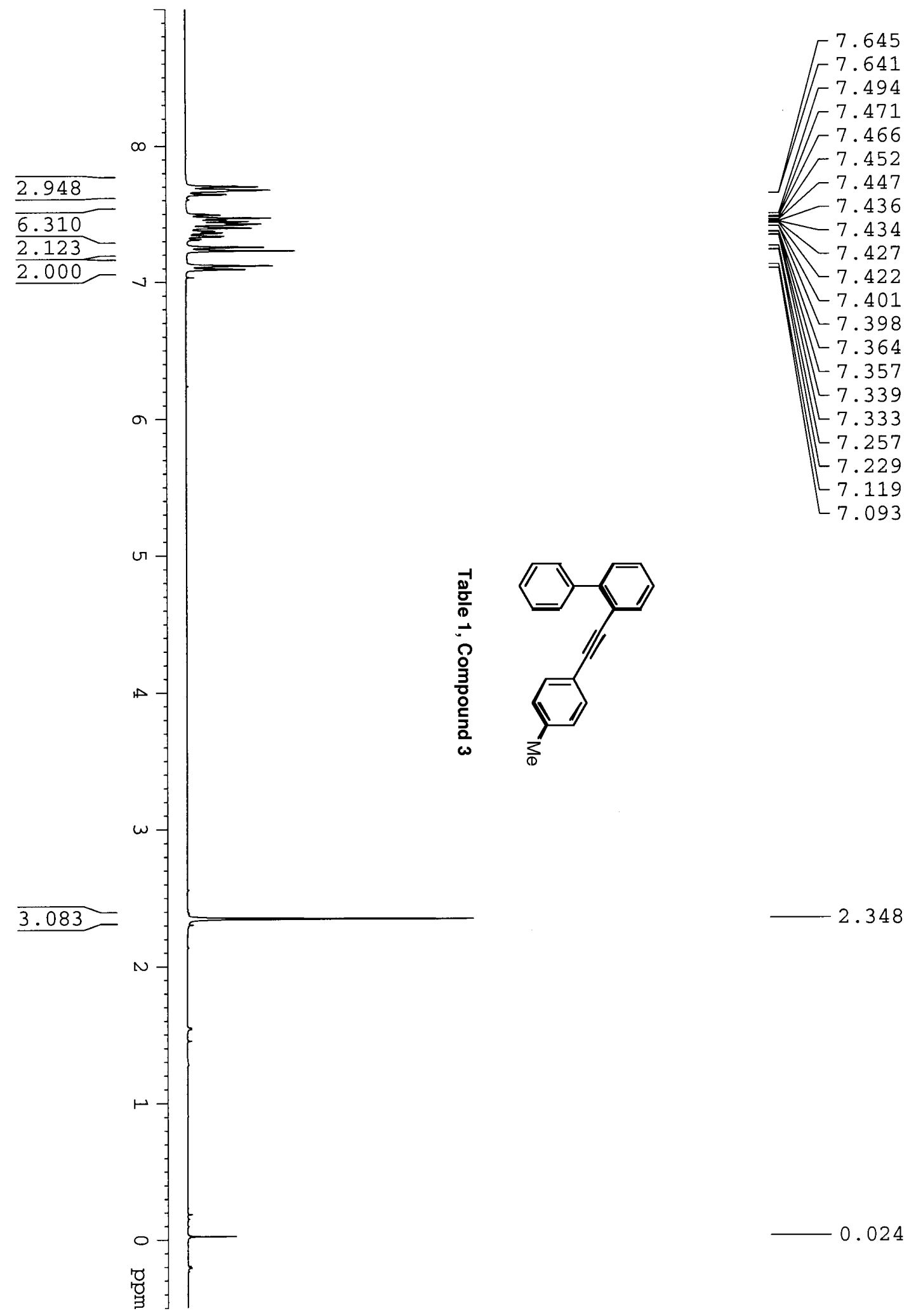

7.641

7.494

7.466

7.452

7.447

7.434

7.401

$-7.364$

7.333

7.229

7.093

2.348

0.024 

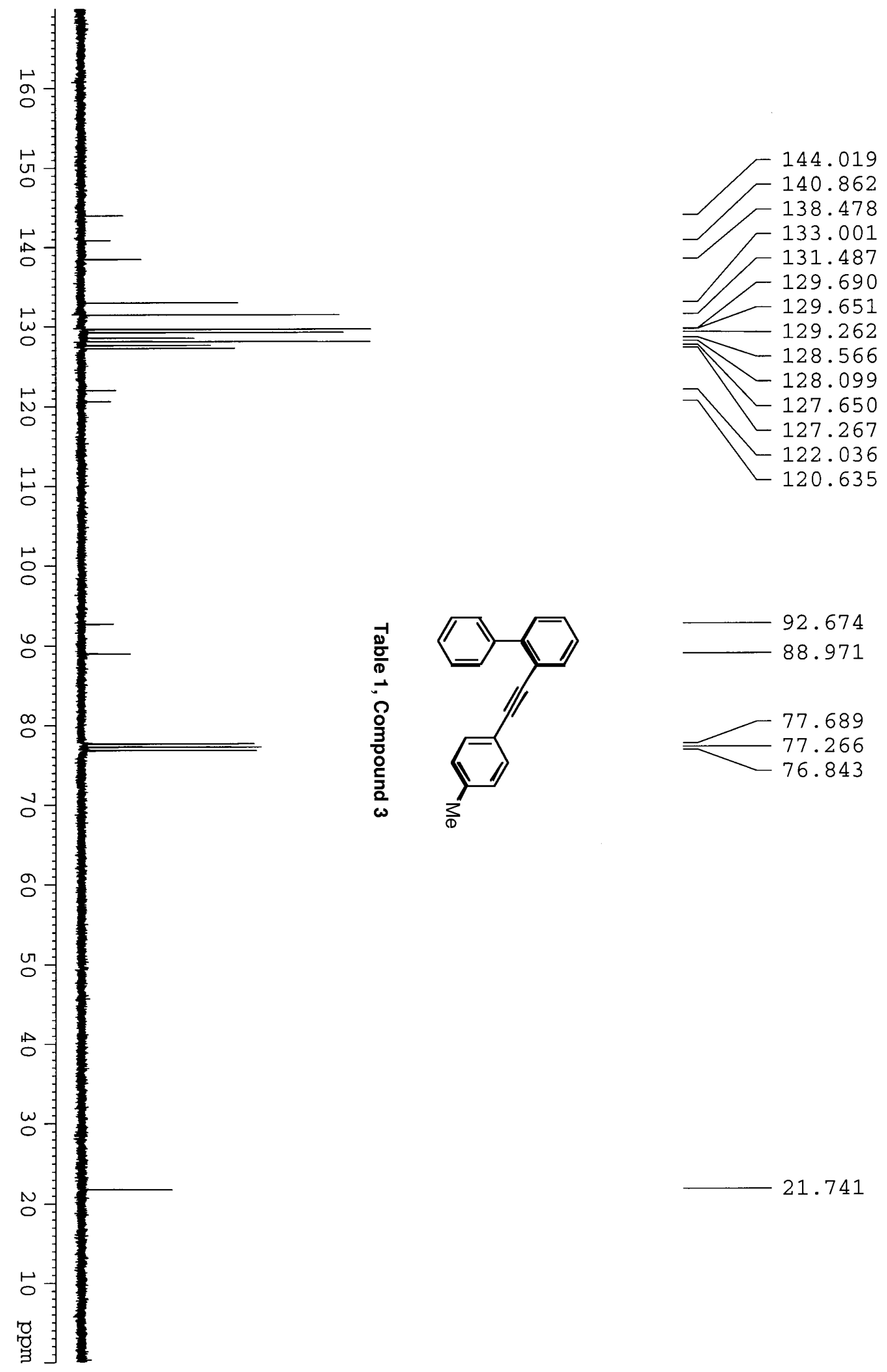

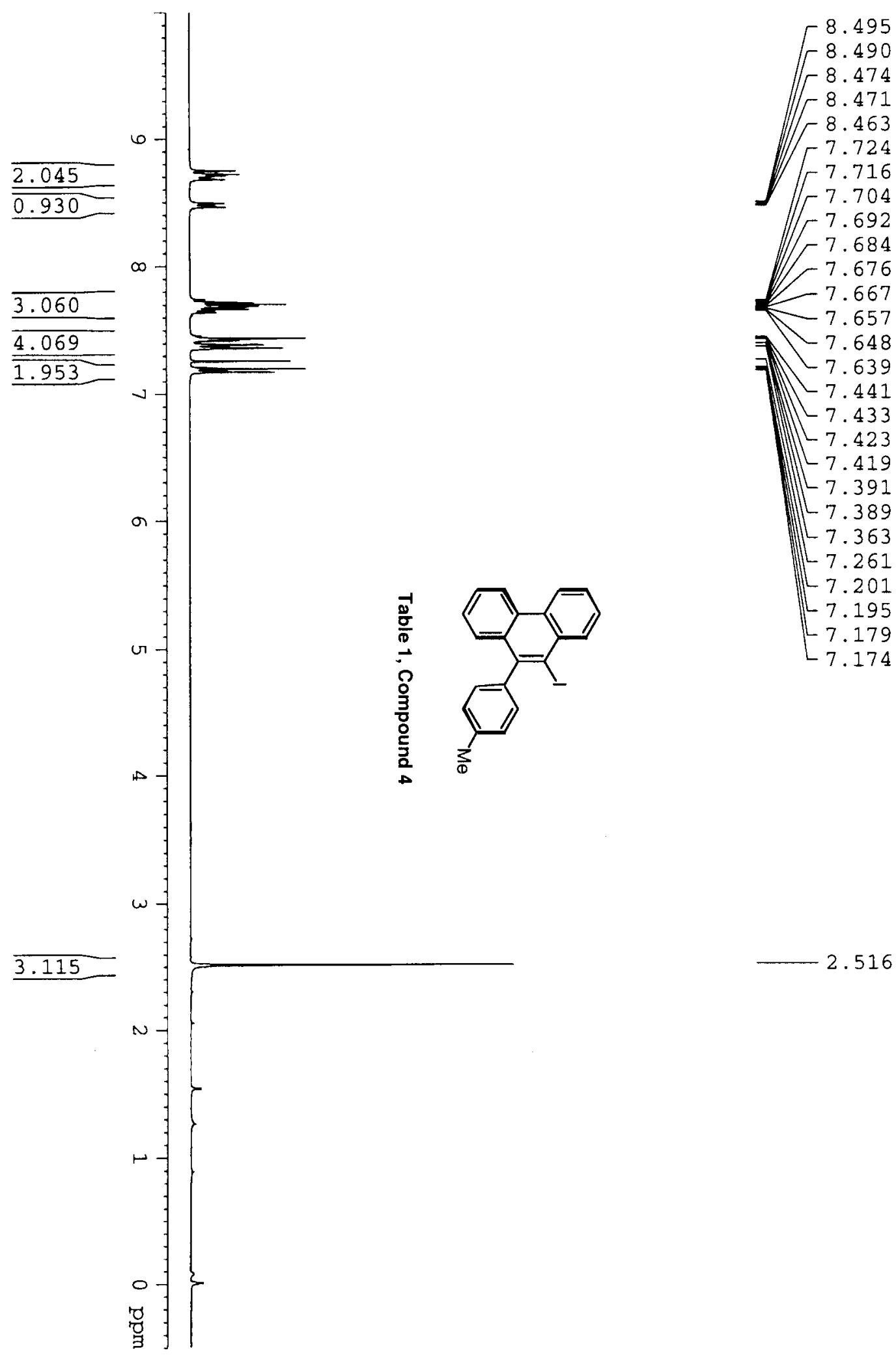

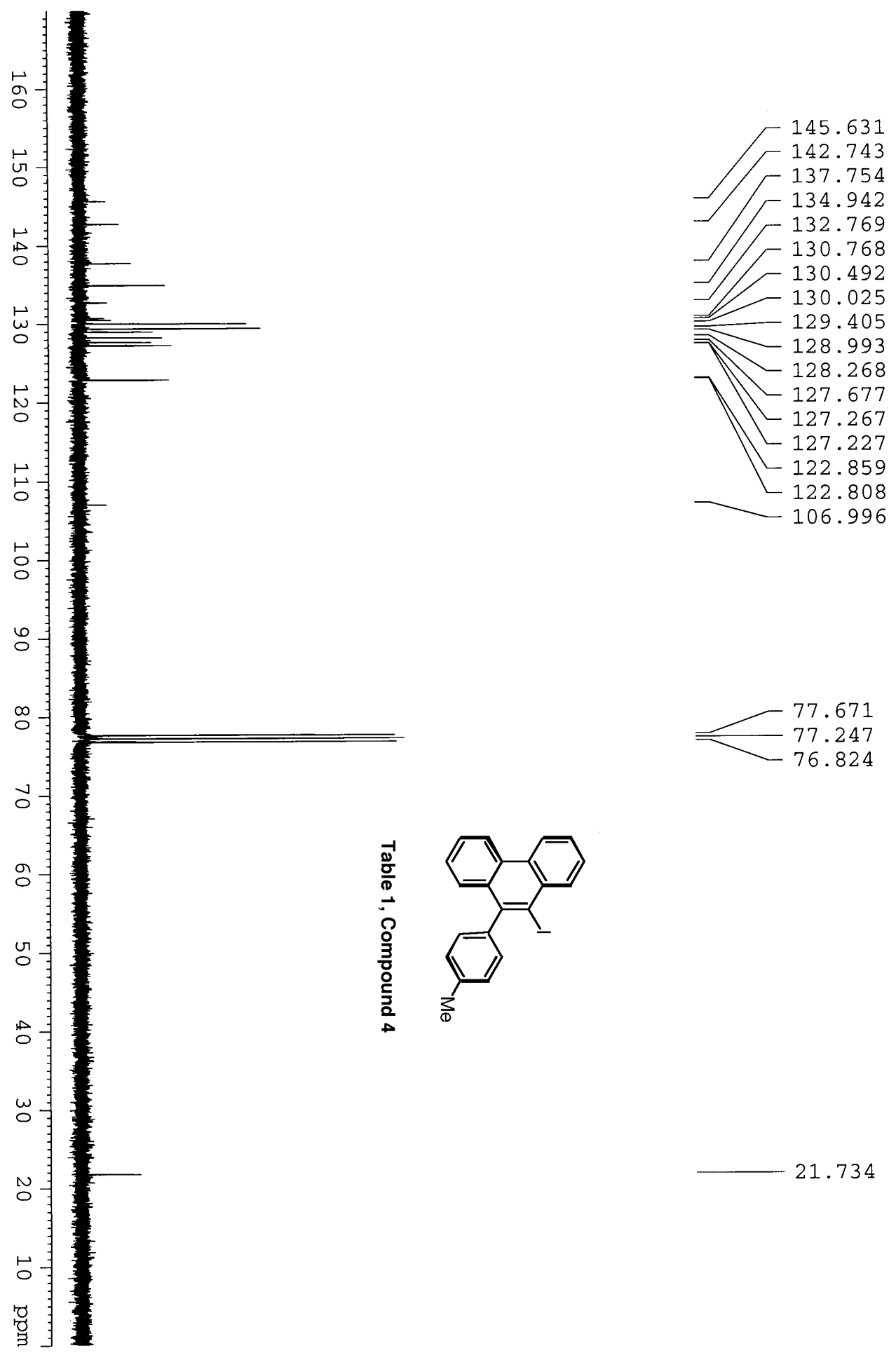

77.247 

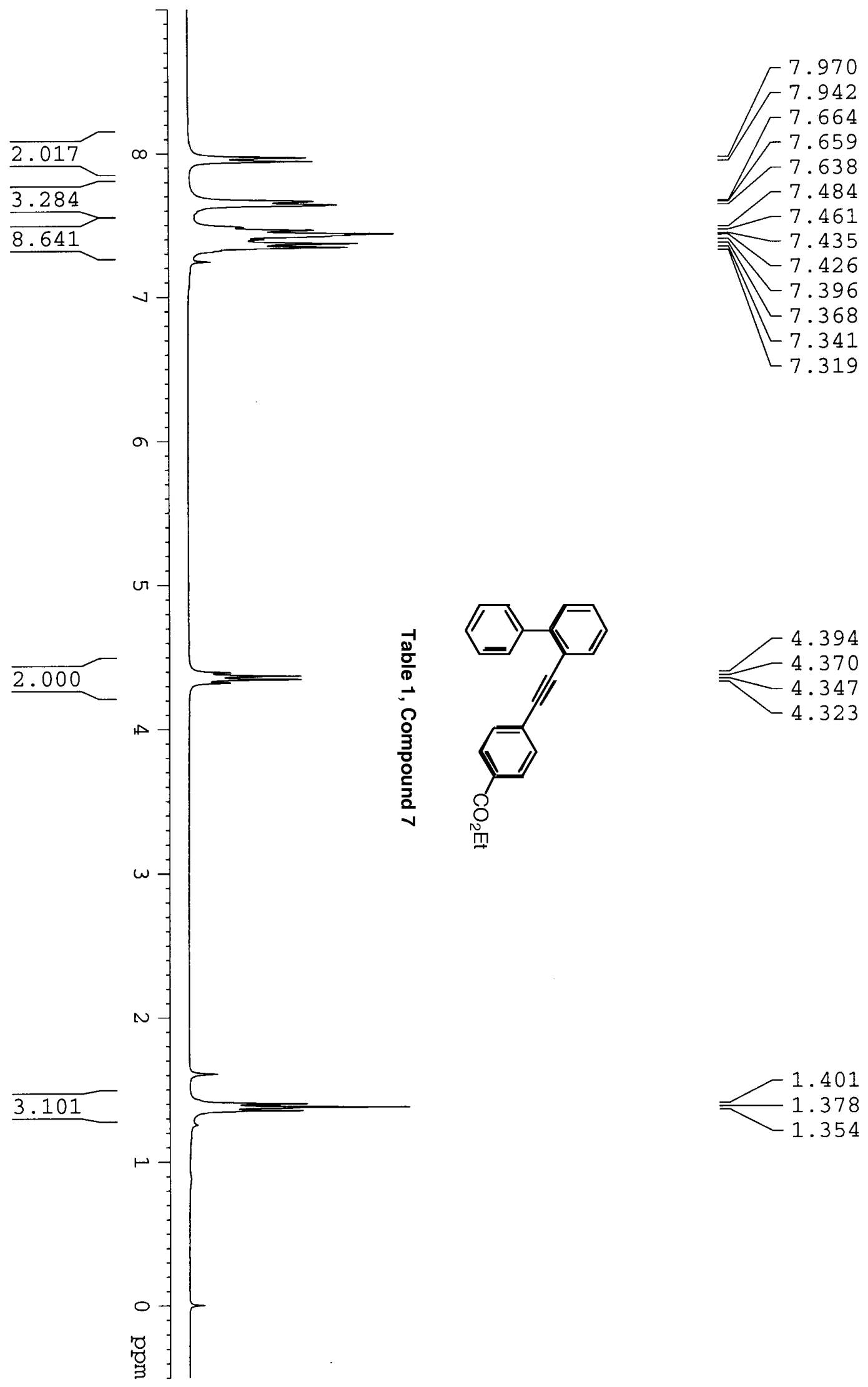

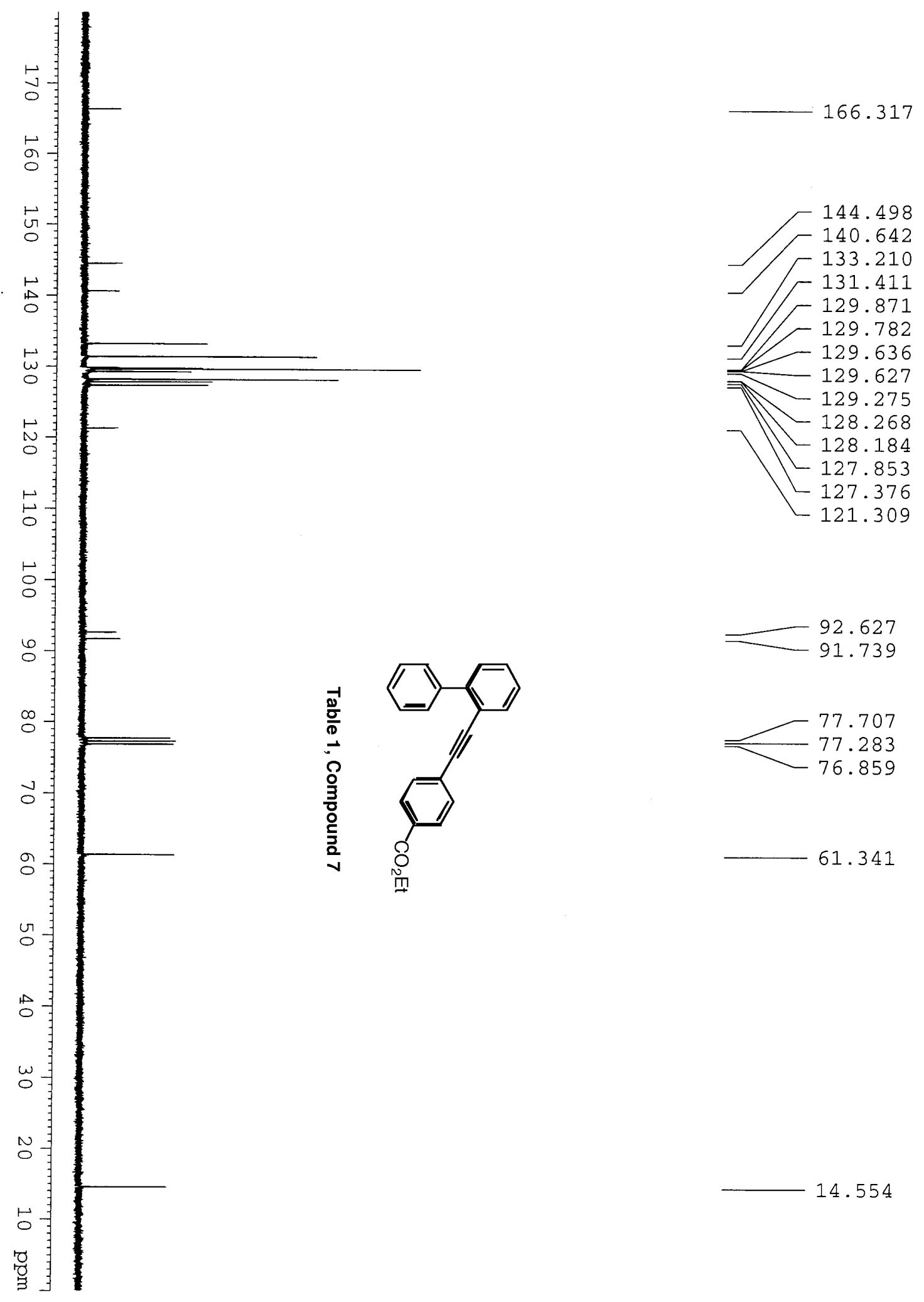

61.341

14.554 

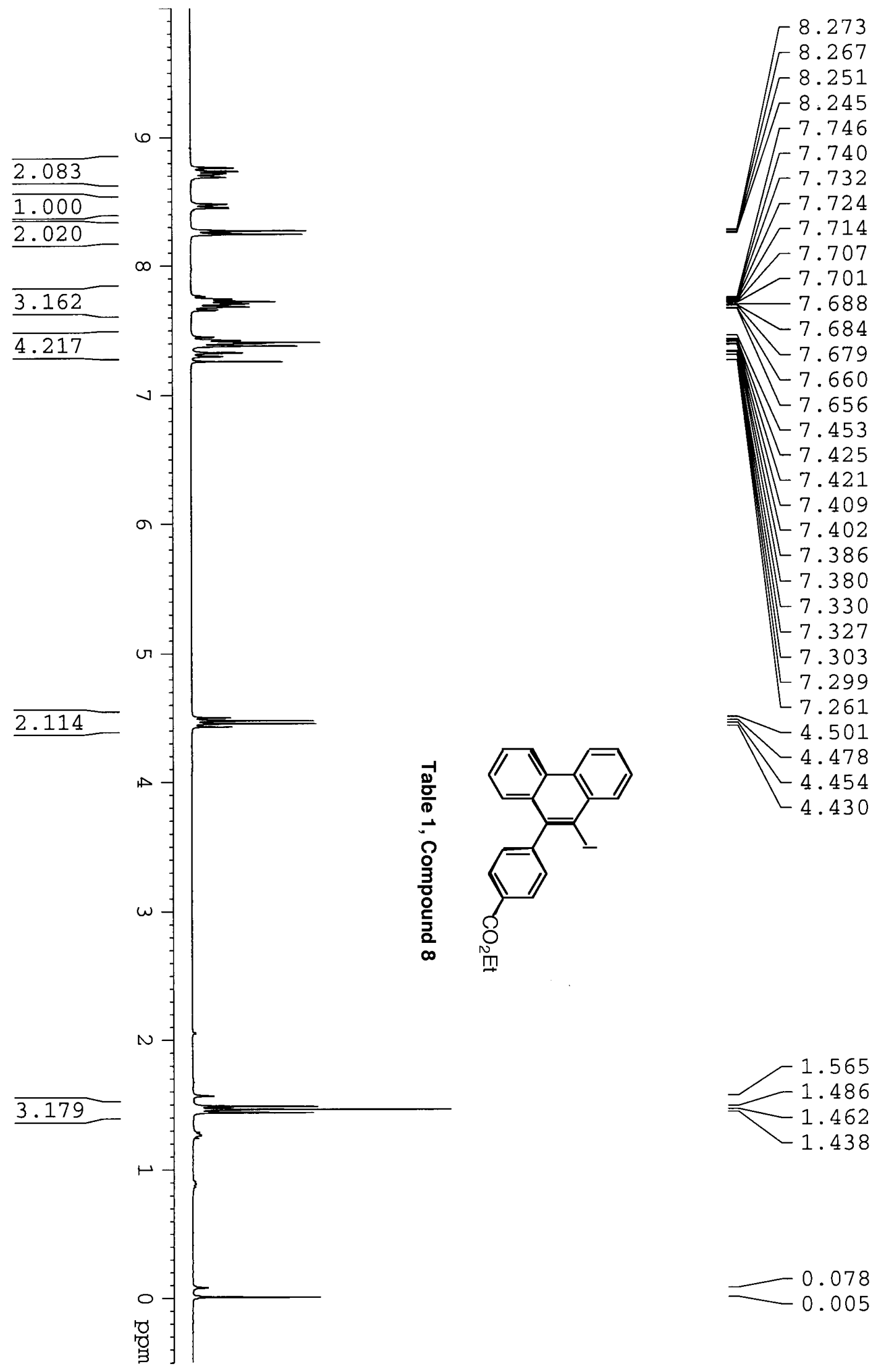

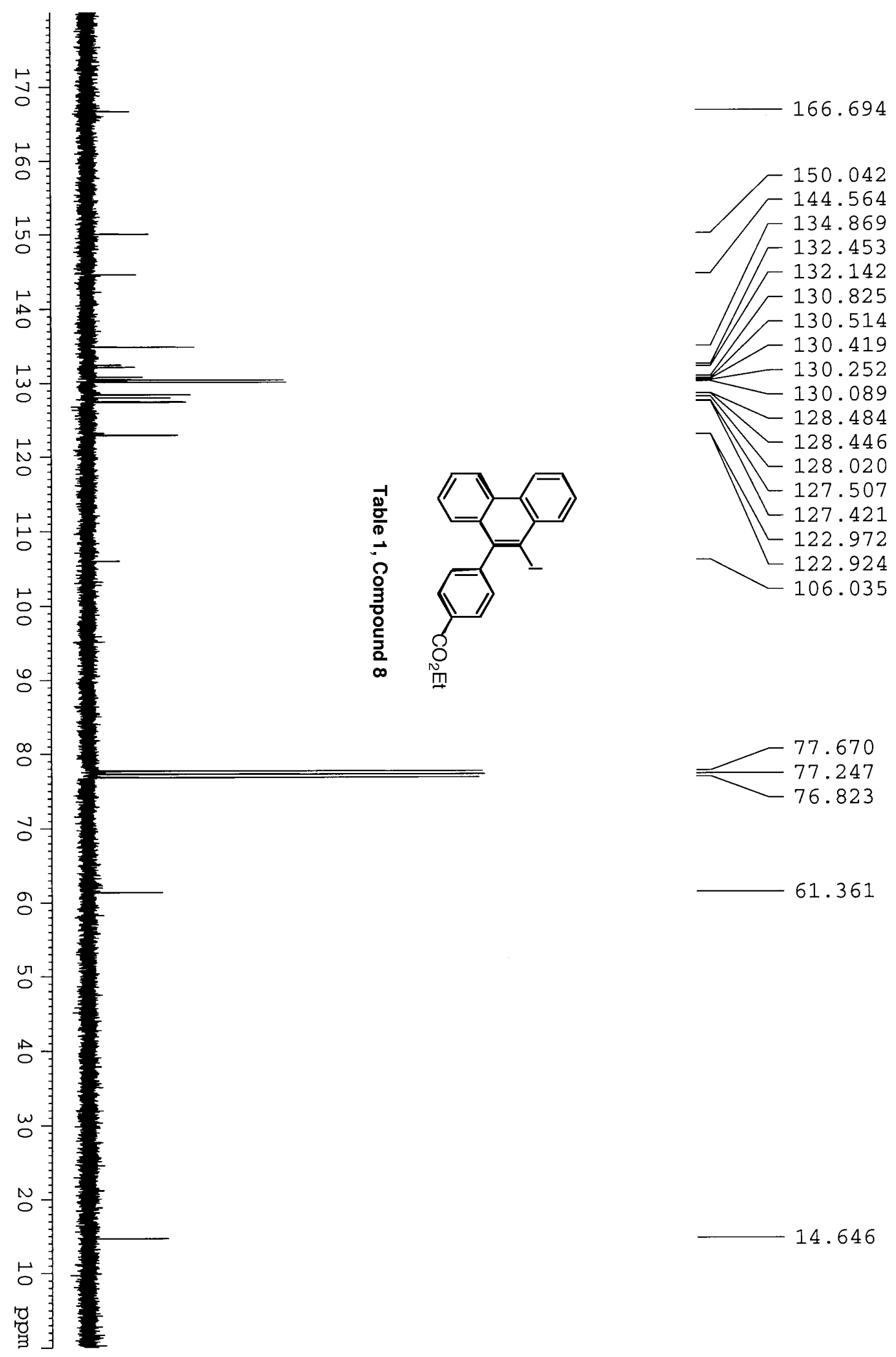

61.361 

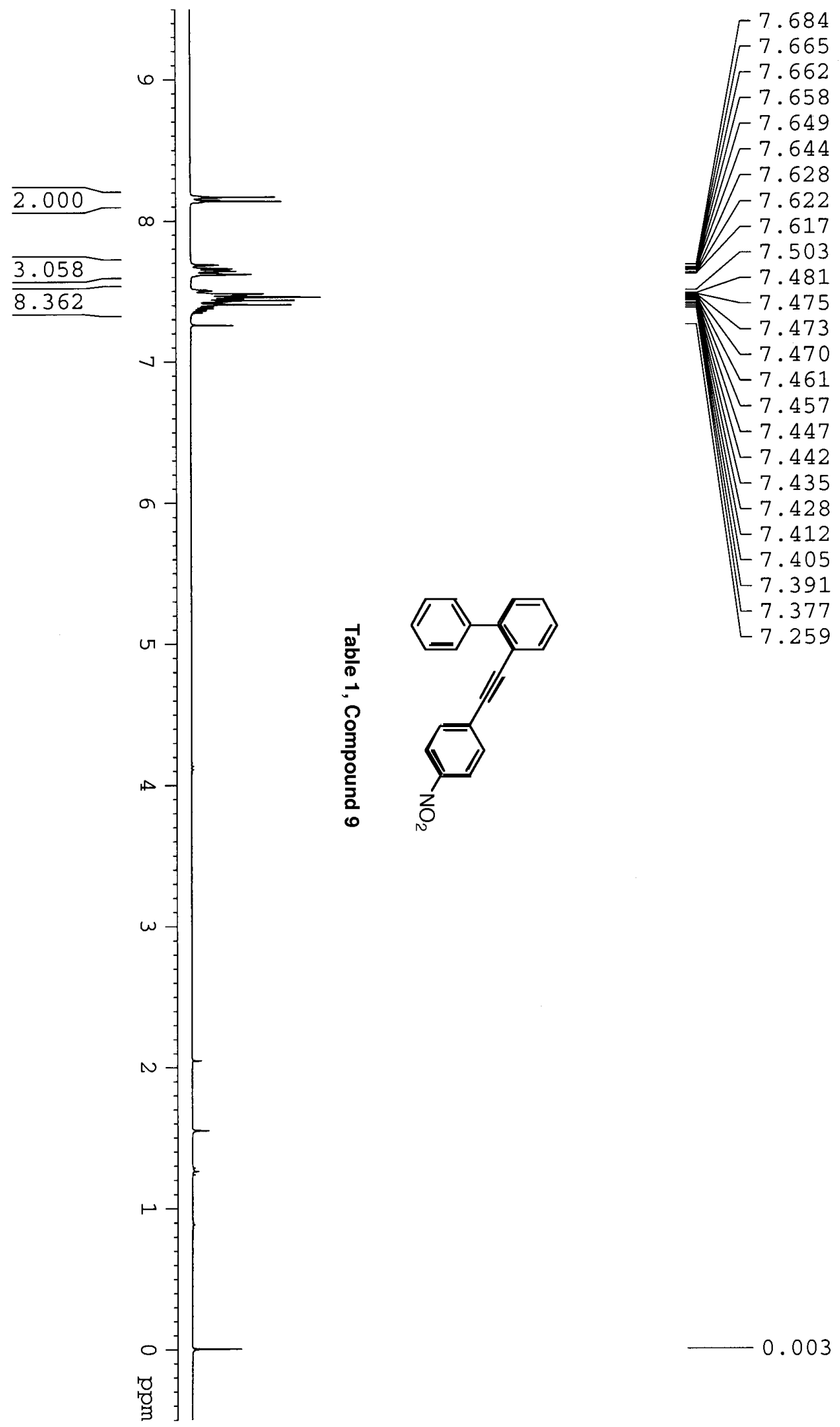


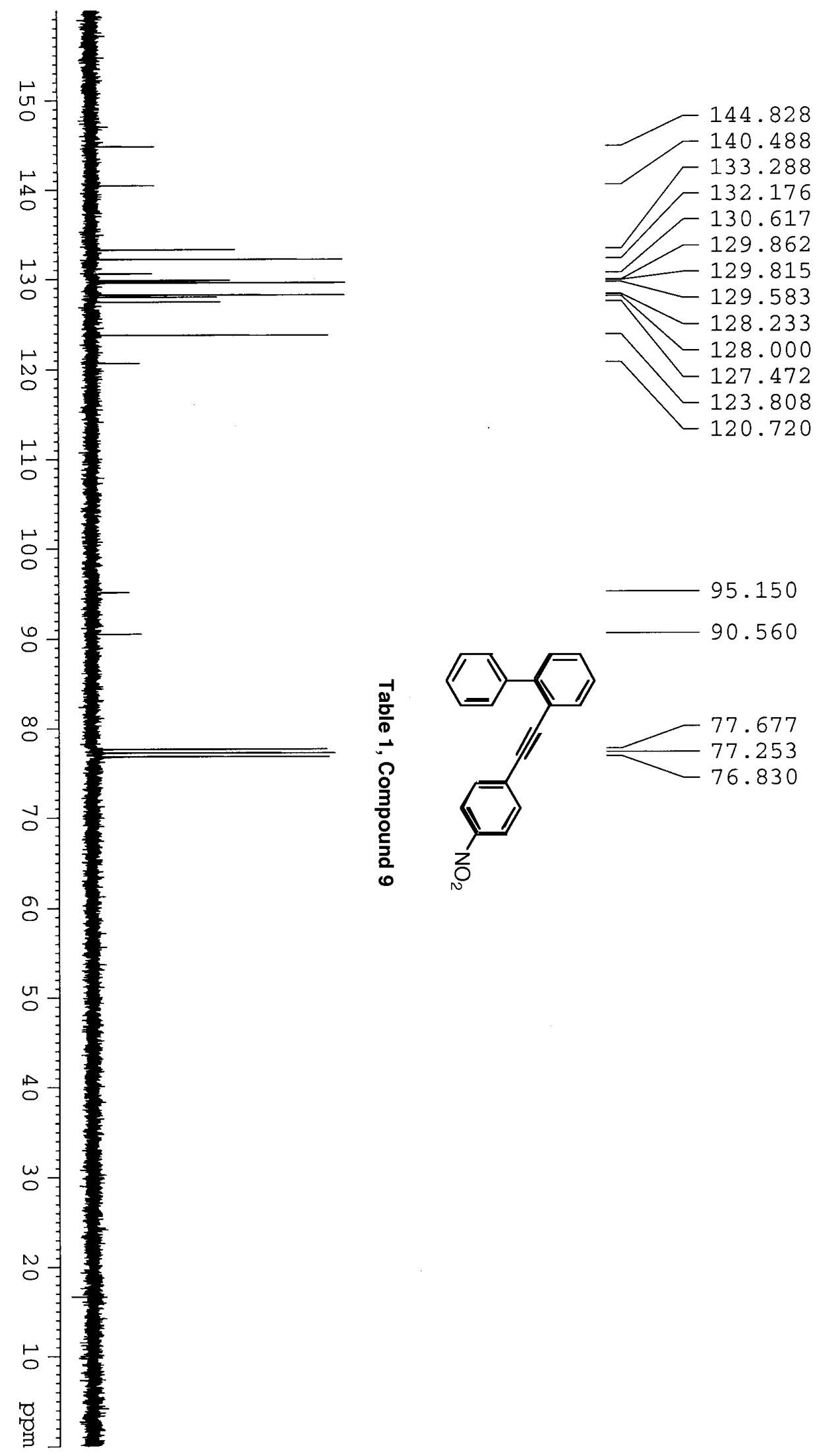



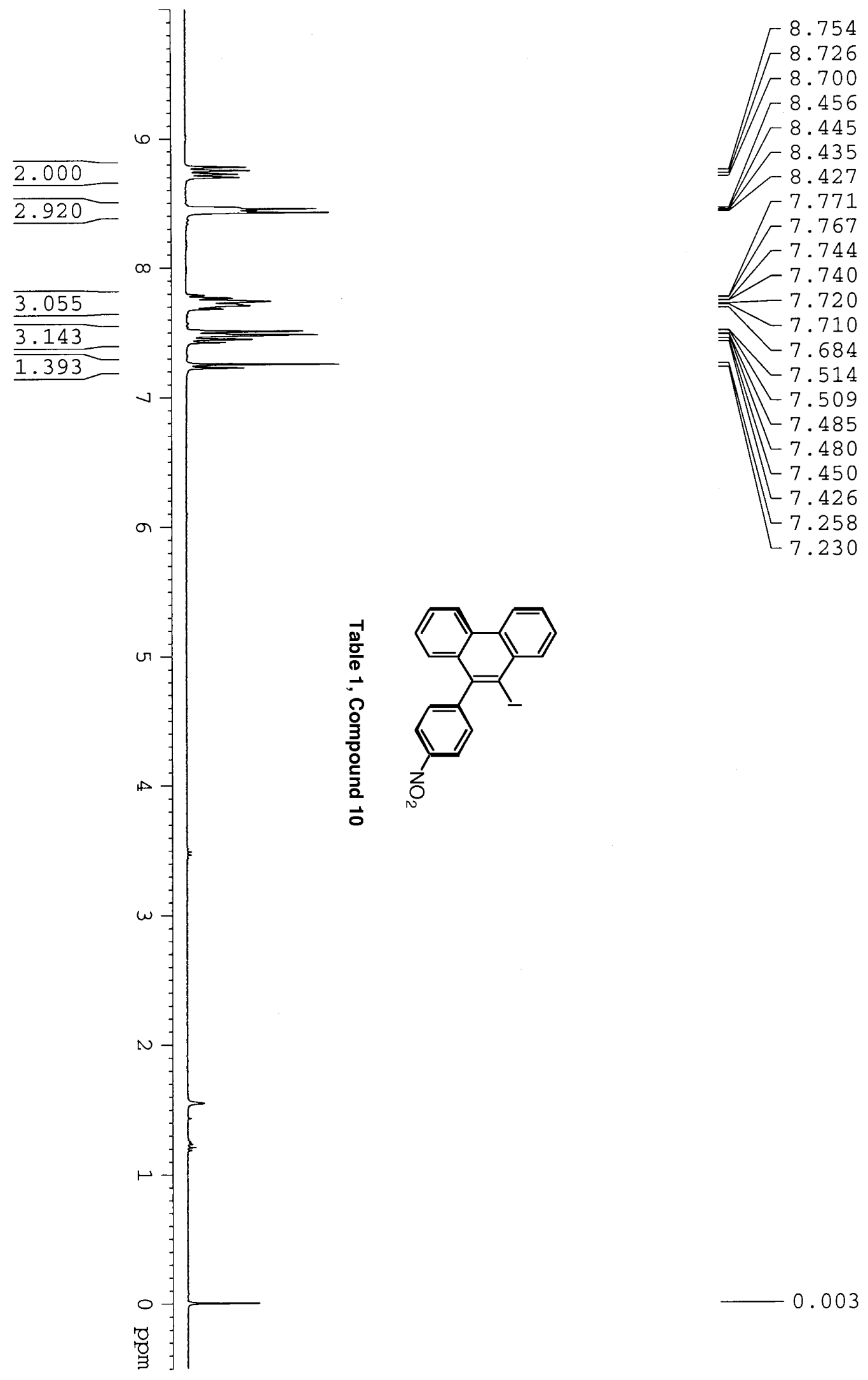

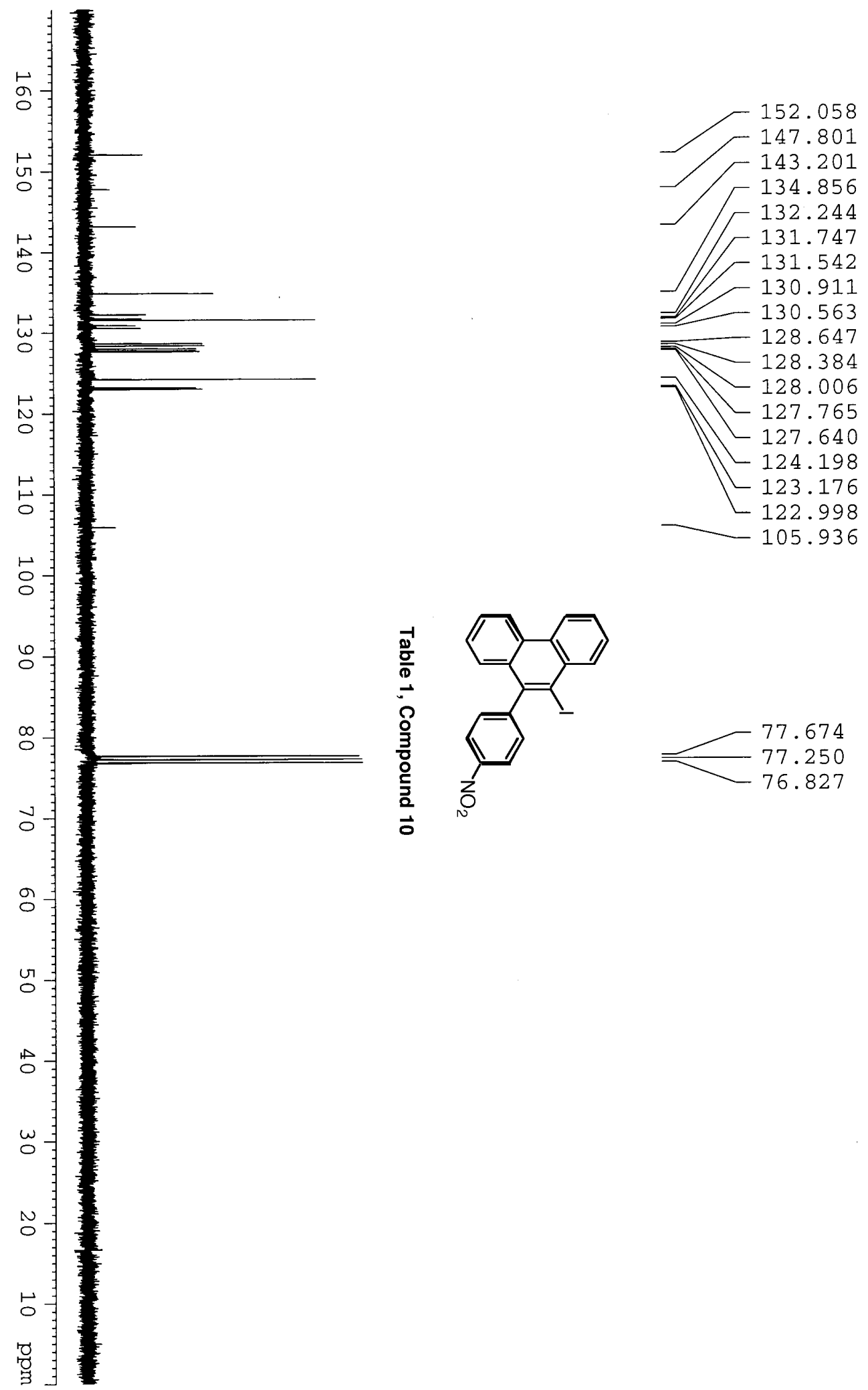

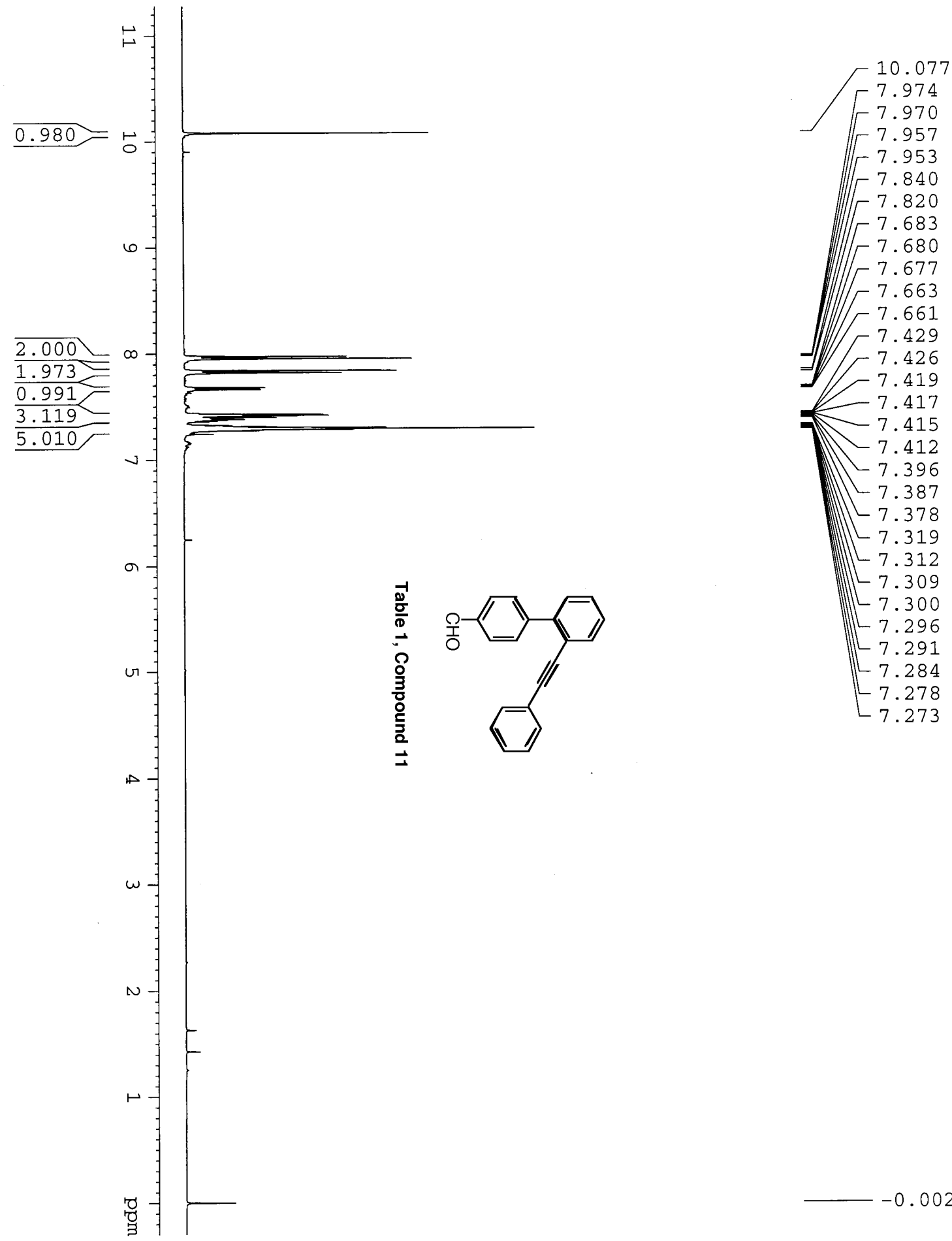


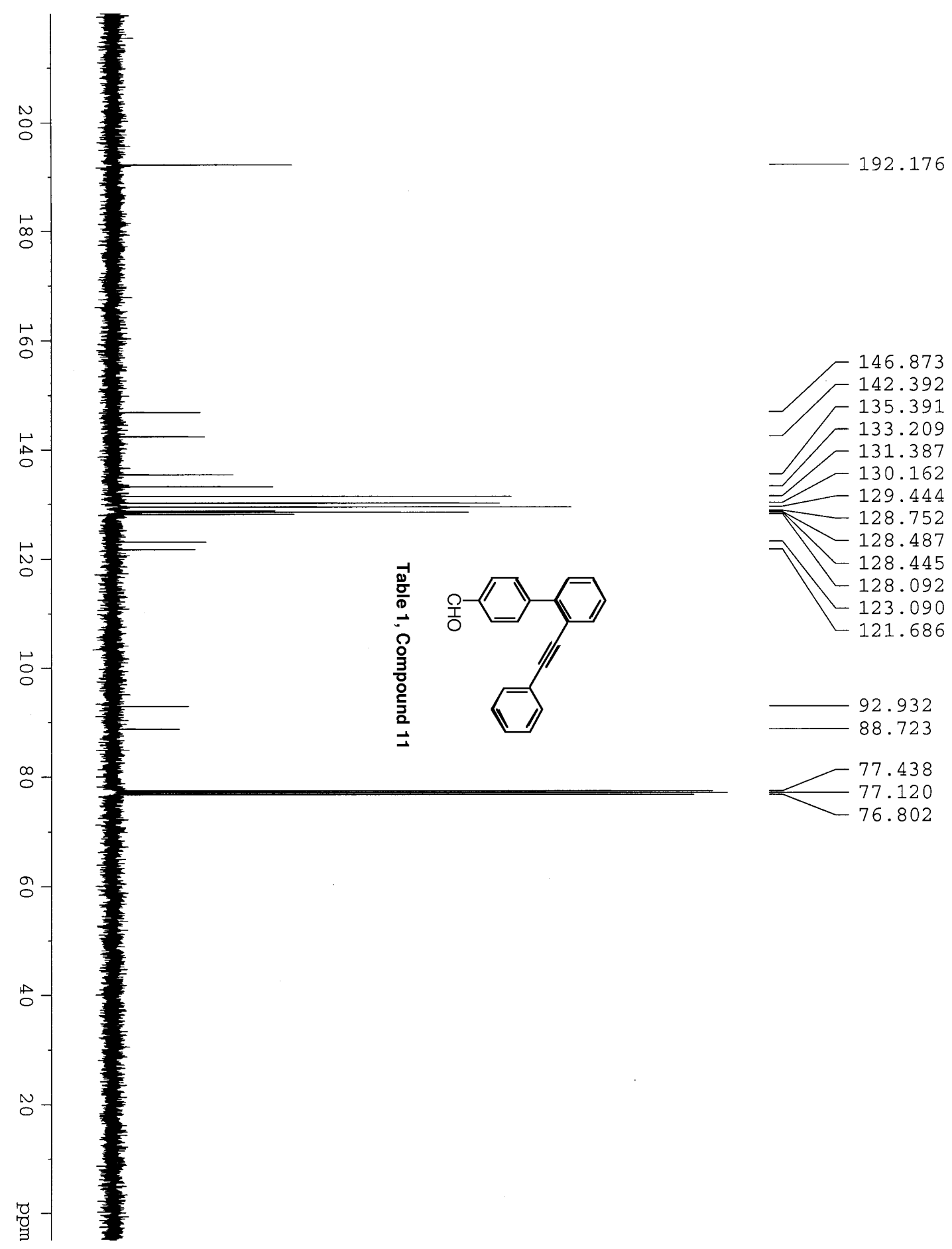



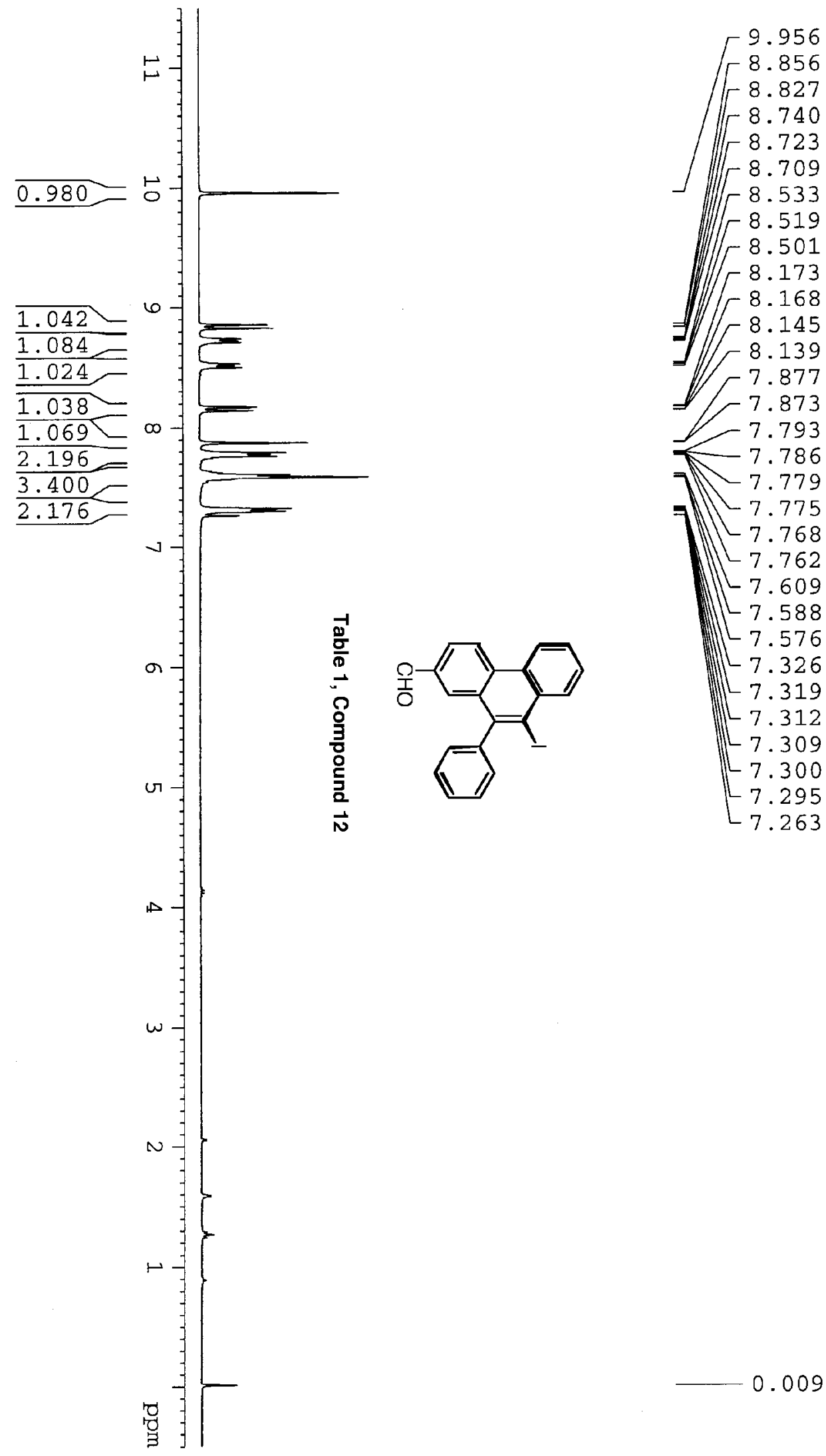


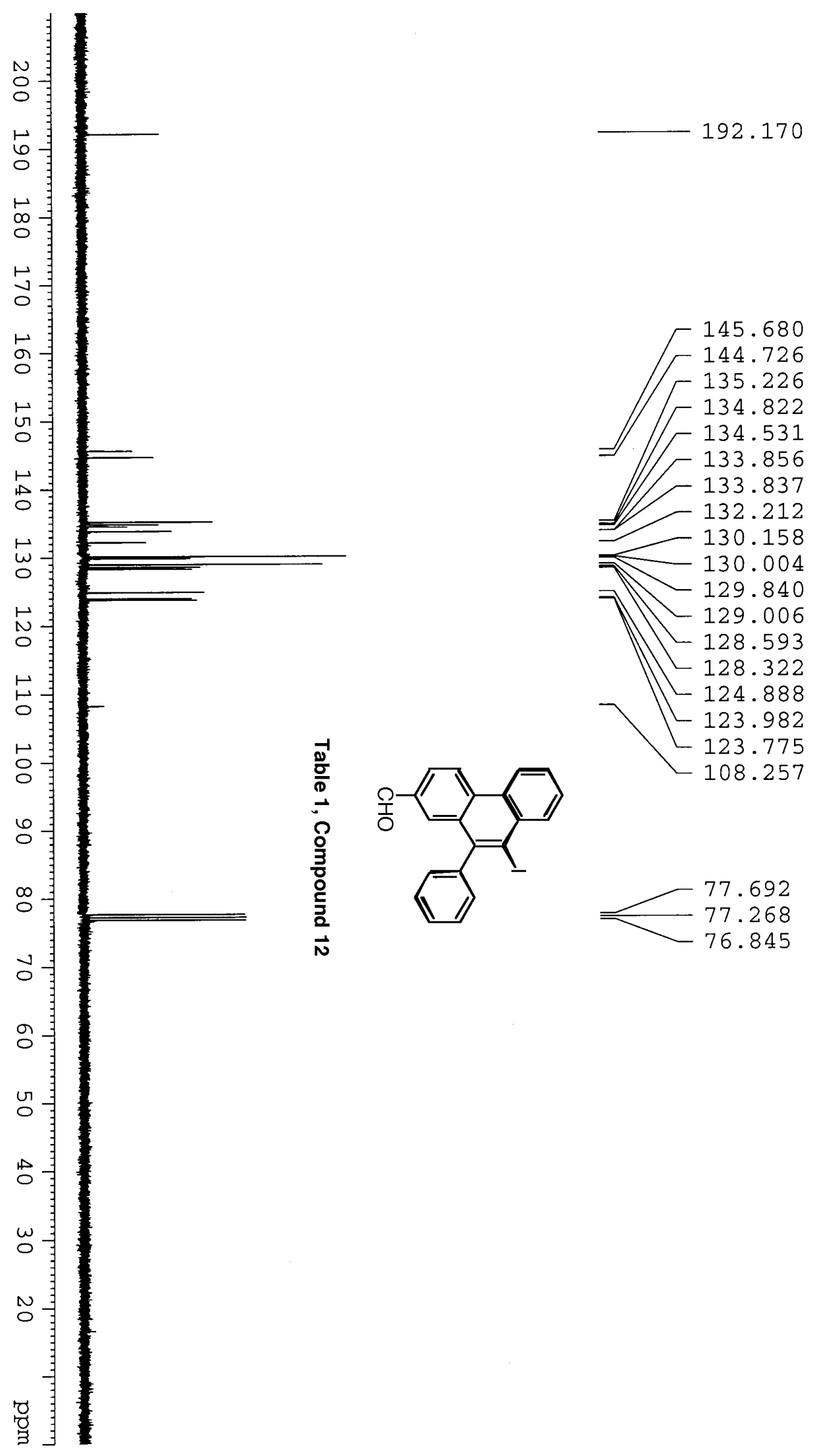




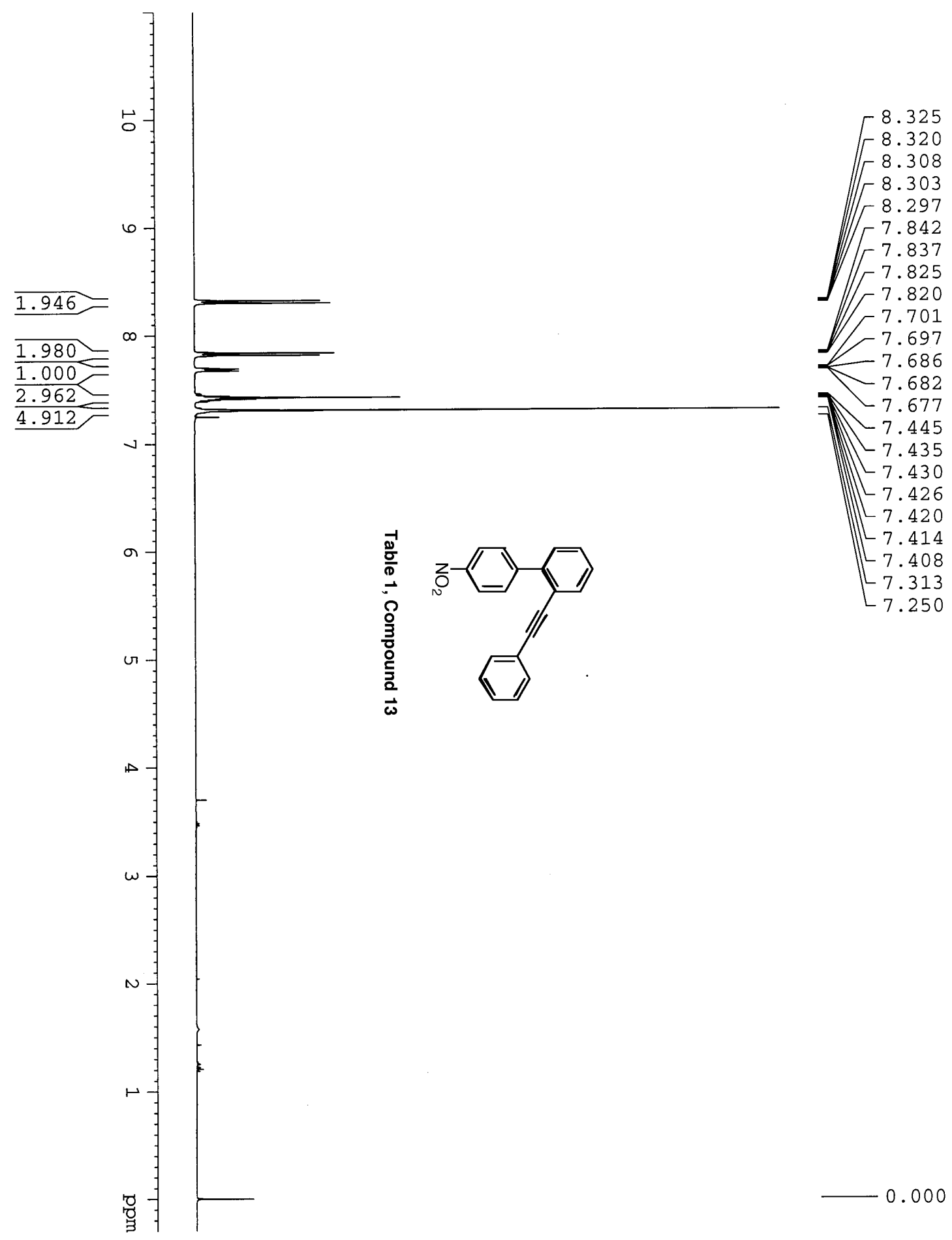




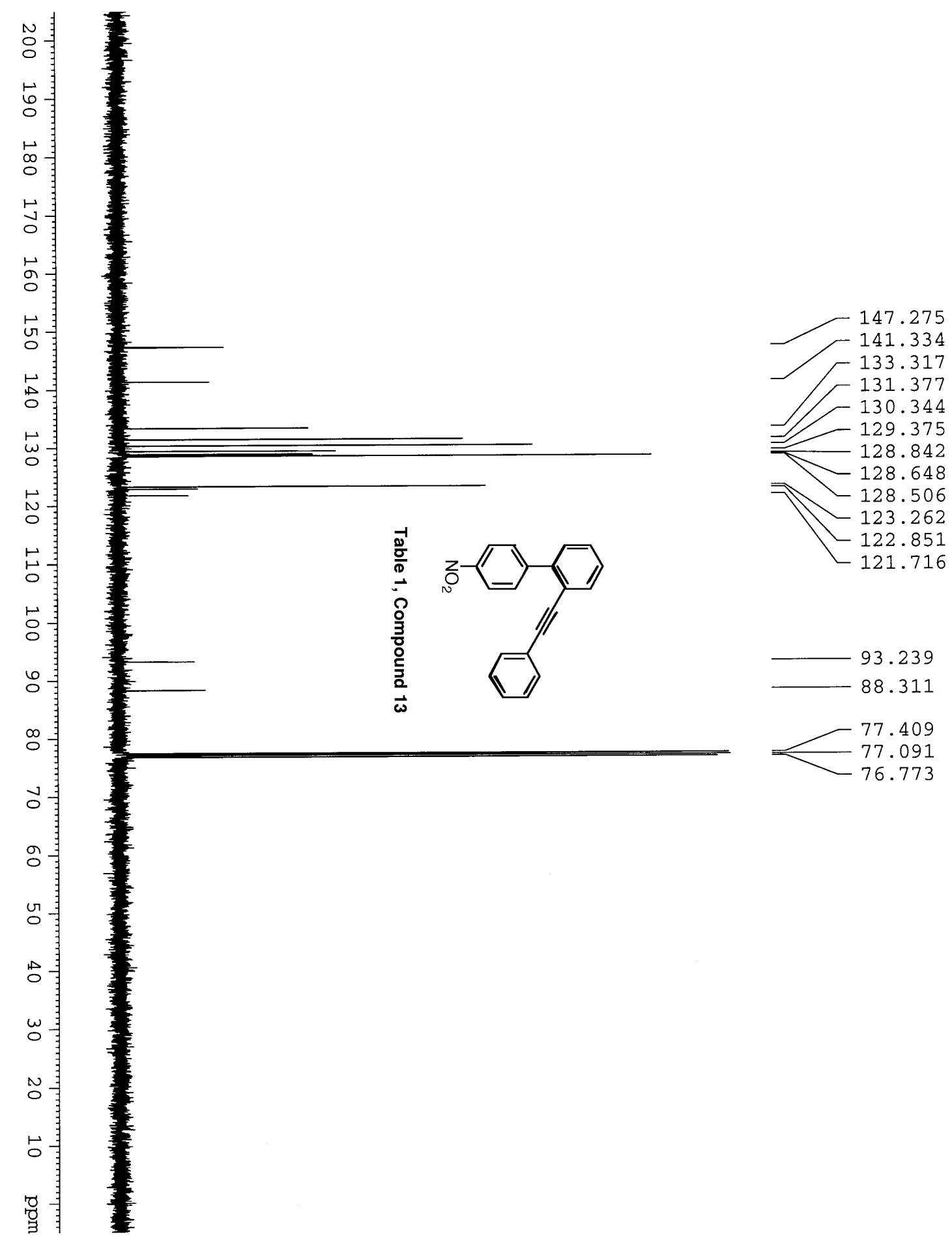



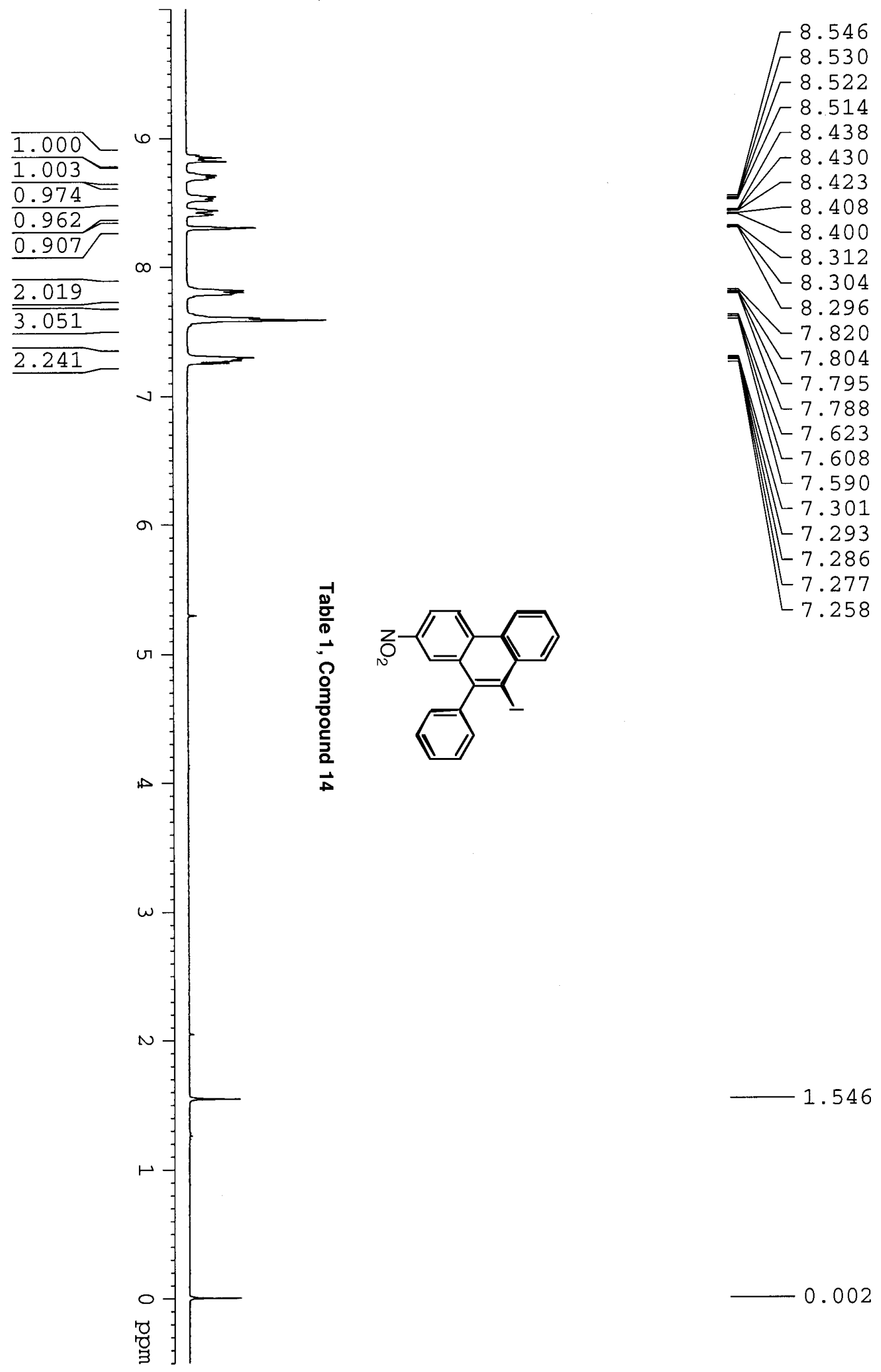


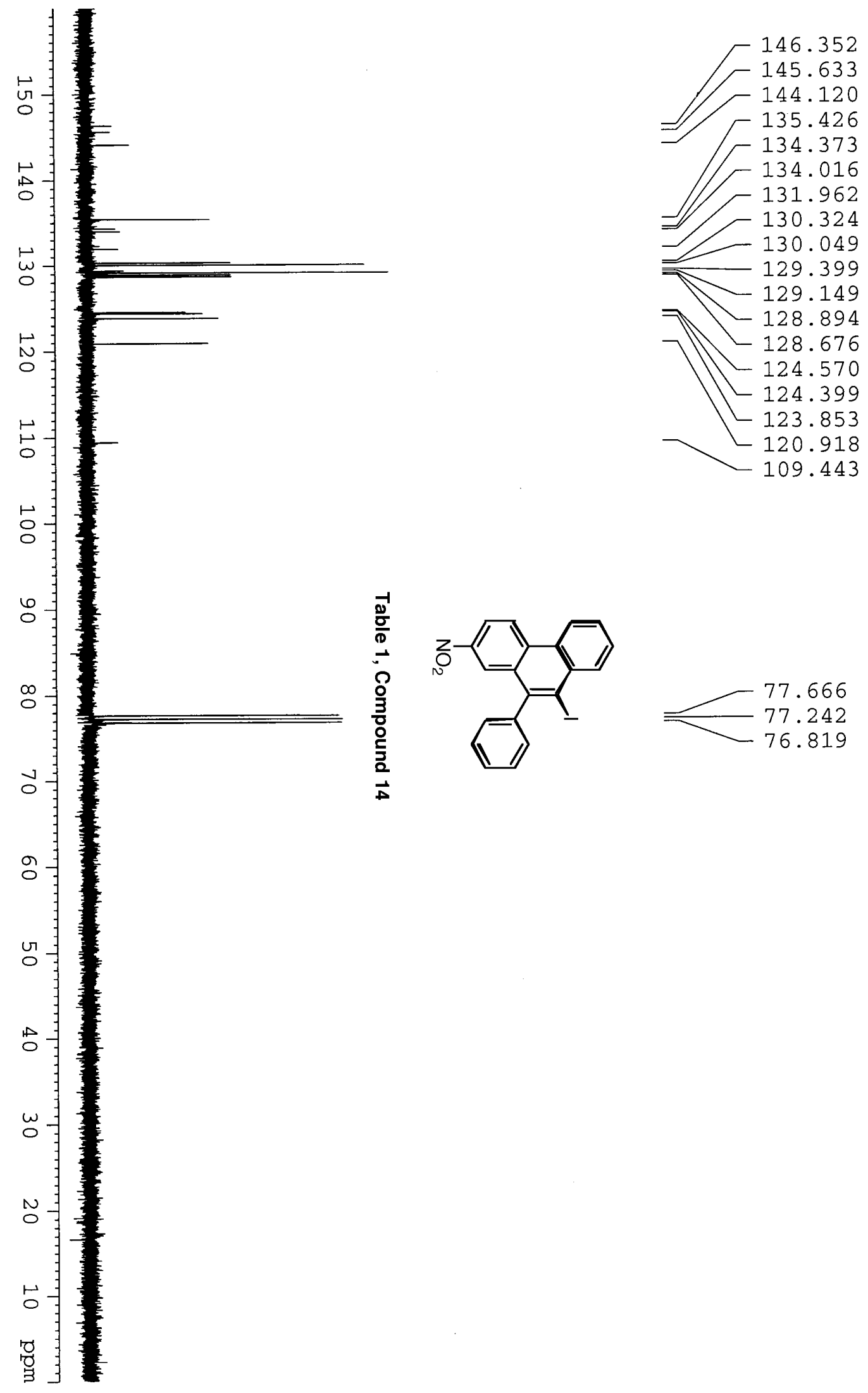




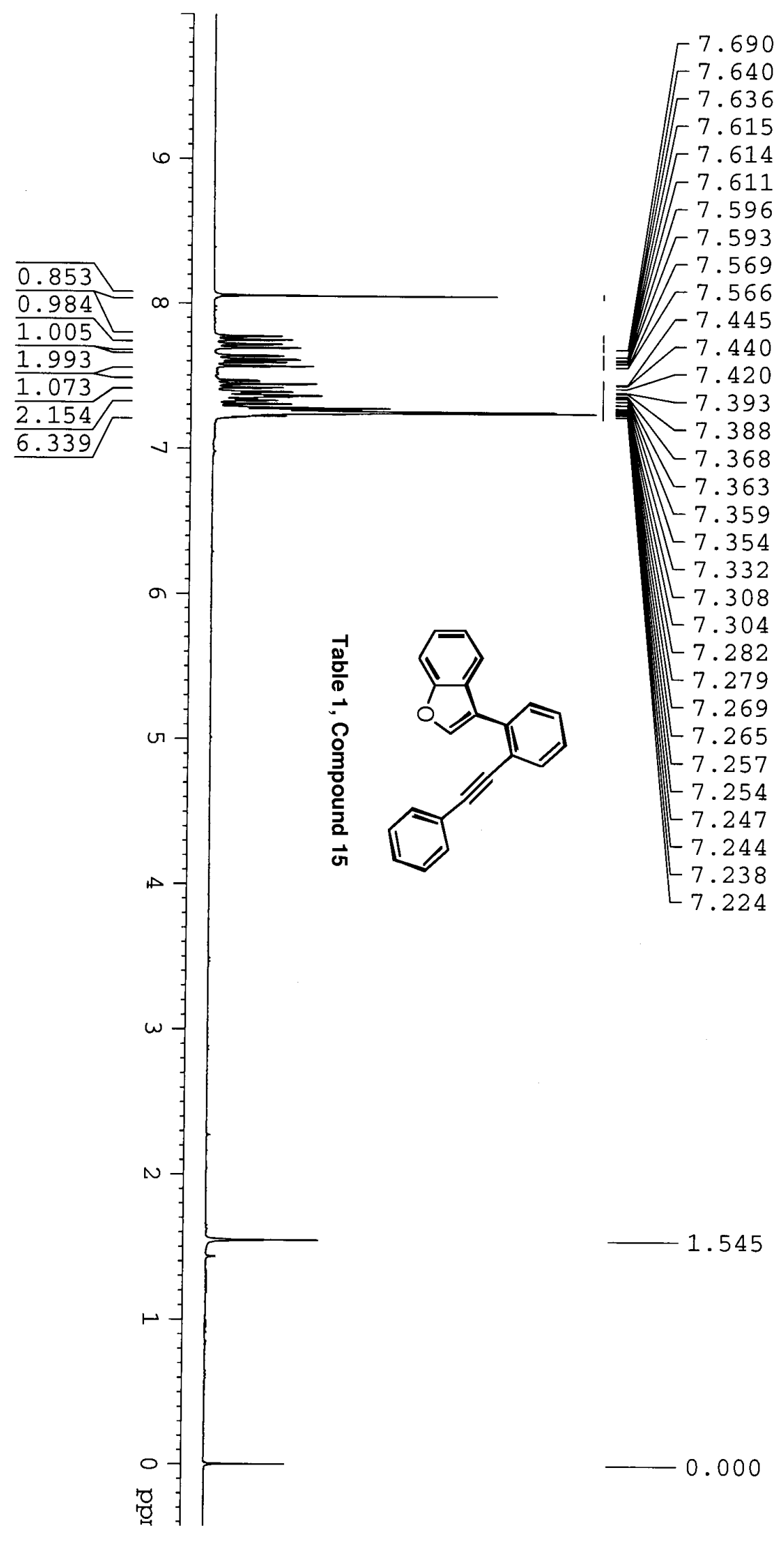




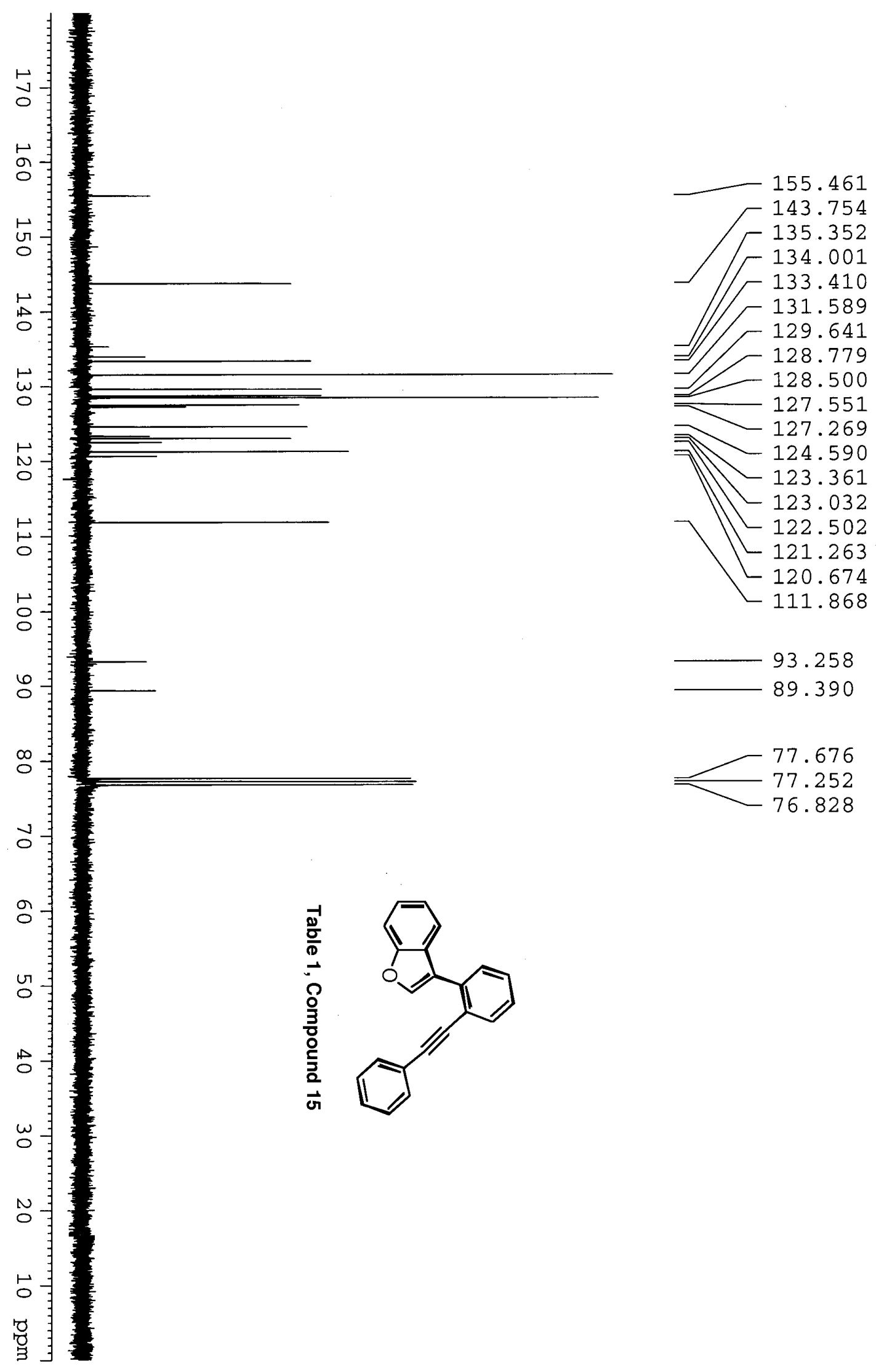



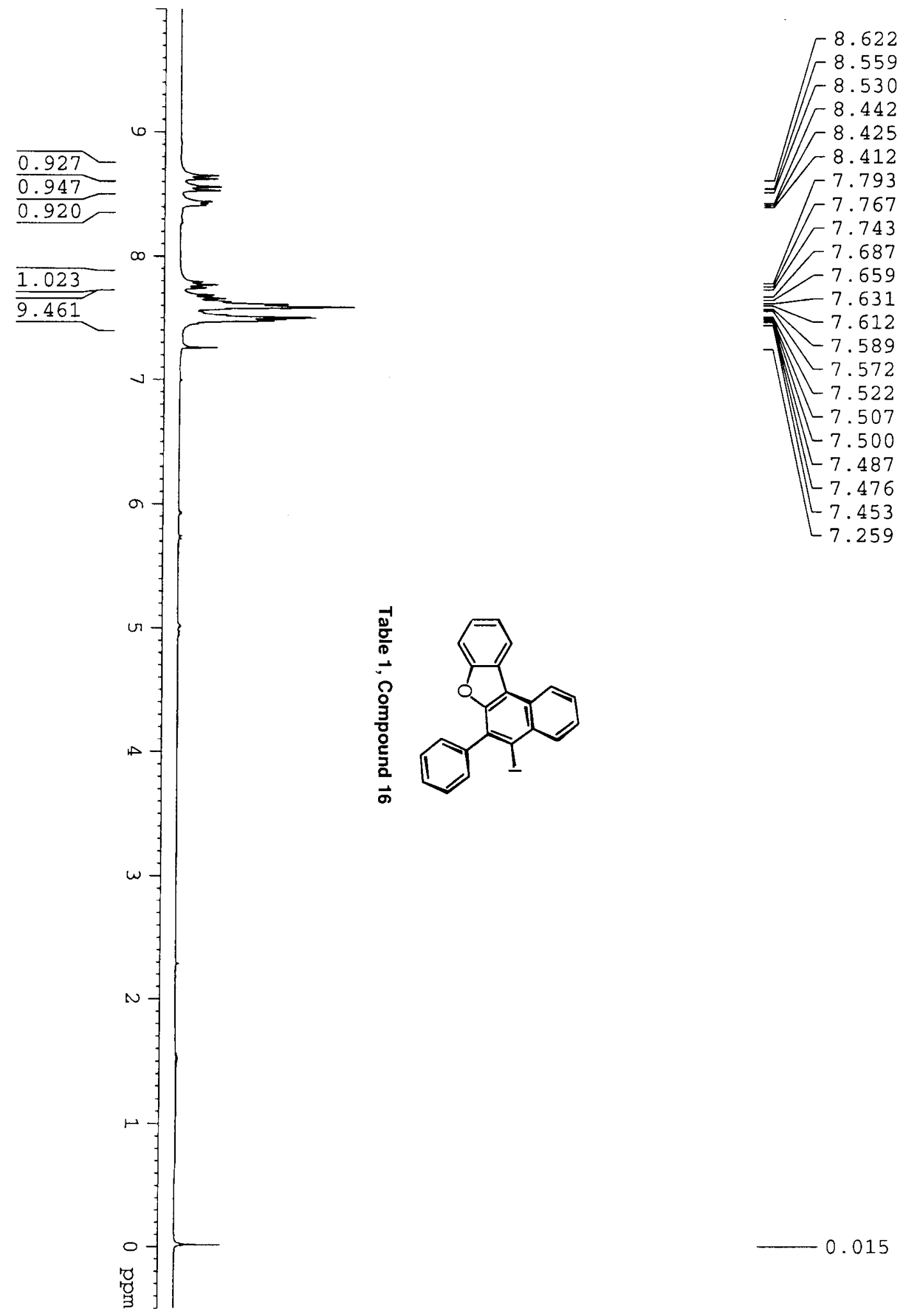


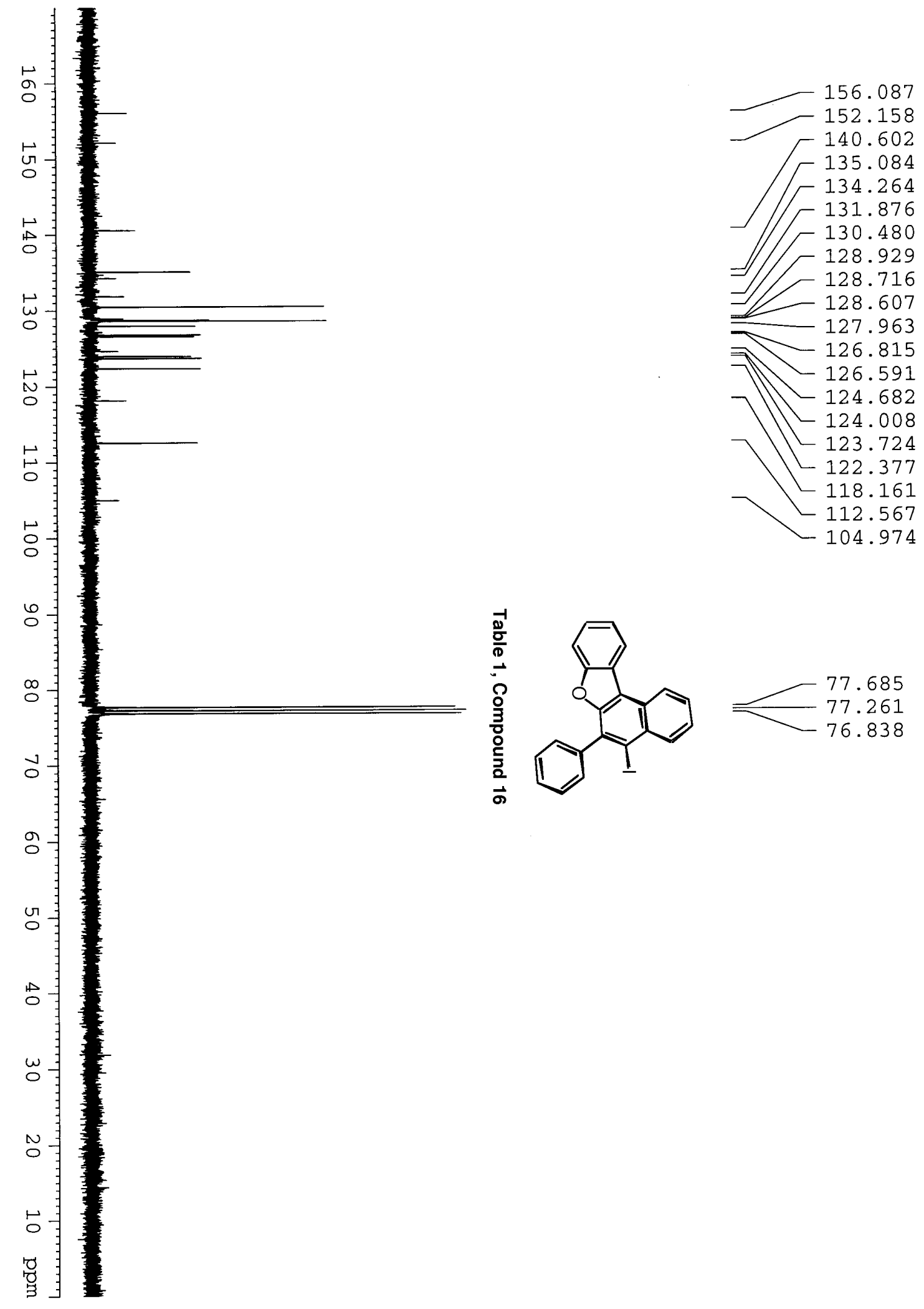



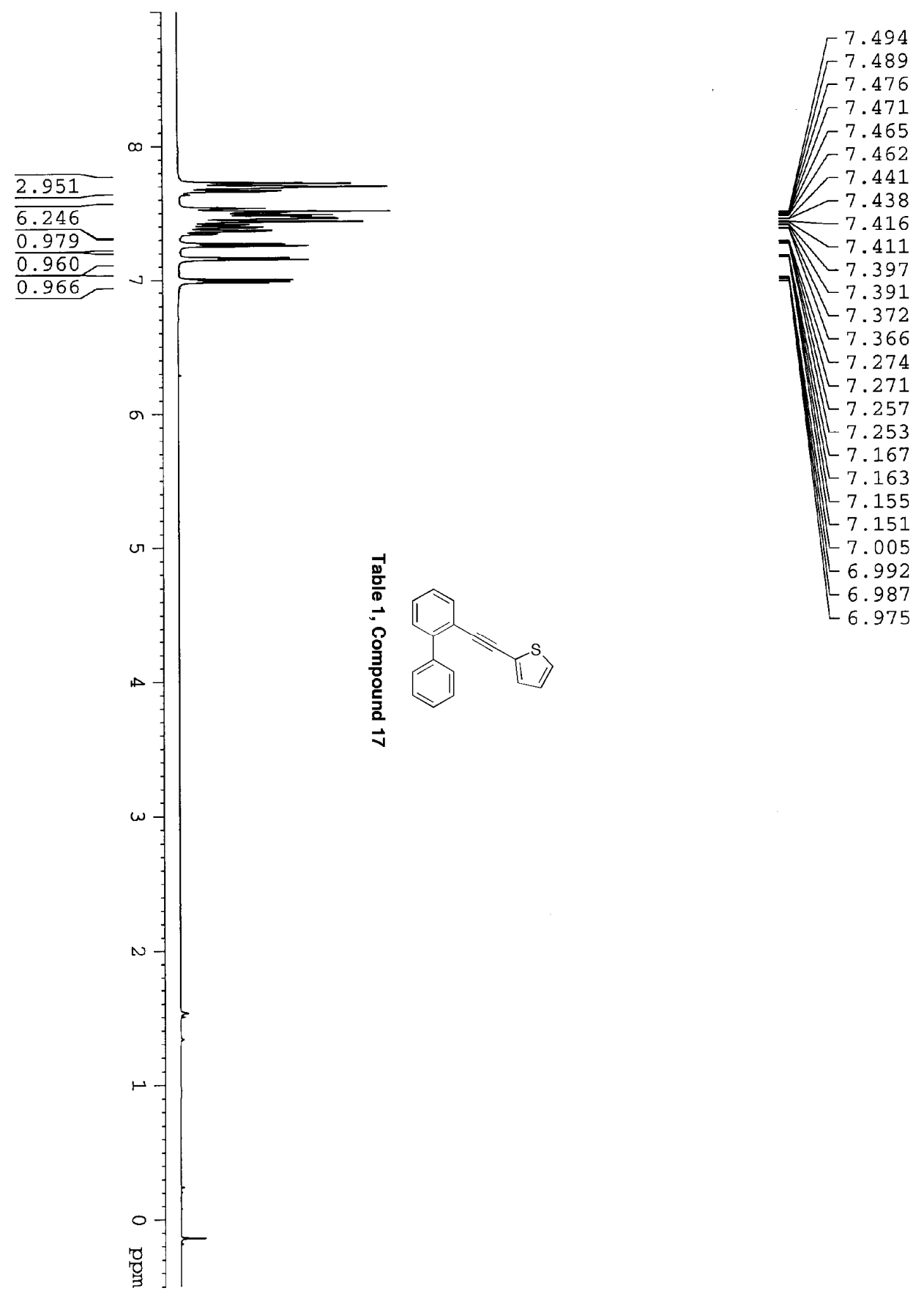


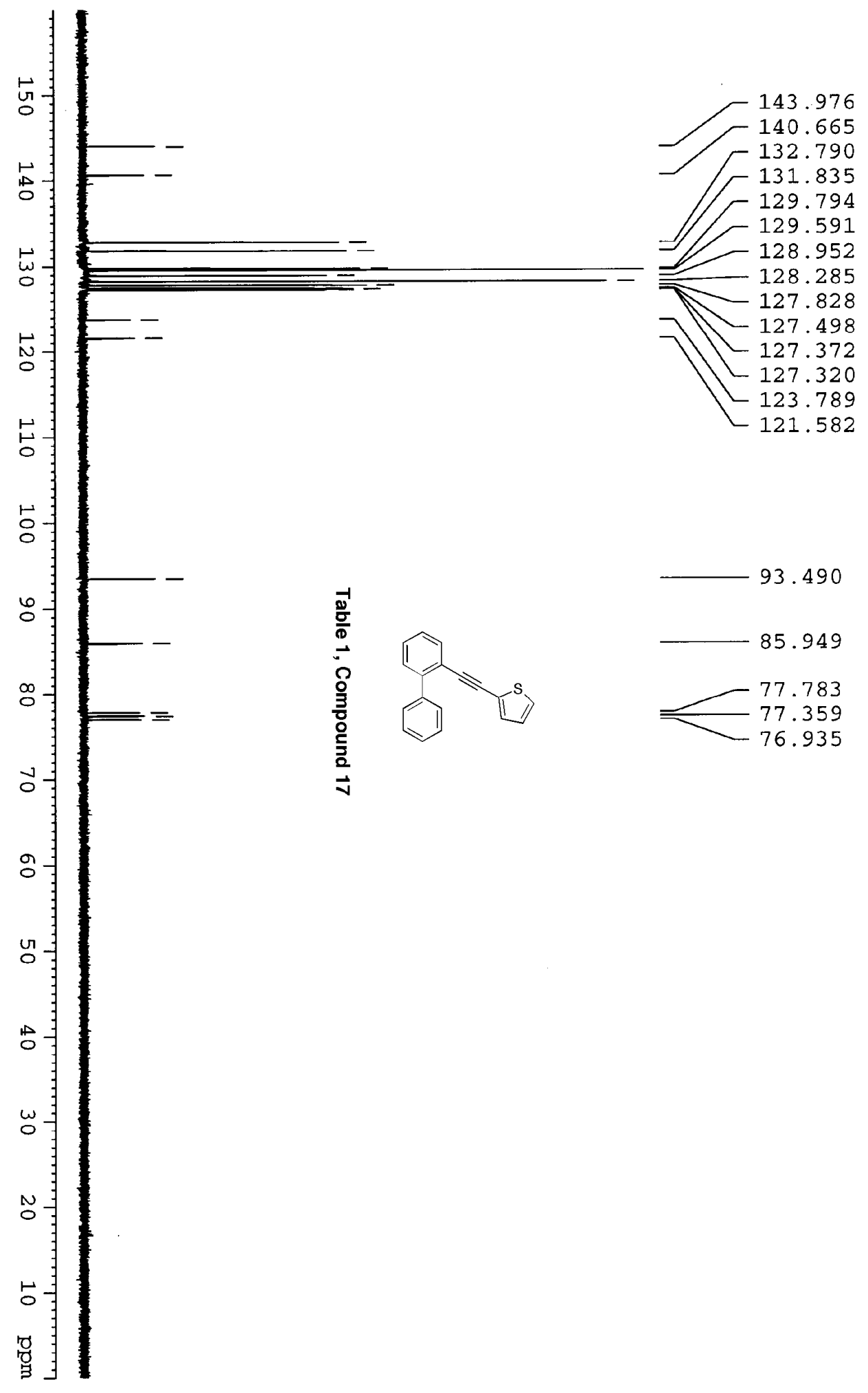




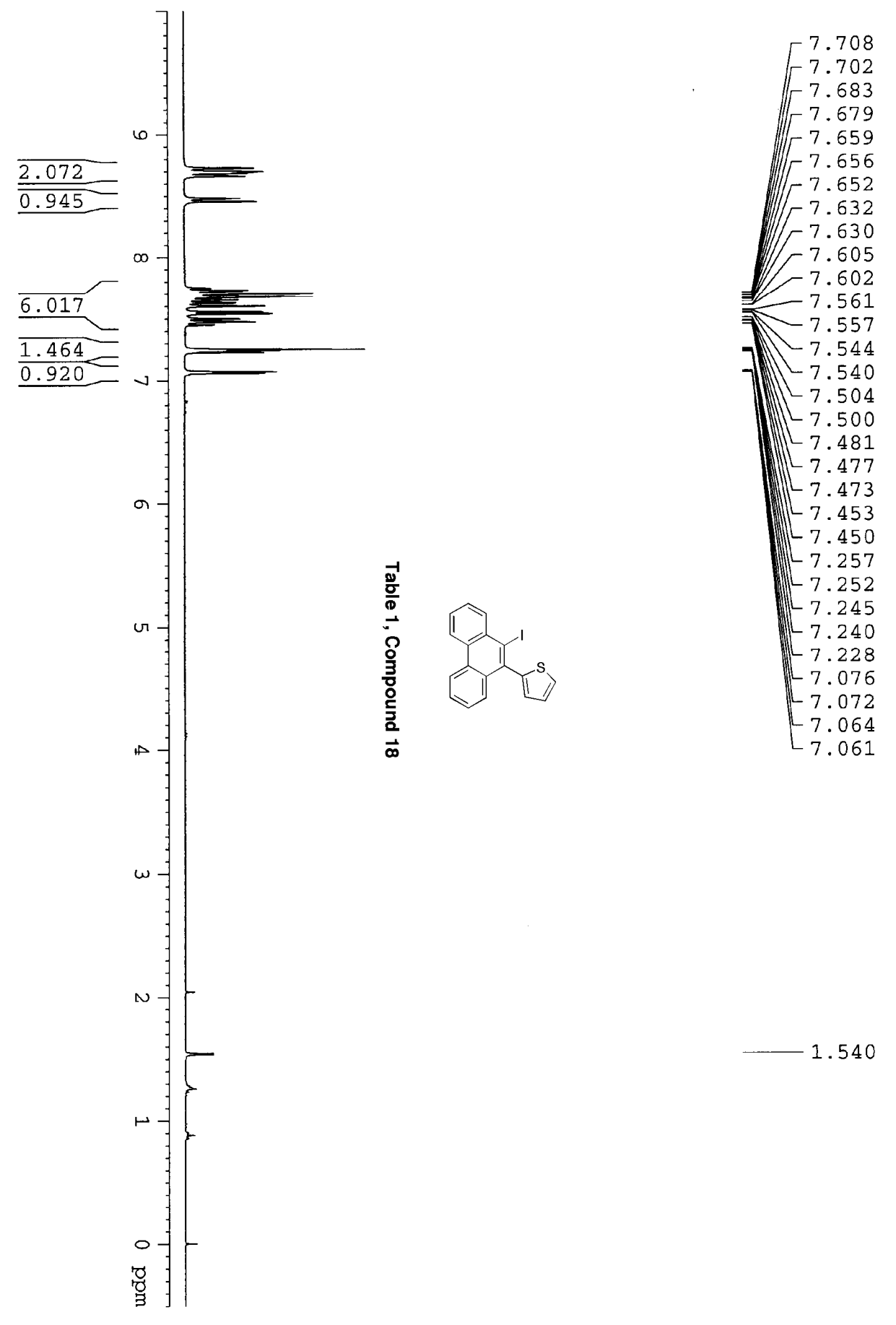




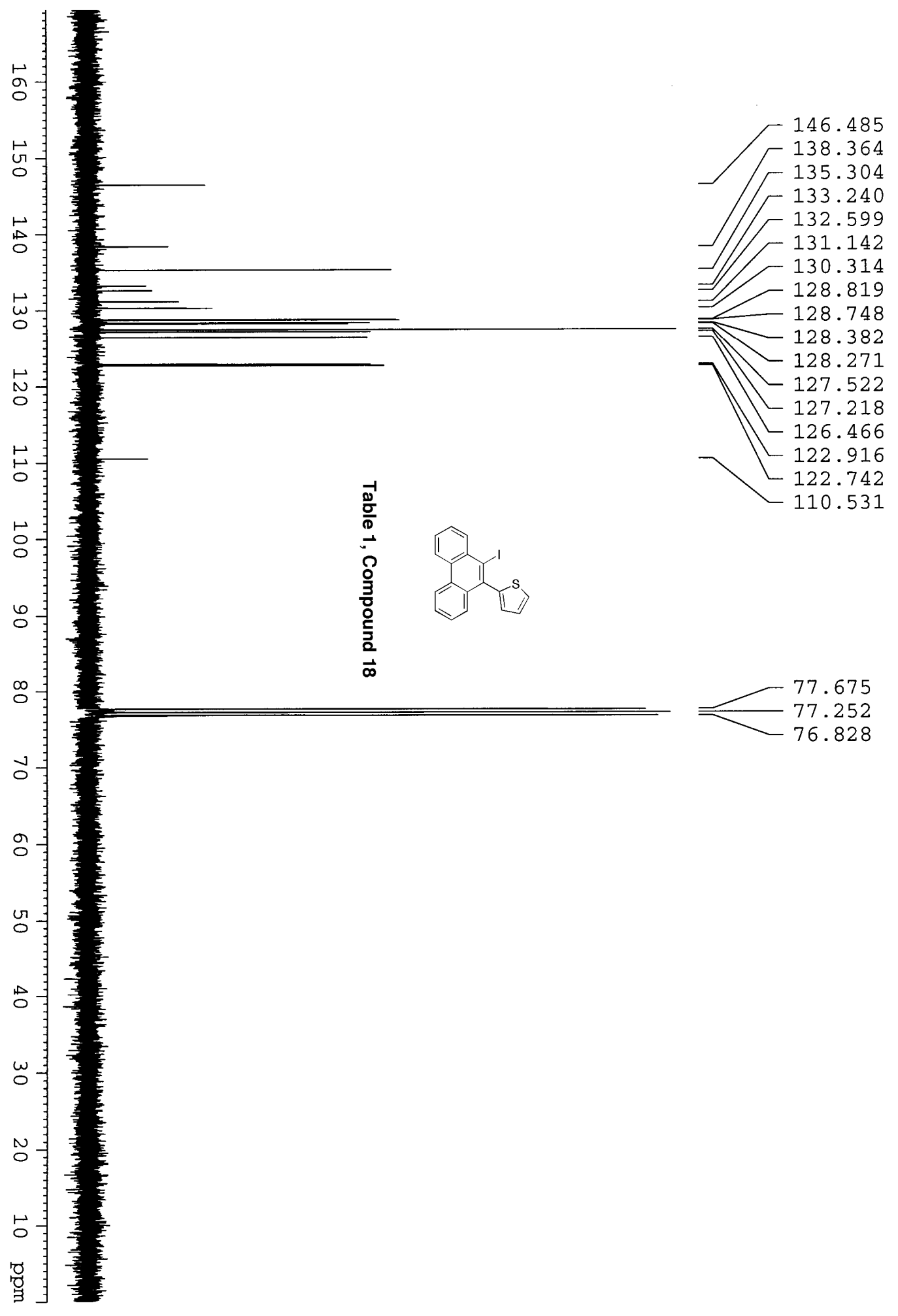



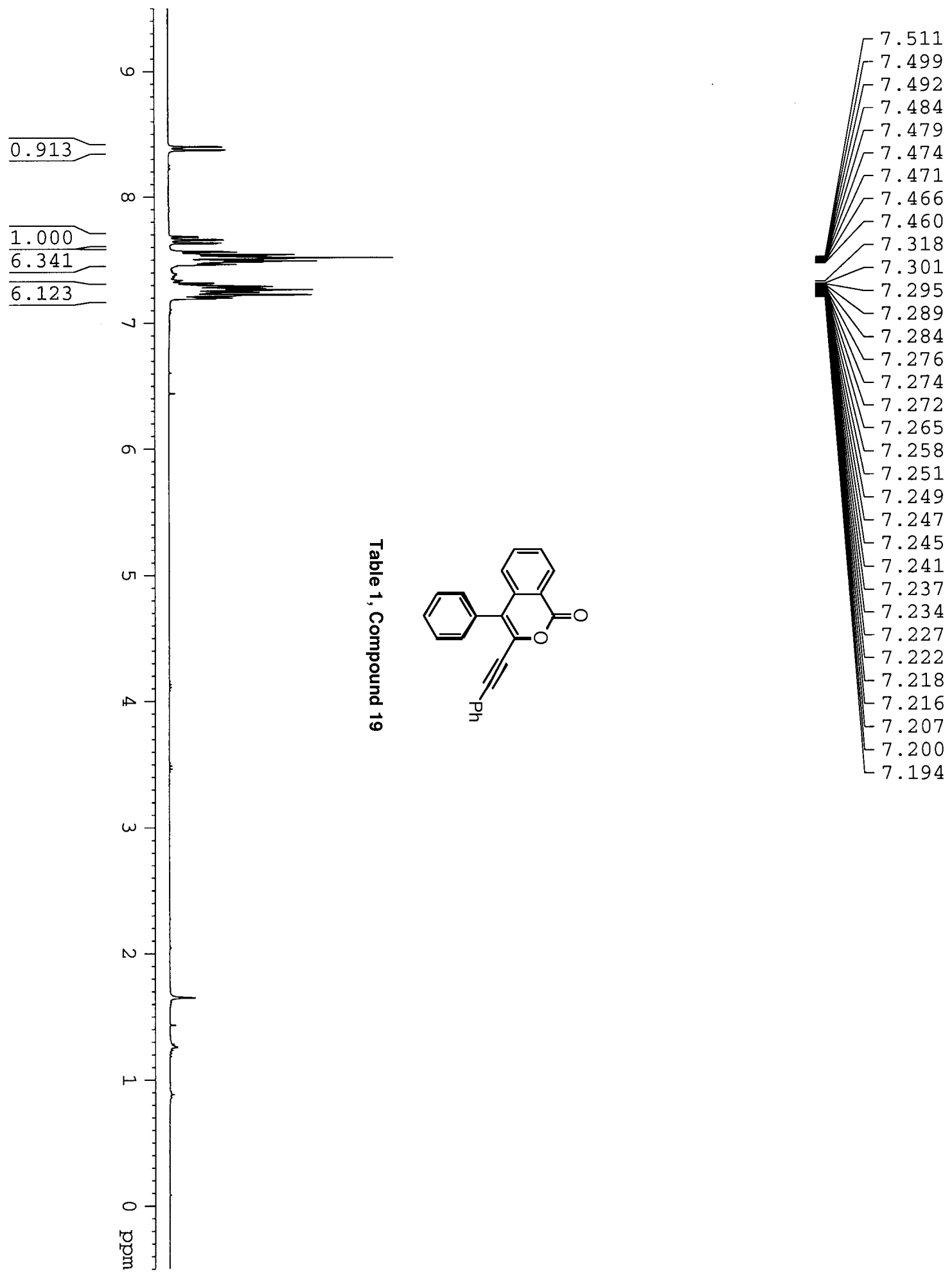


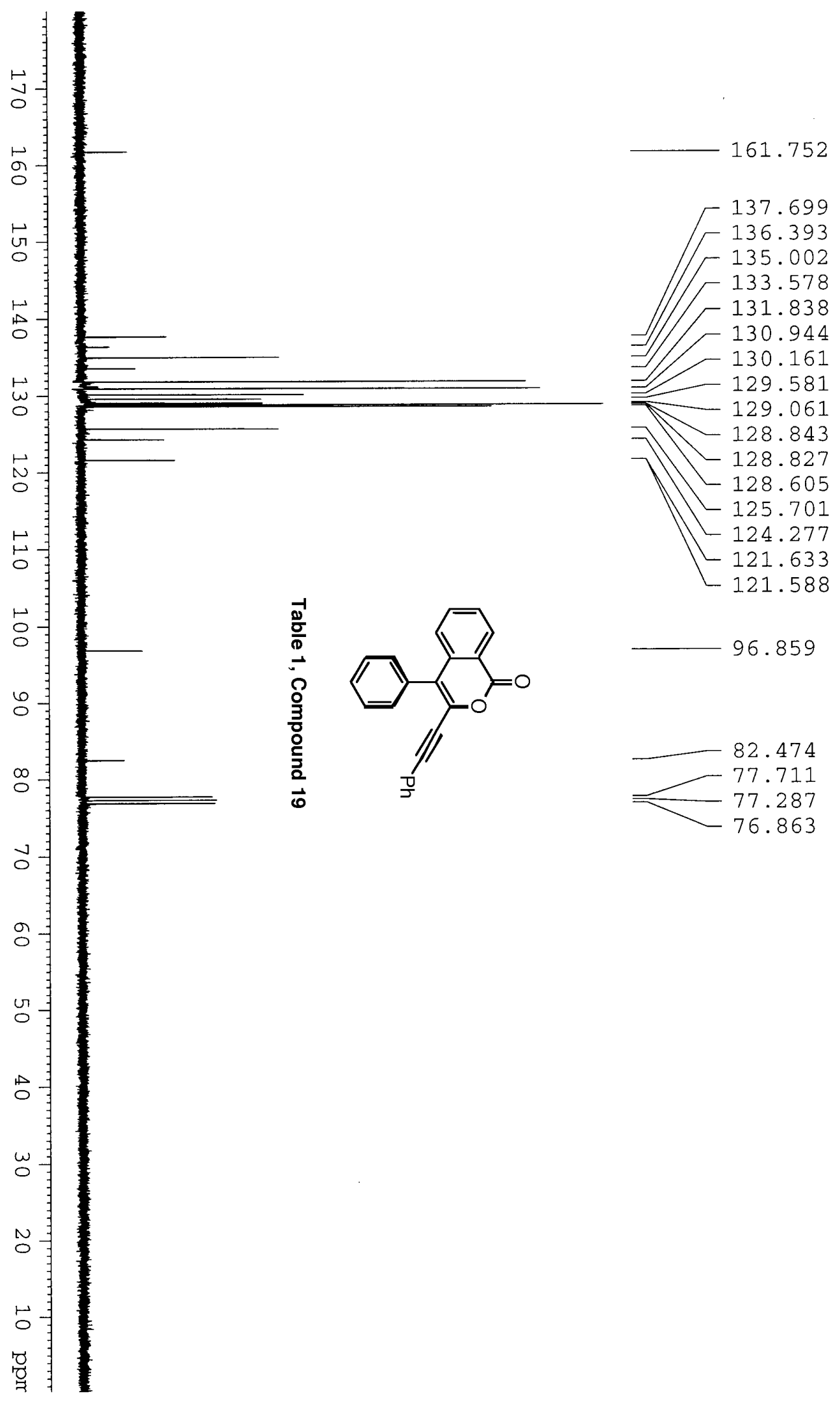




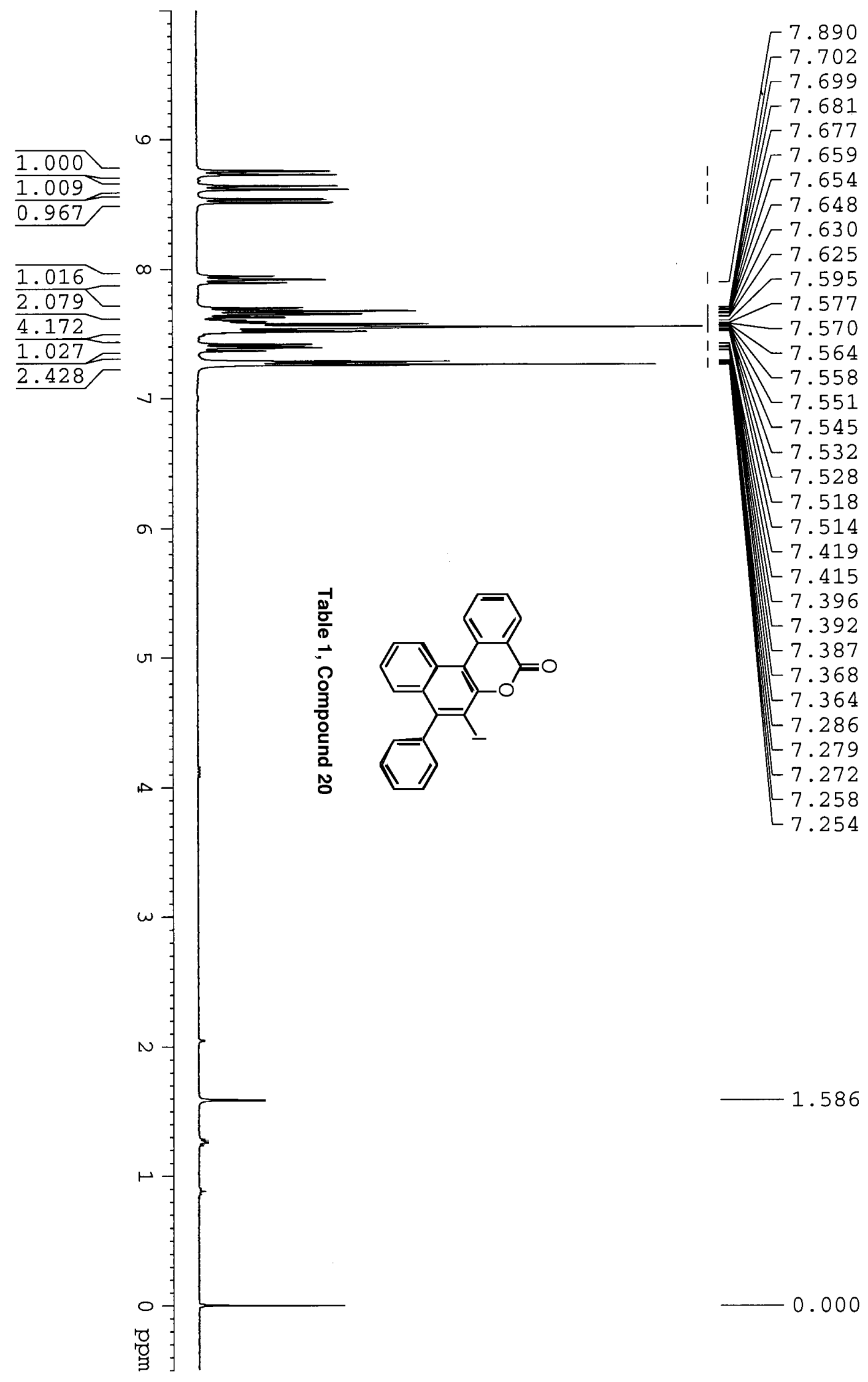




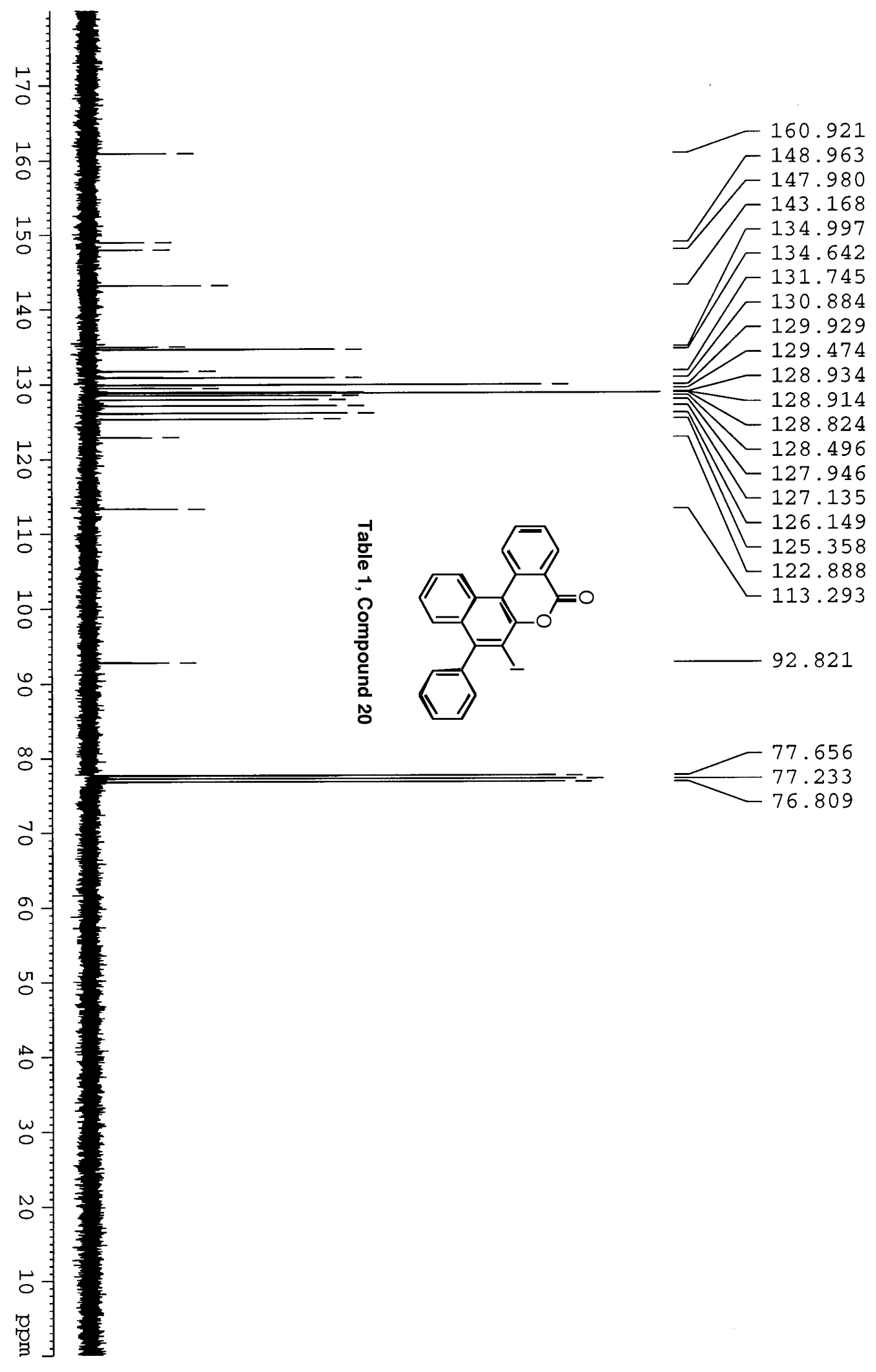




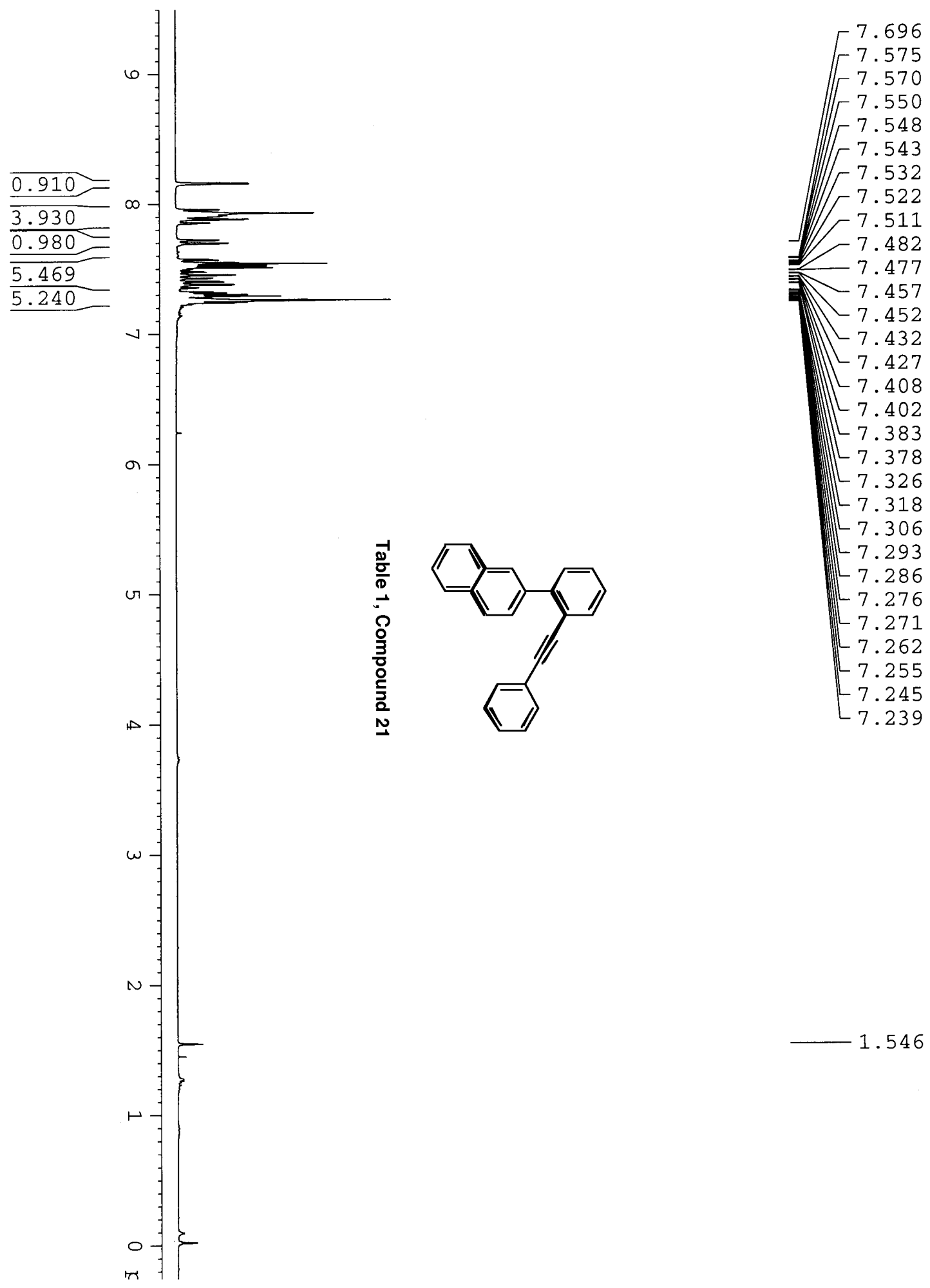




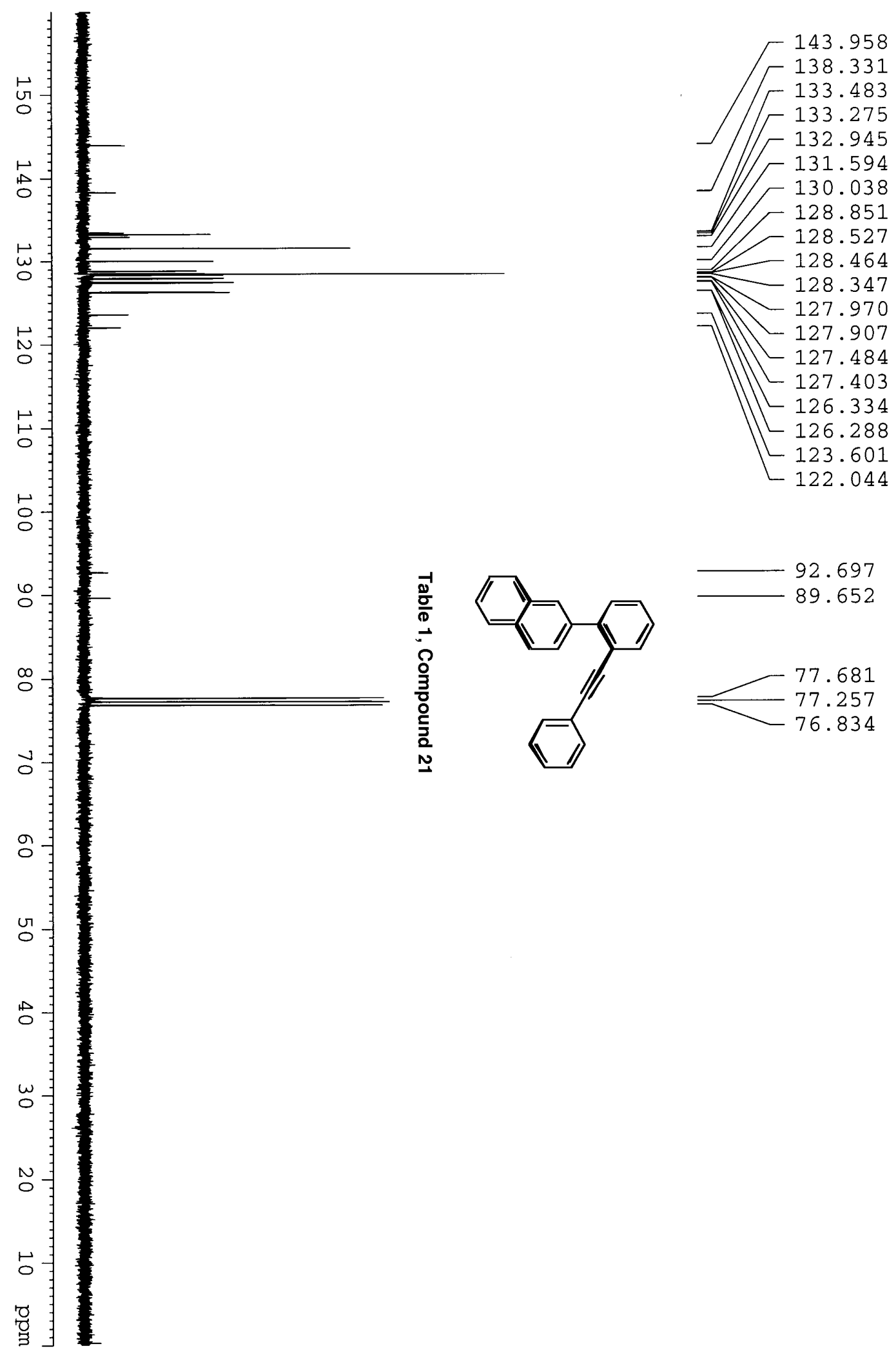



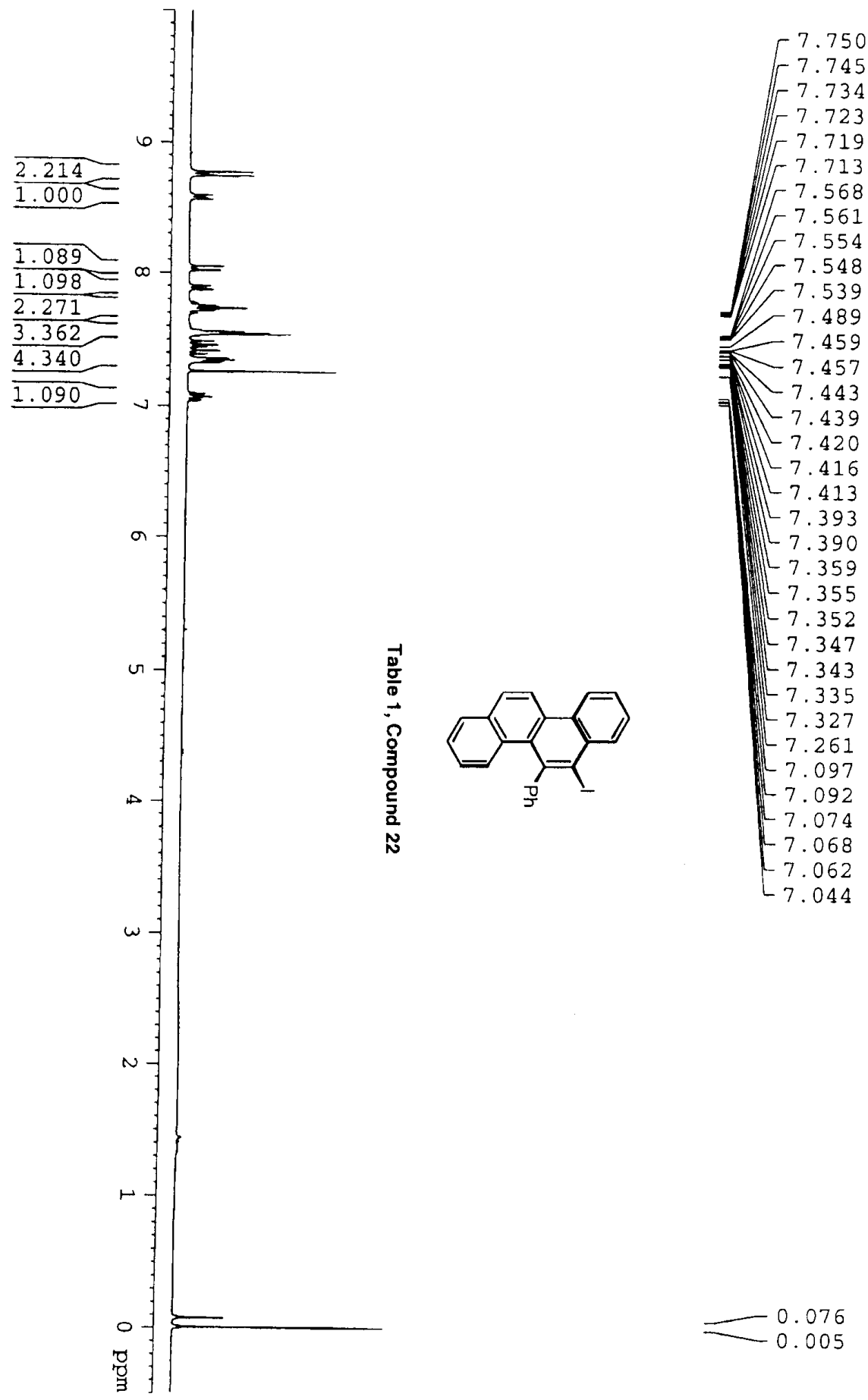

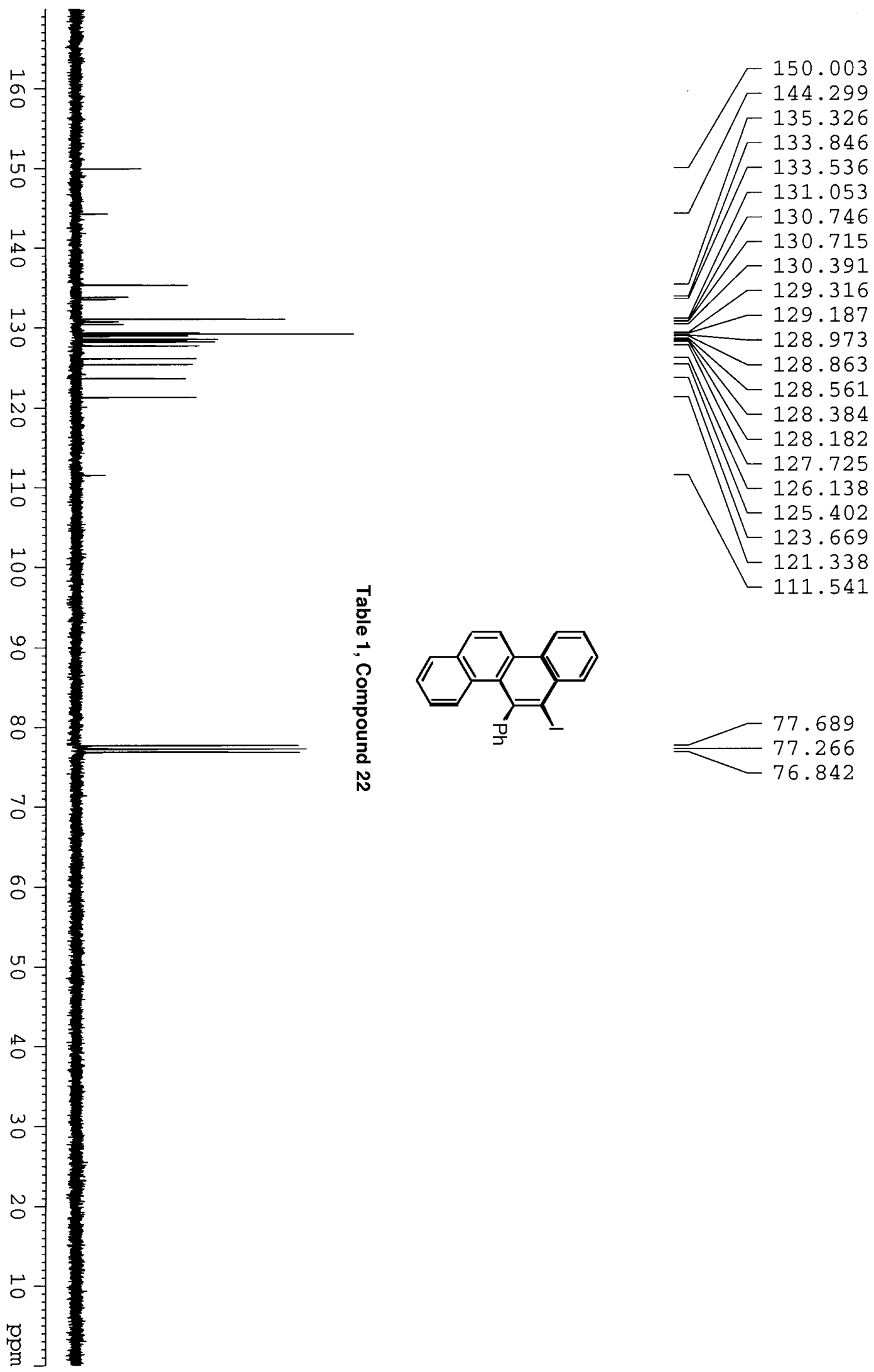

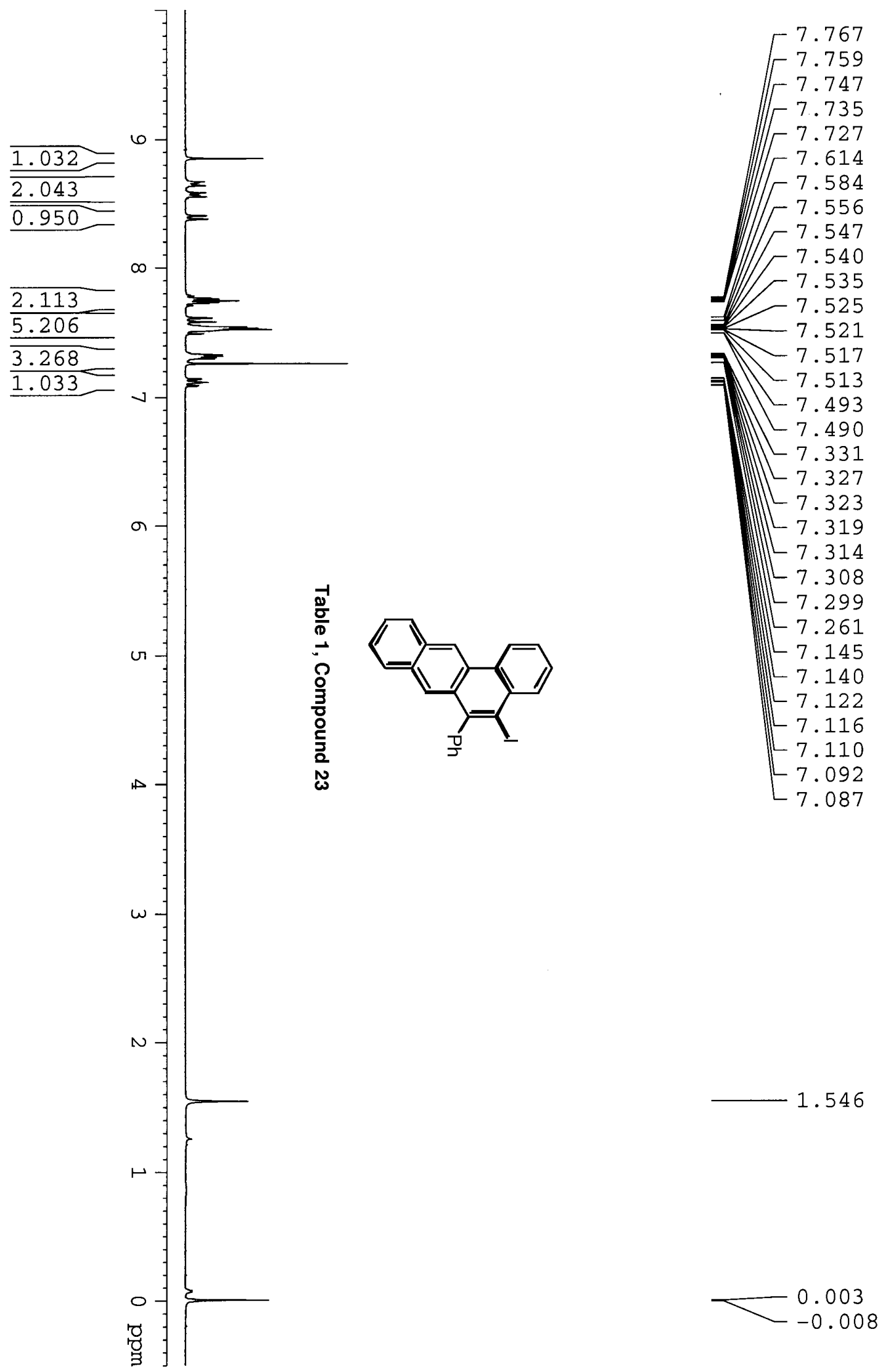


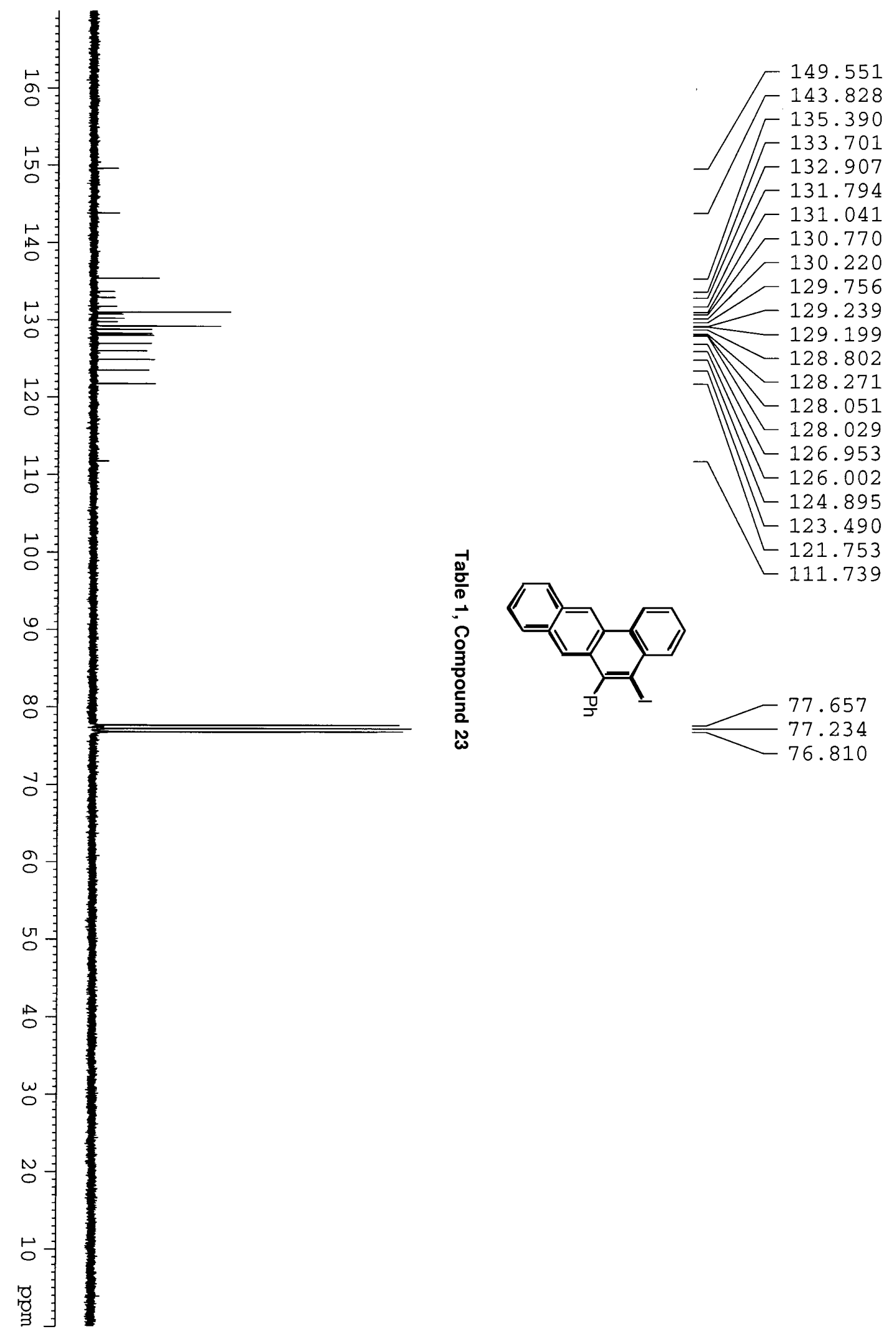



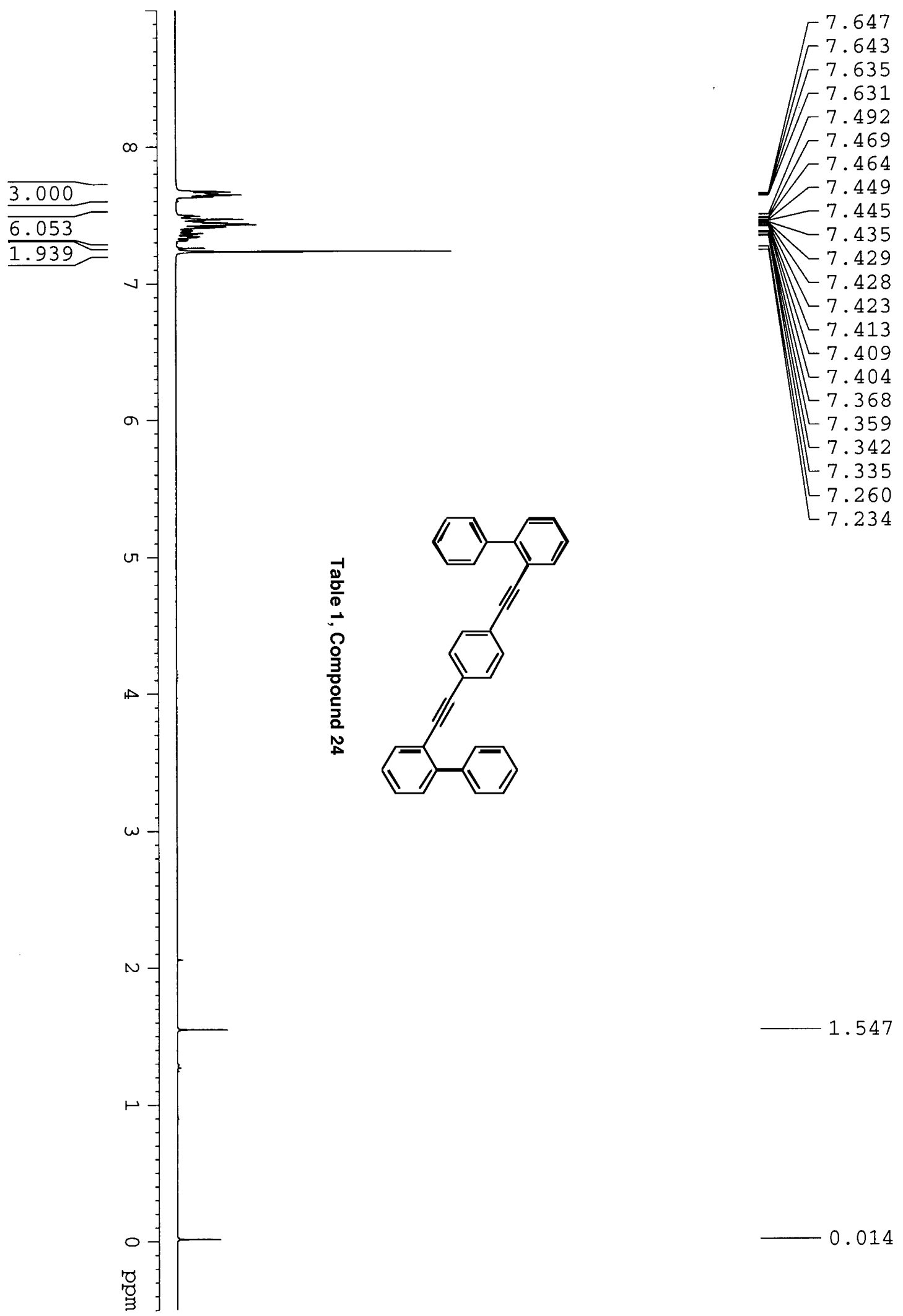

.547 0.014 


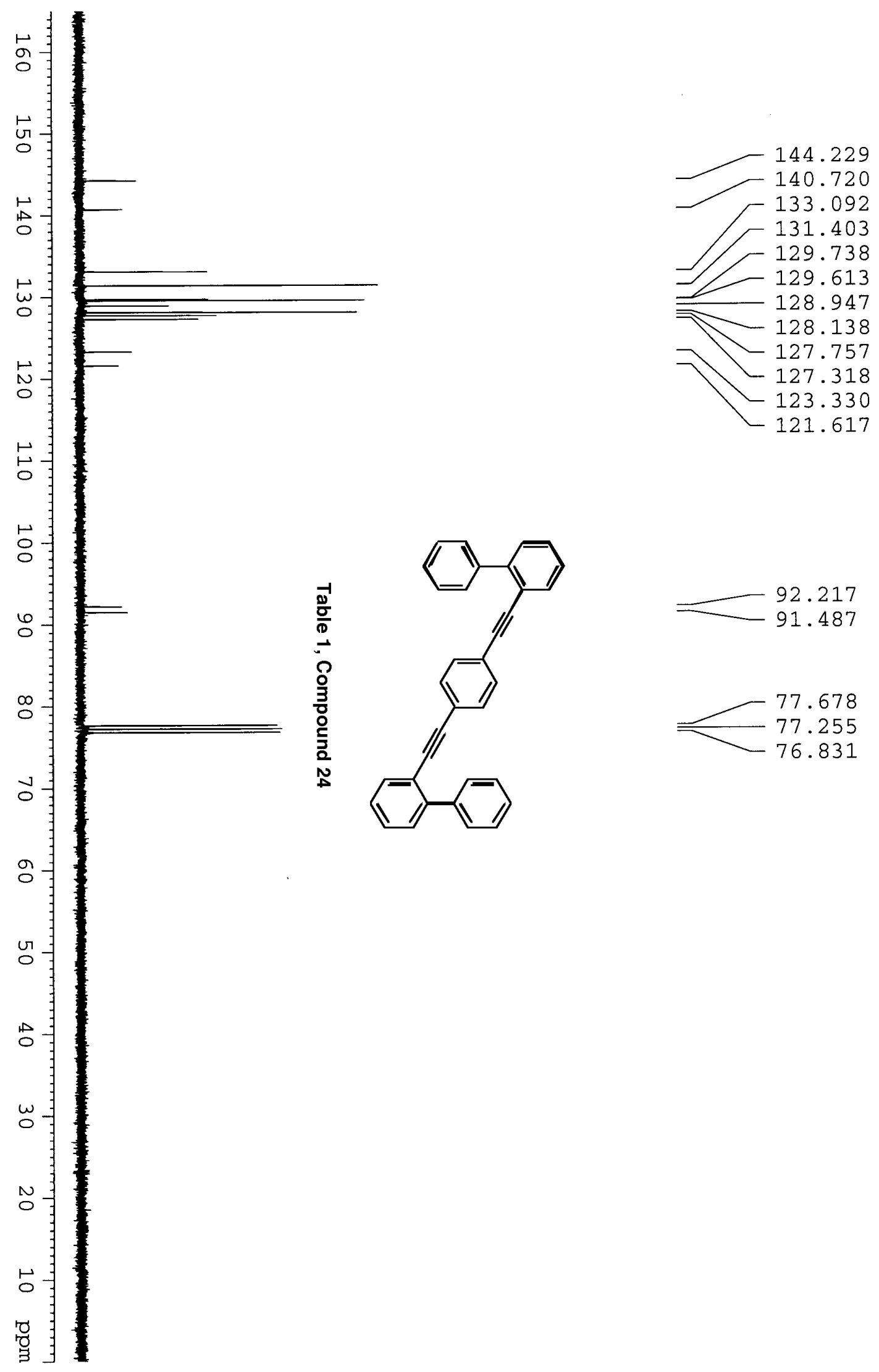




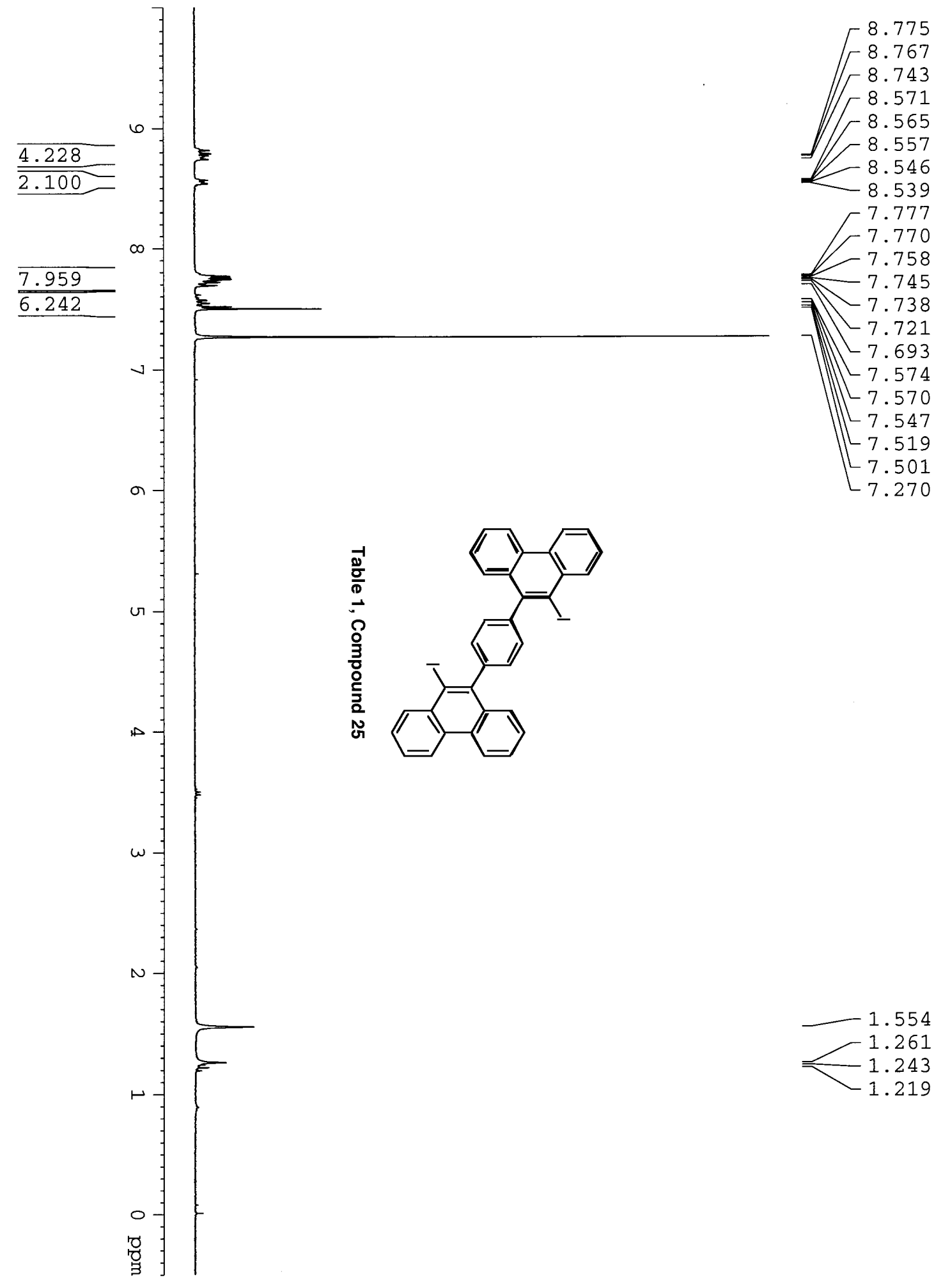



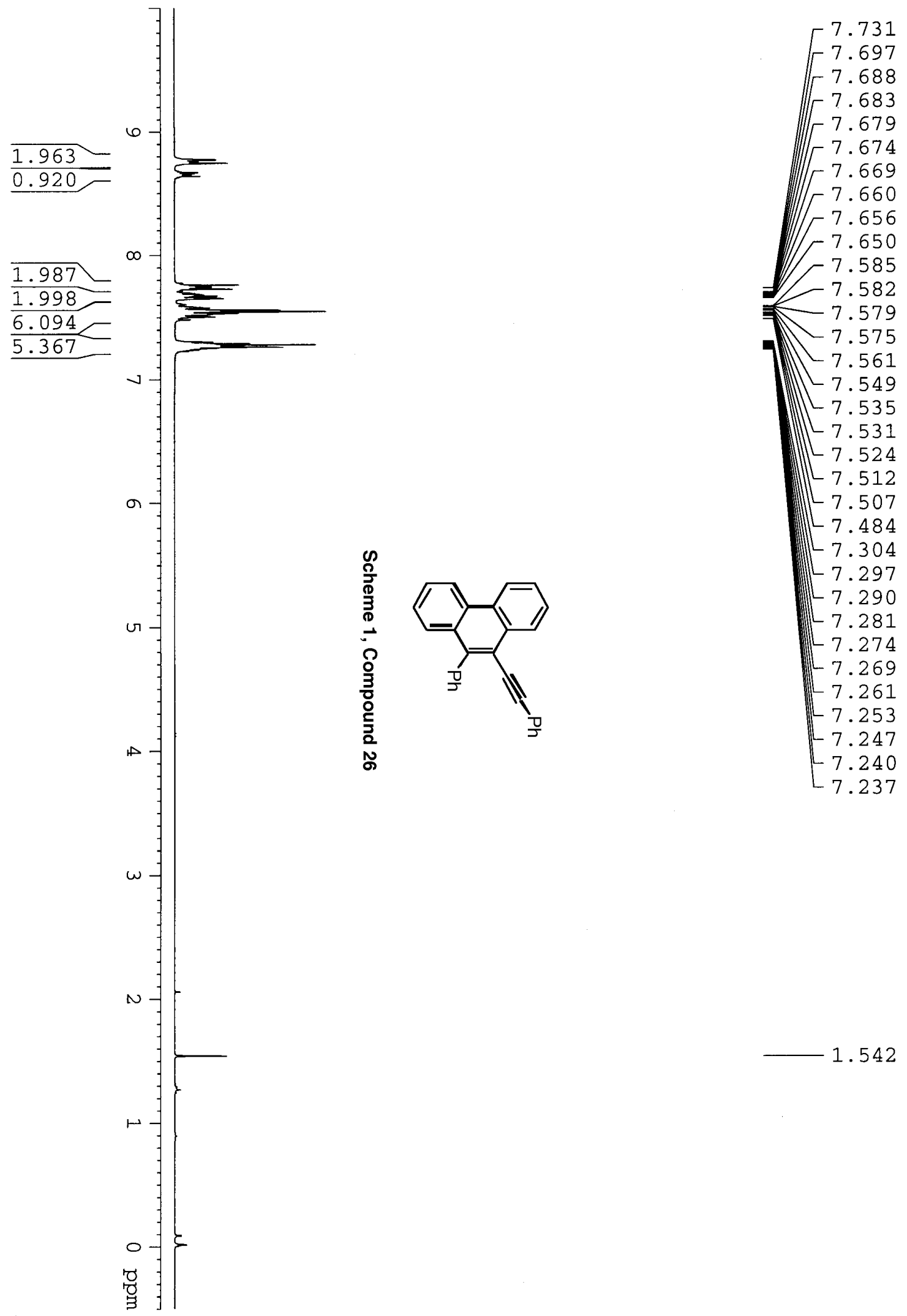

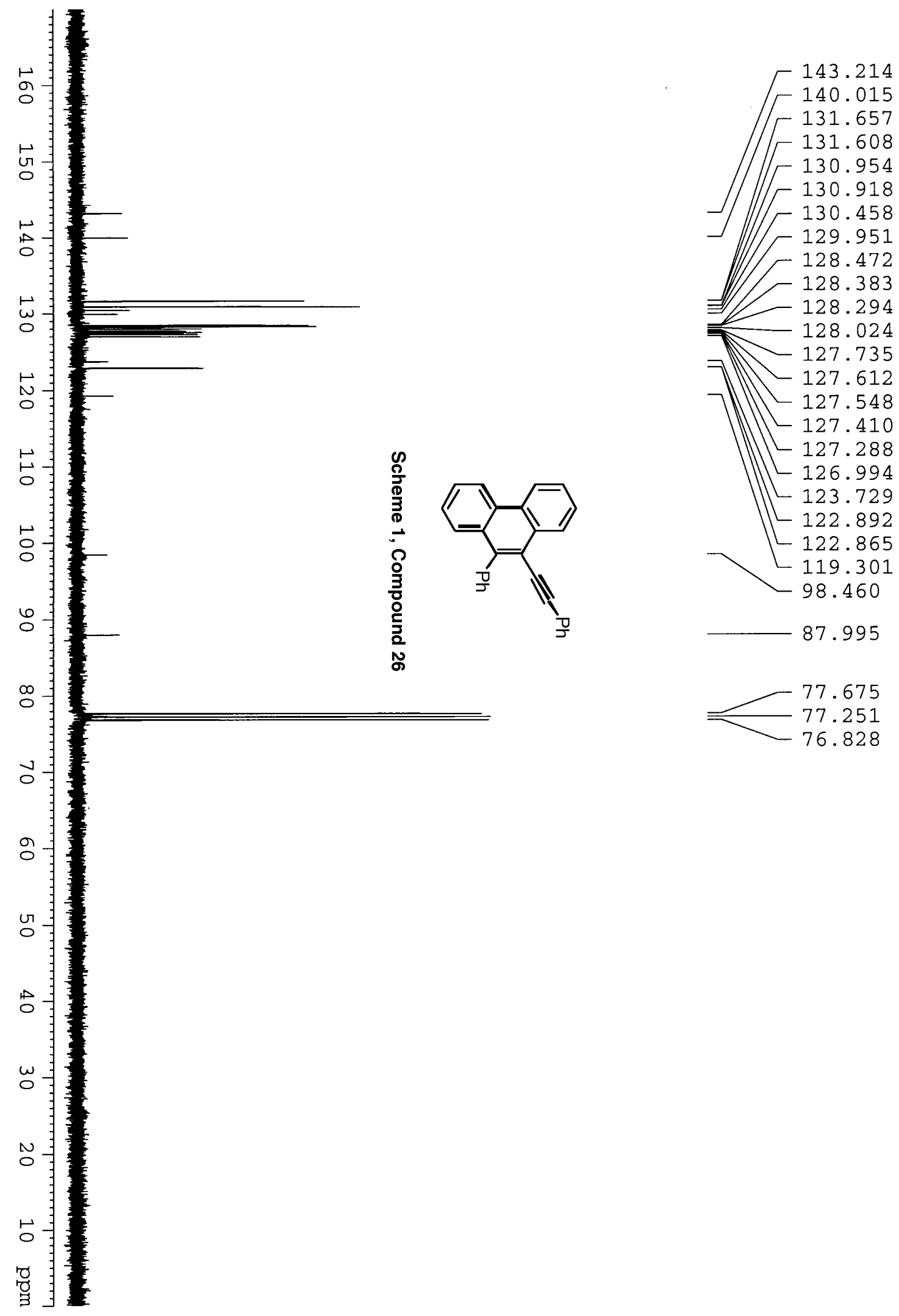

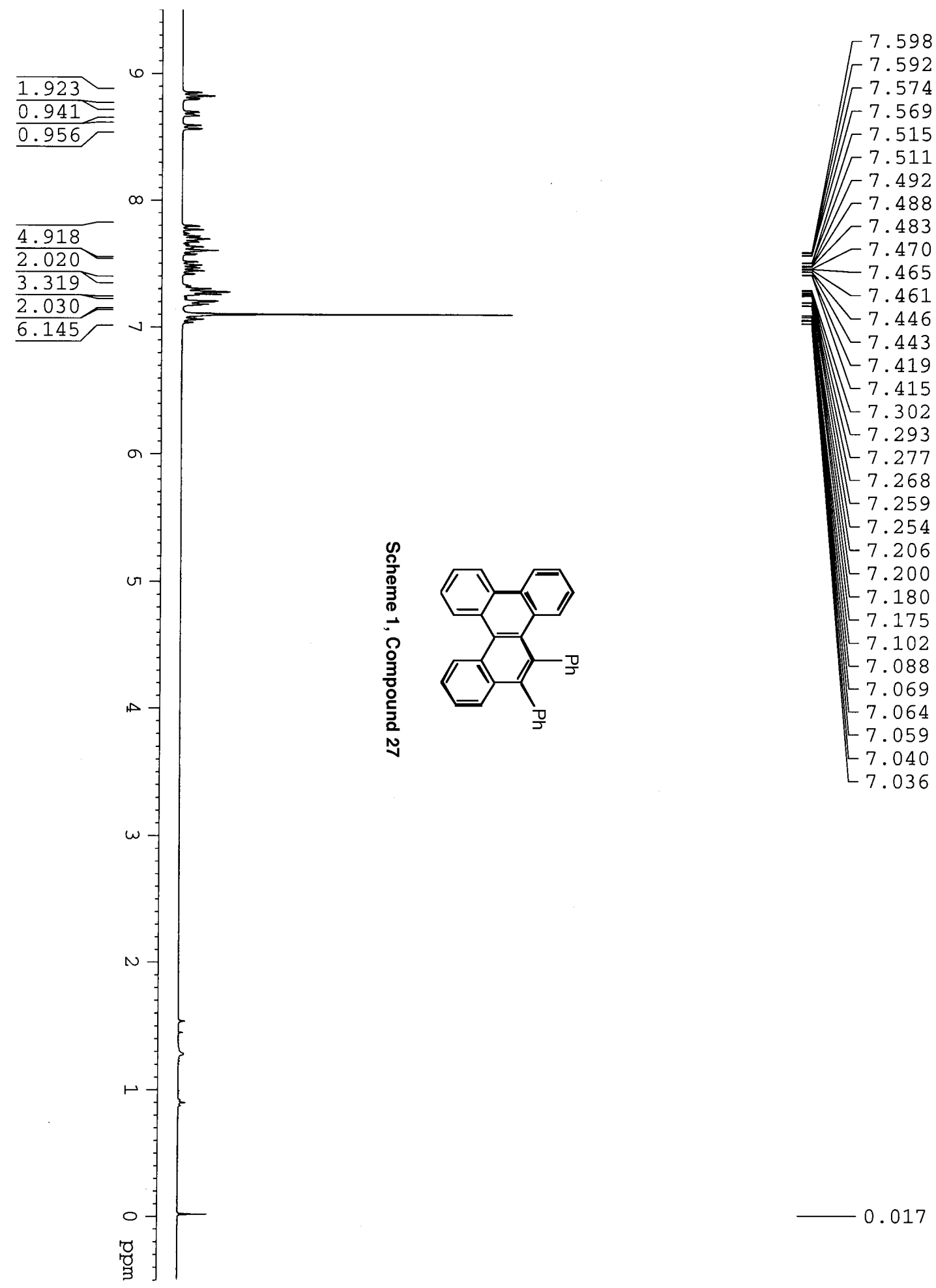


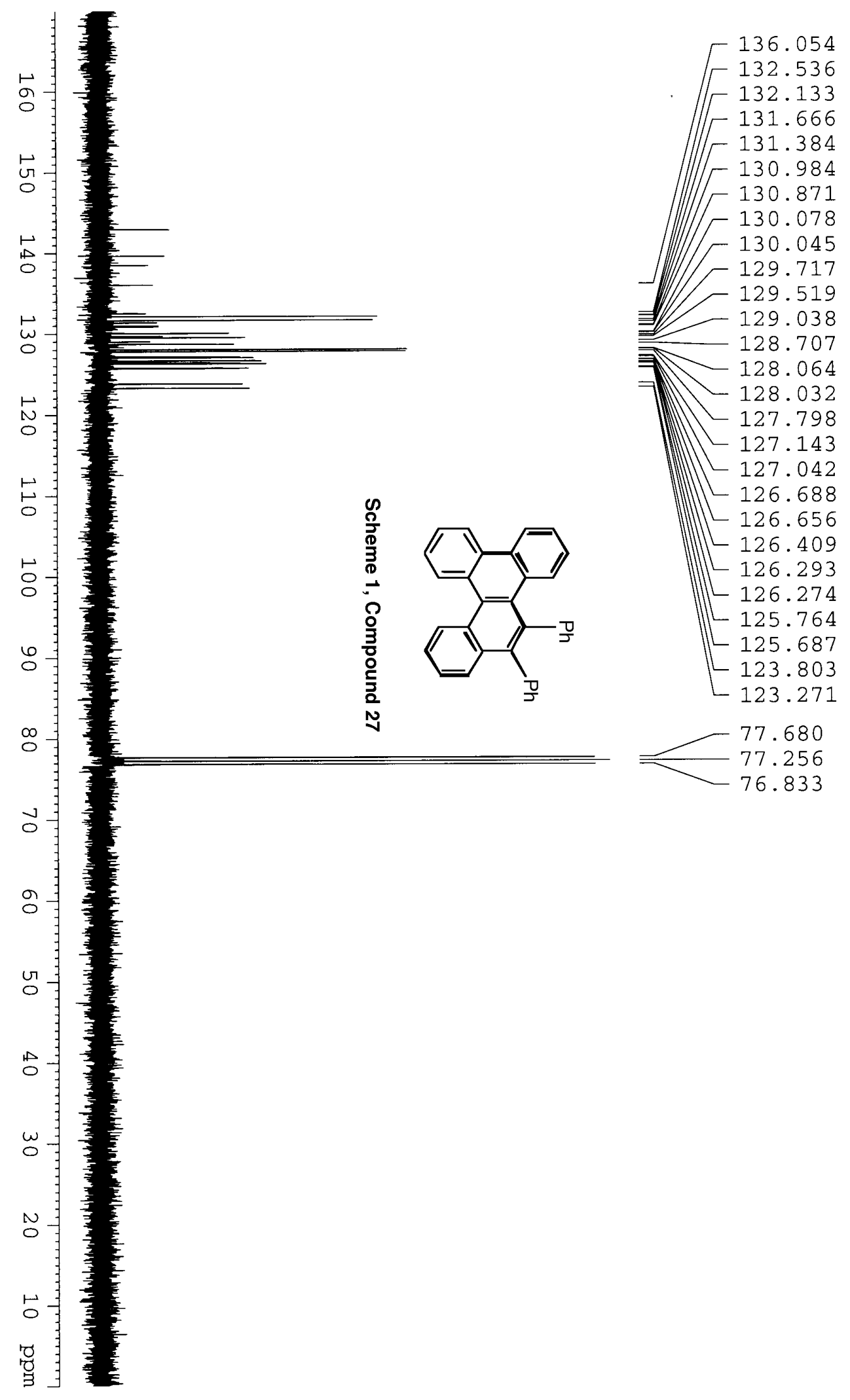

